Supporting Information

\title{
Design of Organocatalysts for Asymmetric Propargylations through Computational Screening
}

\author{
Analise C. Doney, ${ }^{\dagger}$ Benjamin J. Rooks, ${ }^{\#}$ Tongxiang Lu, and Steven E. Wheeler ${ }^{*}$ \\ Department of Chemistry, Texas A\&M University, College Station, TX 77842 \\ *E-mail: wheeler@chem.tamu.edu
}

\section{Contents}

Conformations of Catalysts Built on Scaffold 2

Page

Table S1. Analysis of errors in predicted ee's from Rooks et al.

Table S2. Predicted ee's based on enthalpies, RRHO free energies, and quasi-RRHO

free energies

Table S3. Predicted ee's for $\mathrm{R}=$ Me for selected catalysts

Absolute and relative energies, enthalpies, and free energies for all computed TS structures, along with the corresponding predicted $e e$ 's

Optimized Cartesian coordinates for all computed TS structures

\footnotetext{
${ }^{\dagger}$ Present Address: UT Southwestern Medical Center, Dallas, Texas

\# Present Address: Department of Biostatistics, University of Michigan, Ann Arbor, Michigan

${ }^{\ddagger}$ Present Address: Universal Display Corporation, Ewing, New Jersey
} 


\section{Conformations of Catalysts Built on Scaffold 2}

For scaffold 2, there are three unique conformations ( $\mathrm{i}$ - iii). Amongg these, ii and iii are $\mathrm{C}_{2}$ symmetric, whereas $\mathrm{i}$ is non-symmetric. Consequently, for conformers ii and iii we computed the 10 TS structures arising from BP1-BP5; for i we computed the 20 potential TS structures arising from BP1-BP10. All of these TS structures were included in the Boltzmann weighting. For the $\mathrm{R}=$ Me examples of catalysts built on scaffold 2, we only optimized TS structures that were reasonably low in energy based on the $\mathrm{R}=\mathrm{H}$ data.

i.<smiles>[R]c1cc(-c2ccccc2)c([X])[n+]([O-])c1-[n+]1c([X])c(-c2ccccc2)cc([R])c1[O-]</smiles>

ii.<smiles></smiles>

iii.<smiles>[R]c1cc(-c2ccccc2)c([X])[n+]([O-])c1-c1c([R])cc(-c2ccccc2)c([X])[n+]1[O-]</smiles> 
Table S1. Boltzmann-weighted relative energies derived from the data of Rooks et al., free energy differences derived from experimental $e e$ values, and the corresponding errors (in $\mathrm{kcal} / \mathrm{mol}$ ). The mean signed deviation (MSD) and mean absolute deviation (MAD) are provided.

\begin{tabular}{cccc}
\hline \hline & Theor. & Exp & \\
Cat. & $\Delta \Delta \mathrm{E}$ & $\Delta \Delta \mathrm{G}$ & Error \\
\hline \hline $1 \mathrm{a}$ & 0.75 & 0.74 & 0.02 \\
$1 \mathrm{~b}$ & 1.10 & 0.70 & 0.40 \\
$2 \mathrm{a}$ & 1.29 & 1.07 & 0.22 \\
$3 \mathrm{a}$ & 1.07 & 0.68 & 0.39 \\
$3 \mathrm{~b}$ & 1.40 & 0.86 & 0.54 \\
$4 \mathrm{a}$ & 1.07 & 0.72 & 0.35 \\
$4 \mathrm{~b}$ & 0.41 & 0.48 & -0.07 \\
$4 \mathrm{c}$ & 1.02 & 0.46 & 0.56 \\
$4 \mathrm{~d}$ & 0.53 & 0.46 & 0.07 \\
$4 \mathrm{e}$ & 0.59 & 0.37 & 0.22 \\
$5 \mathrm{a}$ & 0.77 & 0.85 & -0.08 \\
$5 \mathrm{~b}$ & 0.87 & 0.55 & 0.32 \\
$5 \mathrm{c}$ & 0.81 & 0.42 & 0.39 \\
6 & 1.38 & 1.11 & 0.28 \\
$7 \mathrm{a}$ & 0.36 & 0.39 & -0.03 \\
$7 \mathrm{~b}$ & 0.57 & 0.59 & -0.01 \\
$7 \mathrm{c}$ & 0.63 & 0.22 & 0.41 \\
8 & 0.26 & 0.41 & -0.16 \\
\hline & & $\mathrm{MSD}^{\mathrm{a}}$ & 0.21 \\
& & $\mathrm{MAD}^{\mathrm{a}}$ & 0.25 \\
\hline \hline
\end{tabular}


Table S2. Predicted $e e$ 's based on relative energies $(\mathrm{E})$, enthalpies $(\mathrm{H})$, RRHO free energies $(\mathrm{G})$, and quasi-RRHO free energies (quasi-RRHO G).

\begin{tabular}{rrrrrrrrrrrrrr}
\hline \hline $\mathbf{E}$ & $\mathbf{1}$ & $\mathbf{2}$ & $\mathbf{3}$ & $\mathbf{4}$ & $\mathbf{5}$ & $\mathbf{6}$ & $\mathbf{G}$ & $\mathbf{1}$ & $\mathbf{2}$ & $\mathbf{3}$ & $\mathbf{4}$ & $\mathbf{5}$ & $\mathbf{6}$ \\
$\mathbf{a}$ & 76 & 83 & 54 & 24 & 78 & 73 & $\mathbf{a}$ & 39 & 63 & -30 & 31 & 46 & 85 \\
$\mathbf{b}$ & 89 & 94 & 97 & 71 & 90 & 89 & $\mathbf{b}$ & 71 & 89 & 94 & 79 & 78 & 93 \\
$\mathbf{c}$ & 91 & 96 & 97 & 75 & 92 & 91 & $\mathbf{c}$ & 60 & 95 & 90 & 71 & 89 & 94 \\
$\mathbf{d}$ & 65 & 77 & 83 & -45 & 79 & 58 & $\mathbf{d}$ & 54 & 94 & 77 & 8 & 42 & 81 \\
$\mathbf{e}$ & 94 & 99 & 97 & 18 & 97 & 96 & $\mathbf{e}$ & 84 & 97 & 92 & -33 & 97 & 96 \\
$\mathbf{f}$ & 71 & -10 & 90 & -28 & 86 & 63 & $\mathbf{f}$ & 54 & -6 & 83 & 24 & 78 & 92 \\
$\mathbf{g}$ & 25 & -18 & - & 75 & 47 & -31 & $\mathbf{g}$ & 95 & 65 & - & 27 & 51 & 61 \\
$\mathbf{h}$ & 86 & 97 & 97 & 52 & 92 & 91 & $\mathbf{h}$ & 48 & 97 & 90 & 32 & 81 & 90 \\
$\mathbf{i}$ & 93 & 99 & 99 & 88 & 96 & 94 & $\mathbf{i}$ & 79 & 98 & 84 & 78 & 94 & 76 \\
$\mathbf{j}$ & 41 & 91 & 30 & 87 & 45 & 88 & $\mathbf{j}$ & 36 & -5 & 62 & 40 & 62 & 63 \\
\hline \hline $\mathbf{H}$ & \multicolumn{1}{c}{} & & & & $\mathbf{Q u a s i}-\mathbf{R R H O}$ & $\mathbf{G}$ & & & \\
\hline \hline $\mathbf{a}$ & 74 & 84 & 32 & 34 & 73 & 73 & $\mathbf{a}$ & 60 & 75 & -2 & 36 & 60 & 80 \\
$\mathbf{b}$ & 84 & 94 & 98 & 75 & 88 & 90 & $\mathbf{b}$ & 78 & 92 & 97 & 78 & 84 & 92 \\
$\mathbf{c}$ & 82 & 97 & 96 & 78 & 88 & 91 & $\mathbf{c}$ & 71 & 96 & 93 & 74 & 88 & 93 \\
$\mathbf{d}$ & 62 & 87 & 82 & -29 & 62 & 70 & $\mathbf{d}$ & 59 & 93 & 78 & -8 & 41 & 80 \\
$\mathbf{e}$ & 90 & 99 & 96 & -9 & 97 & 95 & $\mathbf{e}$ & 86 & 98 & 95 & -26 & 97 & 95 \\
$\mathbf{f}$ & 67 & -36 & 88 & -6 & 85 & 77 & $\mathbf{f}$ & 62 & -33 & 83 & 10 & 82 & 86 \\
$\mathbf{g}$ & 63 & -30 & - & 69 & 51 & -10 & $\mathbf{g}$ & 89 & 16 & - & 43 & 54 & 35 \\
$\mathbf{h}$ & 80 & 98 & 97 & 44 & 90 & 90 & $\mathbf{h}$ & 66 & 97 & 94 & 35 & 85 & 91 \\
$\mathbf{i}$ & 90 & 99 & 98 & 86 & 95 & 89 & $\mathbf{i}$ & 86 & 99 & 94 & 80 & 94 & 83 \\
$\mathbf{j}$ & 27 & 72 & -3 & 73 & 47 & 83 & $\mathbf{j}$ & 30 & 31 & 27 & 54 & 52 & 73 \\
\hline \hline & & & & & & & & & & & & &
\end{tabular}

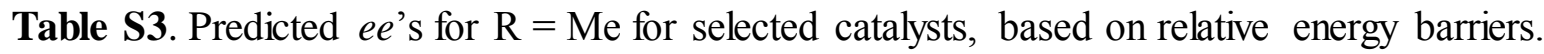

\begin{tabular}{rrrrrr}
\hline \hline & $\mathbf{1}$ & $\mathbf{2}$ & $\mathbf{3}$ & $\mathbf{4}$ & $\mathbf{5}$ \\
\hline $\mathbf{a}$ & 69 & 74 & 67 & 54 & 76 \\
$\mathbf{b}$ & & & 98 & & \\
$\mathbf{c}$ & & 95 & 97 & & \\
$\mathbf{e}$ & & 99 & 96 & & 94 \\
$\mathbf{h}$ & & 97 & 98 & & \\
$\mathbf{i}$ & & 99 & 99 & & 96 \\
\hline \hline
\end{tabular}




\section{Absolute and relative energies, enthalpies, and free energies for all computed TS structures.}

Absolute energies (E), enthalpies (H), RRHO free energies (G), and quasi-RRHO free energies (Grimme_G), in hartrees, along with the corresponding relative values in $\mathrm{kcal} / \mathrm{mol}$ and predicted ee's, for catalyst $1 \mathrm{a}(\mathrm{R}=\mathrm{Me})$

\begin{tabular}{|c|c|c|c|c|}
\hline \multicolumn{2}{|l|}{ Geometry } & \multirow{2}{*}{$\begin{array}{c}\mathrm{H} \\
70.9 \%\end{array}$} & \multirow{2}{*}{ G } & G Grimme \\
\hline $\mathrm{R}$ & $69.4 \%$ & & & $\overline{7} 8.7 \%$ \\
\hline $\mathrm{BP} 1$ & -2395.522617 & -2395.130449 & -2395.174858 & -2395.173191 \\
\hline BP2 2 & -2395.525088 & -2395.132816 & -2395.177104 & -2395.175443 \\
\hline BP3 & -2395.515102 & -2395.123008 & -2395.167542 & -2395.165834 \\
\hline BP 4 & -2395.516759 & -2395.124802 & -2395.169499 & -2395.167788 \\
\hline BP 5 & -2395.519772 & -2395.127714 & -2395.172200 & -2395.170443 \\
\hline \multicolumn{5}{|l|}{ S } \\
\hline BP1 & -2395.522039 & -2395.129973 & -2395.174416 & -2395.172668 \\
\hline BP2 & -2395.523913 & -2395.131594 & -2395.175482 & -2395.173922 \\
\hline $\mathrm{BP} 3$ & -2395.522866 & -2395.130562 & -2395.174747 & -2395.173193 \\
\hline BP 4 & -2395.517381 & -2395.125267 & -2395.169682 & -2395.167987 \\
\hline BP 5 & -2395.518860 & -2395.126623 & -2395.170917 & -2395.169320 \\
\hline \multicolumn{5}{|c|}{ Relative Energies } \\
\hline Geometry & $E$ & $\mathrm{H}(\mathrm{rel})$ & $G(r e l)$ & G_Grimme (rel) \\
\hline $\mathrm{R}$ & $69.4 \%$ & $70.9 \%$ & $81.1 \%$ & $78.7 \%$ \\
\hline BP1 & 1.6 & 1.5 & 1.4 & 1.4 \\
\hline BP2 & 0.0 & 0.0 & 0.0 & 0.0 \\
\hline BP3 & 6.3 & 6.2 & 6.0 & 6.0 \\
\hline $\mathrm{BP} 4$ & 5.2 & 5.0 & 4.8 & 4.8 \\
\hline BP 5 & 3.3 & 3.2 & 3.1 & 3.1 \\
\hline \multicolumn{5}{|l|}{$\mathrm{S}$} \\
\hline $\mathrm{BP} 1$ & 1.9 & 1.8 & 1.7 & 1.7 \\
\hline BP2 & 0.7 & 0.8 & 1.0 & 1.0 \\
\hline BP3 & 1.4 & 1.4 & 1.5 & 1.4 \\
\hline BP 4 & 4.8 & 4.7 & 4.7 & 4.7 \\
\hline BP 5 & 3.9 & 3.9 & 3.9 & 3.8 \\
\hline
\end{tabular}

Absolute energies (E), enthalpies (H), RRHO free energies (G), and quasi-RRHO free energies (Grimme_G), in hartrees, along with the corresponding relative values in $\mathrm{kcal} / \mathrm{mol}$ and predicted ee's, for catalyst la

$\begin{array}{lcccc}\text { Geometry } & \text { E } & \text { H } & \text { G } & \text { G_Grimme } \\ \text { R } & 76.0 \% & 73.7 \% & 38.6 \% & 60.3 \% \\ \text { BP1 } & -2316.912381 & -2316.575659 & -2316.616862 & -2316.615457 \\ \text { BP2 } & -2316.914381 & -2316.577682 & -2316.618689 & -2316.617390 \\ \text { BP3 } & -2316.904230 & -2316.567769 & -2316.609436 & -2316.607861 \\ \text { BP4 } & -2316.906256 & -2316.569874 & -2316.611575 & -2316.609988 \\ \text { BP5 } & -2316.909203 & -2316.572764 & -2316.614330 & -2316.612757 \\ \text { S } & & & & \\ \text { BP1 } & -2316.911732 & -2316.575314 & -2316.616786 & -2316.615279 \\ \text { BP2 } & -2316.912879 & -2316.576335 & -2316.618137 & -2316.616431 \\ \text { BP3 } & -2316.912396 & -2316.575344 & -2316.615912 & -2316.614779 \\ \text { BP4 } & -2316.906953 & -2316.570546 & -2316.612184 & -2316.610629 \\ \text { BP5 } & -2316.908377 & -2316.571808 & -2316.613188 & -2316.611705\end{array}$

Relative Energies

$\begin{array}{lcc}\text { Geometry } & E & H(r e 1) \\ \text { R } & 76.0 \% & 73.7 \% \\ \text { BP1 } & 1.3 & 1.3\end{array}$

$\mathrm{G}(\mathrm{rel})$
$38.6 \%$
1.1
G_Grimme (rel) $60.3 \%$ 1.2 


$\begin{array}{lllll}\text { BP2 } & 0.0 & 0.0 & 0.0 & 0.0 \\ \text { BP3 } & 6.4 & 6.2 & 5.8 & 6.0 \\ \text { BP4 } & 5.1 & 4.9 & 4.5 & 4.6 \\ \text { BP5 } & 3.2 & 3.1 & 2.7 & 2.9 \\ \text { S } & & & & \\ \text { BP1 } & 1.7 & 1.5 & 1.2 & 1.3 \\ \text { BP2 } & 0.9 & 0.8 & 0.3 & 1.6 \\ \text { BP3 } & 1.2 & 1.5 & 1.7 & 4.2 \\ \text { BP4 } & 4.7 & 4.5 & 4.1 & 3.6 \\ \text { BP5 } & 3.8 & 3.7 & 3.5 & \end{array}$

Absolute energies (E), enthalpies (H), RRHO free energies (G), and quasi-RRHO free energies (Grimme_G), in hartrees, along with the corresponding relative values in $\mathrm{kcal} / \mathrm{mol}$ and predicted ee's, for catalyst $1 \mathrm{~b}$

$\begin{array}{lcccc}\text { Geometry } & \text { E } & \text { H } & \text { G } & \text { G Grimme } \\ \text { R } & 88.5 \% & 83.9 \% & 70.7 \% & \overline{7} \text {. } \\ \text { BP1 } & -2515.357447 & -2515.036499 & -2515.079633 & -2515.077974 \\ \text { BP2 } & -2515.359823 & -2515.038562 & -2515.080993 & -2515.079668 \\ \text { BP3 } & -2515.348637 & -2515.027863 & -2515.071132 & -2515.069478 \\ \text { BP4 } & -2515.350969 & -2515.030139 & -2515.073486 & -2515.071782 \\ \text { BP5 } & -2515.354236 & -2515.033576 & -2515.077206 & -2515.075331 \\ \text { S } & & & & \\ \text { BP1 } & -2515.357072 & -2515.036282 & -2515.079589 & -2515.077849 \\ \text { BP2 } & -2515.357552 & -2515.036464 & -2515.079192 & -2515.077730 \\ \text { BP3 } & -2515.357560 & -2515.036441 & -2515.078906 & -2515.077569 \\ \text { BP4 } & -2515.351052 & -2515.030145 & -2515.073294 & -2515.071676 \\ \text { BP5 } & -2515.352552 & -2515.031624 & -2515.074426 & -2515.072956\end{array}$

$\begin{array}{lcccc}\text { Relative Energies } & & & \\ \text { Geometry } & E & \text { H }(r e l) & G(r e l) & \text { G_Grimme }(r e l) \\ \text { R } & 88.5 \% & 83.9 \% & 70.7 \% & 78.3 \% \\ \text { BP1 } & 1.5 & 1.3 & 0.9 & 1.1 \\ \text { BP2 } & 0.0 & 0.0 & 0.0 & 0.0 \\ \text { BP3 } & 7.0 & 6.7 & 6.2 & 6.4 \\ \text { BP4 } & 5.6 & 5.3 & 4.7 & 4.9 \\ \text { BP5 } & 3.5 & 3.1 & 2.4 & 2.7 \\ \text { S } & & & & \\ \text { BP1 } & 1.7 & 1.4 & 0.9 & 1.1 \\ \text { BP2 } & 1.4 & 1.3 & 1.1 & 1.2 \\ \text { BP3 } & 1.4 & 5.3 & 1.3 & 5.0 \\ \text { BP4 } & 5.5 & 4.4 & 4.8 & 4.2 \\ \text { BP5 } & 4.6 & & 4.1 & \end{array}$

Absolute energies (E), enthalpies (H), RRHO free energies (G), and quasi-RRHO free energies (Grimme_G), in hartrees, along with the corresponding relative values in $\mathrm{kcal} / \mathrm{mol}$ and predicted ee's, for catalyst $1 \mathrm{c}$

$\begin{array}{lcccc}\text { Geometry } & \mathrm{E} & \mathrm{H} & \mathrm{G} & \mathrm{G} \text { Grimme } \\ \text { R } & 91.3 \% & 82.3 \% & 59.6 \% & \overline{70.8 \%} \\ \text { BP1 } & -3236.155902 & -3235.837571 & -3235.881710 & -3235.879984 \\ \text { BP2 } & -3236.158054 & -3235.839260 & -3235.882898 & -3235.881355 \\ \text { BP3 } & -3236.146781 & -3235.828649 & -3235.872942 & -3235.871152 \\ \text { BP4 } & -3236.148995 & -3235.830743 & -3235.875070 & -3235.873326 \\ \text { BP5 } & -3236.152358 & -3235.833963 & -3235.878085 & -3235.876376 \\ \text { S } & & & & \\ \text { BP1 } & -3236.155435 & -3235.837139 & -3235.881552 & -3235.879699\end{array}$




\begin{tabular}{|c|c|c|c|c|}
\hline BP2 & -3236.155670 & -3235.837435 & -3235.881766 & -3235.879945 \\
\hline BP3 & -3236.155301 & -3235.836810 & -3235.880383 & -3235.878912 \\
\hline BP 4 & -3236.149771 & -3235.831528 & -3235.875877 & -3235.874109 \\
\hline BP 5 & -3236.146244 & -3235.828321 & -3235.872902 & -3235.871093 \\
\hline \multicolumn{5}{|c|}{ Relative Energies } \\
\hline Geometry & $E$ & $\mathrm{H}(r \in l)$ & $G(r e l)$ & G_Grimme(rel) \\
\hline $\mathrm{R}$ & $91.3 \%$ & $82.3 \%$ & $59.6 \%$ & $70.8 \%$ \\
\hline $\mathrm{BP} 1$ & 1.4 & 1.1 & 0.7 & 0.9 \\
\hline BP2 & 0.0 & 0.0 & 0.0 & 0.0 \\
\hline $\mathrm{BP} 3$ & 7.1 & 6.7 & 6.2 & 6.4 \\
\hline BP 4 & 5.7 & 5.3 & 4.9 & 5.0 \\
\hline BP 5 & 3.6 & 3.3 & 3.0 & 3.1 \\
\hline \multicolumn{5}{|l|}{ S } \\
\hline $\mathrm{BP} 1$ & 1.6 & 1.3 & 0.8 & 1.0 \\
\hline BP2 & 1.5 & 1.1 & 0.7 & 0.9 \\
\hline BP3 & 1.7 & 1.5 & 1.6 & 1.5 \\
\hline BP 4 & 5.2 & 4.9 & 4.4 & 4.5 \\
\hline BP 5 & 7.4 & 6.9 & 6.3 & 6.4 \\
\hline
\end{tabular}

Absolute energies (E), enthalpies (H), RRHO free energies (G), and quasi-RRHO free energies (Grimme_G), in hartrees, along with the corresponding relative values in $\mathrm{kcal} / \mathrm{mol}$ and predicted ee's, for catalyst $1 \mathrm{~d}$

\begin{tabular}{|c|c|c|c|c|}
\hline Geometry & $\mathrm{E}$ & $\mathrm{H}$ & G & G_Grimme \\
\hline $\mathrm{R}$ & $64.6 \%$ & $62.2 \%$ & $54.4 \%$ & $\overline{5} 8.7 \%$ \\
\hline BP1 & -2395.533666 & -2395.141711 & -2395.185844 & -2395.184128 \\
\hline BP2 & -2395.535138 & -2395.142923 & -2395.186220 & -2395.184991 \\
\hline $\mathrm{BP} 3$ & -2395.524392 & -2395.132649 & -2395.176644 & -2395.175042 \\
\hline BP 4 & -2395.526957 & -2395.134881 & -2395.178691 & -2395.177194 \\
\hline BP 5 & -2395.530101 & -2395.137915 & -2395.181606 & -2395.180187 \\
\hline$S$ & & & & \\
\hline $\mathrm{BP} 1$ & -2395.532585 & -2395.140679 & -2395.184832 & -2395.183189 \\
\hline BP2 & -2395.534018 & -2395.141877 & -2395.185481 & -2395.184059 \\
\hline $\mathrm{BP} 3$ & -2395.533358 & -2395.141137 & -2395.184339 & -2395.183131 \\
\hline BP 4 & -2395.528812 & -2395.136900 & -2395.180702 & -2395.179278 \\
\hline BP 5 & -2395.523147 & -2395.130955 & -2395.174627 & -2395.173141 \\
\hline Relative & Energies & & & \\
\hline Geometry & $E$ & $\mathrm{H}(r e l)$ & G(rel) & G_Grimme (rel) \\
\hline $\mathrm{R}$ & $64.6 \%$ & $62.2 \%$ & $54.4 \%$ & $58.7 \%$ \\
\hline BP1 & 0.9 & 0.8 & 0.2 & 0.5 \\
\hline BP2 & 0.0 & 0.0 & 0.0 & 0.0 \\
\hline BP3 & 6.7 & 6.4 & 6.0 & 6.2 \\
\hline BP 4 & 5.1 & 5.0 & 4.7 & 4.9 \\
\hline BP 5 & 3.2 & 3.1 & 2.9 & 3.0 \\
\hline S & & & & \\
\hline BP 1 & 1.6 & 1.4 & 0.9 & 1.1 \\
\hline BP 2 & 0.7 & 0.7 & 0.5 & 0.6 \\
\hline BP3 & 1.1 & 1.1 & 1.2 & 1.2 \\
\hline BP 4 & 4.0 & 3.8 & 3.5 & 3.6 \\
\hline BP 5 & 7.5 & 7.5 & 7.3 & 7.4 \\
\hline
\end{tabular}

Absolute energies (E), enthalpies (H), RRHO free energies (G), and quasi-RRHO free energies (Grimme_G), in hartrees, along with the corresponding relative values in $\mathrm{kcal} / \mathrm{mol}$ and predicted ee's, for catalyst $1 \mathrm{e}$ 


\begin{tabular}{|c|c|c|c|c|}
\hline Geometry & $\mathrm{E}$ & $\mathrm{H}$ & G & G_Grimme \\
\hline$R$ & $94.1 \%$ & $90.3 \%$ & $84.0 \%$ & $\overline{8} 6.2 \%$ \\
\hline BP1 & -2990.904043 & -2990.556398 & -2990.605597 & -2990.603157 \\
\hline BP2 & -2990.906059 & -2990.558219 & -2990.606985 & -2990.604713 \\
\hline BP3 & -2990.893697 & -2990.546593 & -2990.596028 & -2990.593534 \\
\hline BP 4 & -2990.897548 & -2990.549820 & -2990.598300 & -2990.596314 \\
\hline BP 5 & -2990.896288 & -2990.549078 & -2990.598613 & -2990.596085 \\
\hline S & & & & \\
\hline $\mathrm{BP} 1$ & -2990.903676 & -2990.556218 & -2990.605387 & -2990.602980 \\
\hline BP2 & -2990.903204 & -2990.555538 & -2990.604531 & -2990.602209 \\
\hline BP3 & -2990.901671 & -2990.554173 & -2990.603249 & -2990.600906 \\
\hline BP 4 & -2990.898294 & -2990.550689 & -2990.599810 & -2990.597497 \\
\hline BP 5 & -2990.891197 & -2990.543743 & -2990.594011 & -2990.591151 \\
\hline Relative & Energies & & & \\
\hline Geometry & $\mathrm{E}$ & $\mathrm{H}(r e l)$ & $G(r e l)$ & G Grimme(rel) \\
\hline $\mathrm{R}$ & $94.1 \%$ & $90.3 \%$ & $84.0 \%$ & $86.2 \%$ \\
\hline BP1 & 1.3 & 1.1 & 0.9 & 1.0 \\
\hline BP2 & 0.0 & 0.0 & 0.0 & 0.0 \\
\hline BP3 & 7.8 & 7.3 & 6.9 & 7.0 \\
\hline BP 4 & 5.3 & 5.3 & 5.4 & 5.3 \\
\hline BP 5 & 6.1 & 5.7 & 5.3 & 5.4 \\
\hline S & & & & \\
\hline BP1 & 1.5 & 1.3 & 1.0 & 1.1 \\
\hline BP2 & 1.8 & 1.7 & 1.5 & 1.6 \\
\hline BP3 & 2.8 & 2.5 & 2.3 & 2.4 \\
\hline BP 4 & 4.9 & 4.7 & 4.5 & 4.5 \\
\hline BP 5 & 9.3 & 9.1 & 8.1 & 8.5 \\
\hline
\end{tabular}

Absolute energies (E), enthalpies (H), RRHO free energies (G), and quasi-RRHO free energies (Grimme_G), in hartrees, along with the corresponding relative values in kcal/mol and predicted ee's, for catalyst if

$\begin{array}{lcccc}\text { Geometry } & \text { E } & \text { H } & \text { G } & \text { G_Grimme } \\ \text { R } & 70.6 \% & 66.8 \% & 54.2 \% & 61.9 \% \\ \text { BP1 } & -2552.734916 & -2552.229117 & -2552.277958 & -2552.275943 \\ \text { BP2 } & -2552.736966 & -2552.231257 & -2552.280274 & -2552.278193 \\ \text { BP3 } & -2552.726274 & -2552.220852 & -2552.270595 & -2552.268236 \\ \text { BP4 } & -2552.729064 & -2552.223303 & -2552.272867 & -2552.270534 \\ \text { BP5 } & -2552.733478 & -2552.227823 & -2552.276828 & -2552.274768 \\ \text { S } & & & & \\ \text { BP1 } & -2552.733343 & -2552.227658 & -2552.277037 & -2552.274741 \\ \text { BP2 } & -2552.735529 & -2552.230052 & -2552.279458 & -2552.277181 \\ \text { BP3 } & -2552.735398 & -2552.229527 & -2552.278168 & -2552.276274 \\ \text { BP4 } & -2552.730380 & -2552.224477 & -2552.273313 & -2552.271344 \\ \text { BP5 } & -2552.723323 & -2552.217762 & -2552.267020 & -2552.264859\end{array}$

Relative Energies

$\begin{array}{lc}\text { Geometry } & E \\ \text { R } & 70.6 \% \\ \text { BP1 } & 1.3 \\ \text { BP2 } & 0.0 \\ \text { BP3 } & 6.7 \\ \text { BP4 } & 5.0 \\ \text { BP5 } & 2.2 \\ \text { S } & \end{array}$

$\begin{array}{clc}H(r e l) & \text { G }(r e l) & \text { G_Grimme }(r e 1) \\ 66.8 \% & 54.2 \% & 61.9 \% \\ 1.3 & 1.5 & 1.4 \\ 0.0 & 0.0 & 0.0 \\ 6.5 & 6.1 & 6.2 \\ 5.0 & 4.6 & 4.8 \\ 2.2 & 2.2 & 2.1 \\ 2.3 & 2.0 & 2.2\end{array}$




$\begin{array}{lllll}\text { BP2 } & 0.9 & 0.8 & 0.5 & 0.6 \\ \text { BP3 } & 1.0 & 1.1 & 1.3 & 1.2 \\ \text { BP4 } & 4.1 & 4.3 & 4.4 & 4.3 \\ \text { BP5 } & 8.6 & 8.5 & 8.3 & 8.4\end{array}$

Absolute energies (E), enthalpies (H), RRHO free energies (G), and quasi-RRHO free energies (Grimme G), in hartrees, along with the corresponding relative values in $\mathrm{kcal} / \mathrm{mol}$ and predicted ee's, for catalyst $1 \mathrm{~g}$

$\begin{array}{lcccc}\text { Geometry } & \text { E } & \text { H } & \text { G } & \text { G Grimme } \\ \text { R } & 25.2 \% & 62.8 \% & 95.2 \% & 88.8 \% \\ \text { BP1 } & -2631.309895 & -2630.748956 & -2630.800346 & -2630.797895 \\ \text { BP2 } & -2631.309451 & -2630.747751 & -2630.797914 & -2630.795909 \\ \text { BP3 } & -2631.297463 & -2630.736344 & -2630.787218 & -2630.784848 \\ \text { BP4 } & -2631.302707 & -2630.740647 & -2630.790222 & -2630.788436 \\ \text { BP5 } & -2631.305041 & -2630.743589 & -2630.793673 & -2630.791676 \\ \text { S } & & & & \\ \text { BP1 } & -2631.307951 & -2630.746293 & -2630.796900 & -2630.794706 \\ \text { BP2 } & -2631.309789 & -2630.748091 & -2630.797969 & -2630.796110 \\ \text { BP3 } & -2631.306192 & -2630.744777 & -2630.795176 & -2630.793175 \\ \text { BP4 } & -2631.305591 & -2630.743928 & -2630.793857 & -2630.791921 \\ \text { BP5 } & -2631.294843 & -2630.734023 & -2630.784725 & -2630.782469\end{array}$

Relative Energies

\begin{tabular}{|c|c|c|c|c|}
\hline Geometry & $\mathrm{E}$ & $\mathrm{H}(r e l)$ & $G(r e l)$ & G_Grimme (rel) \\
\hline $\mathrm{R}$ & $25.2 \%$ & $62.8 \%$ & $95.2 \%$ & $88.8 \%$ \\
\hline BP1 & 0.0 & 0.0 & 0.0 & 0.0 \\
\hline BP2 & 0.3 & 0.8 & 1.5 & 1.2 \\
\hline BP3 & 7.8 & 7.9 & 8.2 & 8.2 \\
\hline BP 4 & 4.5 & 5.2 & 6.4 & 5.9 \\
\hline BP5 & 3.0 & 3.4 & 4.2 & 3.9 \\
\hline \multicolumn{5}{|l|}{$\mathrm{S}$} \\
\hline BP1 & 1.2 & 1.7 & 2.2 & 2.0 \\
\hline BP2 & 0.1 & 0.5 & 1.5 & 1.1 \\
\hline BP3 & 2.3 & 2.6 & 3.2 & 3.0 \\
\hline BP 4 & 2.7 & 3.2 & 4.1 & 3.7 \\
\hline BP5 & 9.4 & 9.4 & 9.8 & 9.7 \\
\hline
\end{tabular}

Absolute energies (E), enthalpies (H), RRHO free energies (G), and quasi-RRHO free energies (Grimme_G), in hartrees, along with the corresponding relative values in $\mathrm{kcal} / \mathrm{mol}$ and predicted ee's, for catalyst $1 \mathrm{~h}$

$\begin{array}{lcccc}\text { Geometry } & \text { E } & \text { H } & \text { G } & \text { G_Grimme } \\ \text { R } & 86.3 \% & 80.0 \% & 48.1 \% & 65.9 \% \\ \text { BP1 } & -2469.128687 & -2468.773843 & -2468.819428 & -2468.817654 \\ \text { BP2 } & -2469.130537 & -2468.775381 & -2468.820512 & -2468.818902 \\ \text { BP3 } & -2469.119366 & -2468.764857 & -2468.811163 & -2468.809128 \\ \text { BP4 } & -2469.123088 & -2468.768288 & -2468.813902 & -2468.812148 \\ \text { BP5 } & -2469.125369 & -2468.770327 & -2468.815113 & -2468.813670 \\ \text { S } & & & & \\ \text { BP1 } & -2469.128758 & -2468.773860 & -2468.819728 & -2468.817774 \\ \text { BP2 } & -2469.127914 & -2468.773153 & -2468.819205 & -2468.817190 \\ \text { BP3 } & -2469.127476 & -2468.772405 & -2468.817618 & -2468.815985 \\ \text { BP4 } & -2469.121989 & -2468.767040 & -2468.812470 & -2468.810785 \\ \text { BP5 } & -2469.119997 & -2468.765220 & -2468.810560 & -2468.808932\end{array}$

Relative Energies 


\begin{tabular}{|c|c|c|c|c|}
\hline Geometry & $\mathrm{E}$ & $H(r e l)$ & $G(r e l)$ & G_Grimme (rel) \\
\hline $\mathrm{R}$ & $86.3 \%$ & $80.0 \%$ & $48.1 \%$ & $65.9 \%$ \\
\hline BP1 & 1.2 & 1.0 & 0.7 & 0.8 \\
\hline BP2 & 0.0 & 0.0 & 0.0 & 0.0 \\
\hline BP3 & 7.0 & 6.6 & 5.9 & 6.1 \\
\hline BP 4 & 4.7 & 4.5 & 4.1 & 4.2 \\
\hline BP5 & 3.2 & 3.2 & 3.4 & 3.3 \\
\hline \multicolumn{5}{|l|}{$\mathrm{S}$} \\
\hline BP1 & 1.1 & 1.0 & 0.5 & 0.7 \\
\hline BP2 & 1.6 & 1.4 & 0.8 & 1.1 \\
\hline BP3 & 1.9 & 1.9 & 1.8 & 1.8 \\
\hline BP 4 & 5.4 & 5.2 & 5.0 & 5.1 \\
\hline BP5 & 6.6 & 6.4 & 6.2 & 6.3 \\
\hline
\end{tabular}

Absolute energies (E), enthalpies (H), RRHO free energies (G), and quasi-RRHO free energies (Grimme_G), in hartrees, along with the corresponding relative values in $\mathrm{kcal} / \mathrm{mol}$ and predicted ee's, for catalyst $1 \mathrm{i}$

\begin{tabular}{|c|c|c|c|c|}
\hline Geometry & $\mathrm{E}$ & $\mathrm{H}$ & G & G Grimme \\
\hline $\mathrm{R}$ & $92.5 \%$ & $90.2 \%$ & $79.0 \%$ & $\overline{8} 6.0 \%$ \\
\hline BP1 & -2501.298489 & -2500.964305 & -2501.009673 & -2501.007878 \\
\hline BP2 & -2501.300739 & -2500.966244 & -2501.011182 & -2501.009581 \\
\hline BP3 & -2501.289200 & -2500.954820 & -2501.000209 & -2500.998390 \\
\hline BP 4 & -2501.290216 & -2500.956216 & -2501.002158 & -2501.000204 \\
\hline BP5 & -2501.295209 & -2500.960896 & -2501.006158 & -2501.004395 \\
\hline $\mathrm{S}$ & & & & \\
\hline BP1 & -2501.298552 & -2500.964234 & -2501.009638 & -2501.007784 \\
\hline BP2 & -2501.297756 & -2500.963539 & -2501.009250 & -2501.007273 \\
\hline BP3 & -2501.297173 & -2500.962595 & -2501.007154 & -2501.005701 \\
\hline BP 4 & -2501.291003 & -2500.956762 & -2501.002397 & -2501.000543 \\
\hline BP5 & -2501.290936 & -2500.956752 & -2501.002294 & -2501.000463 \\
\hline Relative & Energies & & & \\
\hline Geometry & $\mathrm{E}$ & $H(r e l)$ & $G(r e l)$ & G Grimme (rel) \\
\hline $\mathrm{R}$ & $92.5 \%$ & $90.2 \%$ & $79.0 \%$ & $86.0 \%$ \\
\hline BP1 & 1.4 & 1.2 & 0.9 & 1.1 \\
\hline BP2 & 0.0 & 0.0 & 0.0 & 0.0 \\
\hline BP3 & 7.2 & 7.2 & 6.9 & 7.0 \\
\hline BP 4 & 6.6 & 6.3 & 5.7 & 5.9 \\
\hline BP5 & 3.5 & 3.4 & 3.2 & 3.3 \\
\hline S & & & & \\
\hline BP1 & 1.4 & 1.3 & 1.0 & 1.1 \\
\hline BP2 & 1.9 & 1.7 & 1.2 & 1.4 \\
\hline BP3 & 2.2 & 2.3 & 2.5 & 2.4 \\
\hline BP 4 & 6.1 & 6.0 & 5.5 & 5.7 \\
\hline$B$ & 6.2 & 6.0 & 5.6 & 5.7 \\
\hline
\end{tabular}

Absolute energies (E), enthalpies (H), RRHO free energies (G), and quasi-RRHO free energies (Grimme_G), in hartrees, along with the corresponding relative values in $\mathrm{kcal} / \mathrm{mol}$ and predicted ee's, for catalyst $1 j$

$\begin{array}{lcccc}\text { Geometry } & \text { E } & \text { H } & \text { G } & \text { G Grimme } \\ \text { R } & 40.8 \% & 26.8 \% & 36.1 \% & \overline{3} 0.2 \% \\ \text { BP1 } & -2778.827646 & -2778.329537 & -2778.381277 & -2778.378225 \\ \text { BP2 } & -2778.829297 & -2778.331078 & -2778.382361 & -2778.379525 \\ \text { BP3 } & -2778.817140 & -2778.318899 & -2778.370081 & -2778.367374 \\ \text { BP4 } & -2778.811866 & -2778.313668 & -2778.365649 & -2778.362524\end{array}$




$\begin{array}{lcccc}\text { BP5 } & -2778.822835 & -2778.324569 & -2778.375287 & -2778.372683 \\ \text { S } & & & & \\ \text { BP1 } & -2778.828786 & -2778.330775 & -2778.381984 & -2778.379201 \\ \text { BP2 } & -2778.825659 & -2778.327652 & -2778.378991 & -2778.376156 \\ \text { BP3 } & -2778.826428 & -2778.328120 & -2778.378926 & -2778.376279 \\ \text { BP4 } & -2778.819180 & -2778.320890 & -2778.371824 & -2778.369221 \\ \text { BP5 } & -2778.819595 & -2778.321489 & -2778.372543 & -2778.369903 \\ \text { Relative Energies } & & & \\ \text { Geometry } & \text { E } & \text { H rel) } & \text { G(rel) } & \text { G_Grimme (rel) } \\ \text { R } & 40.8 \% & 26.8 \% & 36.1 \% & 30.2 \% \\ \text { BP1 } & 1.0 & 1.0 & 0.7 & 0.8 \\ \text { BP2 } & 0.0 & 0.0 & 0.0 & 0 \\ \text { BP3 } & 7.6 & 7.6 & 7.7 & 10.7 \\ \text { BP4 } & 10.9 & 10.9 & 10.5 & 4.3 \\ \text { BP5 } & 4.1 & 4.1 & 4.4 & 0.2 \\ \text { S } & 0.3 & 0.2 & 0.2 & 2.1 \\ \text { BP1 } & 2.3 & 2.1 & 2.1 & 2.0 \\ \text { BP2 } & 1.8 & 1.9 & 2.2 & 6.5 \\ \text { BP3 } & 6.3 & 6.4 & 6.6 & 6.0 \\ \text { BP4 } & 6.1 & 6.0 & 6.2 & 0 \\ \text { BP5 } & & & & 0\end{array}$

Absolute energies (E), enthalpies (H), RRHO free energies (G), and quasi-RRHO free energies (Grimme_G), in hartrees, along with the corresponding relative values in $\mathrm{kcal} / \mathrm{mol}$ and predicted ee's, for catalyst $3 a(\mathrm{R}=\mathrm{Me})$

$\begin{array}{lcccc}\text { Geometry } & \mathrm{E} & \mathrm{H} & \mathrm{G} & \mathrm{G} \\ \mathrm{R} & 67.3 \% & 69.5 \% & 79.8 \% & 77.2 \% \\ \text { BP1 } & -2474.135343 & -2473.687886 & -2473.736797 & -2473.734327 \\ \text { BP2 } & -2474.139213 & -2473.691517 & -2473.739662 & -2473.737607 \\ \text { BP3 } & -2474.128236 & -2473.681075 & -2473.730288 & -2473.727816 \\ \text { BP4 } & -2474.129741 & -2473.682394 & -2473.731474 & -2473.729025 \\ \text { BP5 } & -2474.132780 & -2473.685320 & -2473.733922 & -2473.731652 \\ \text { S } & & & & \\ \text { BP1 } & -2474.134752 & -2473.687425 & -2473.736468 & -2473.734000 \\ \text { BP2 } & -2474.138150 & -2473.690396 & -2473.738196 & -2473.736245 \\ \text { BP3 } & -2474.136680 & -2473.688968 & -2473.737042 & -2473.735062 \\ \text { BP4 } & -2474.130258 & -2473.682991 & -2473.731659 & -2473.729348 \\ \text { BP5 } & -2474.131892 & -2473.684411 & -2473.732807 & -2473.730603\end{array}$

Relative Energies

$\begin{array}{lcccc}\text { Geometry } & E & \text { H (rel) } & \text { G(rel) } & \text { G_Grimme (rel) } \\ \text { R } & 67.3 \% & 69.5 \% & 79.8 \% & 77.2 \% \\ \text { BP1 } & 2.4 & 2.3 & 1.8 & 2.1 \\ \text { BP2 } & 0.0 & 0.0 & 0.0 & 0.0 \\ \text { BP3 } & 6.9 & 6.6 & 5.9 & 6.1 \\ \text { BP4 } & 5.9 & 5.7 & 5.1 & 5.4 \\ \text { BP5 } & 4.0 & 3.9 & 3.6 & 3.7 \\ \text { S } & & & & 2.3 \\ \text { BP1 } & 2.8 & 2.6 & 2.0 & 0.9 \\ \text { BP2 } & 0.7 & 0.7 & 0.9 & 1.6 \\ \text { BP3 } & 1.6 & 1.6 & 1.6 & 5.2 \\ \text { BP4 } & 5.6 & 5.4 & 5.0 & 4.4 \\ \text { BP5 } & 4.6 & 4.5 & 4.3 & \end{array}$




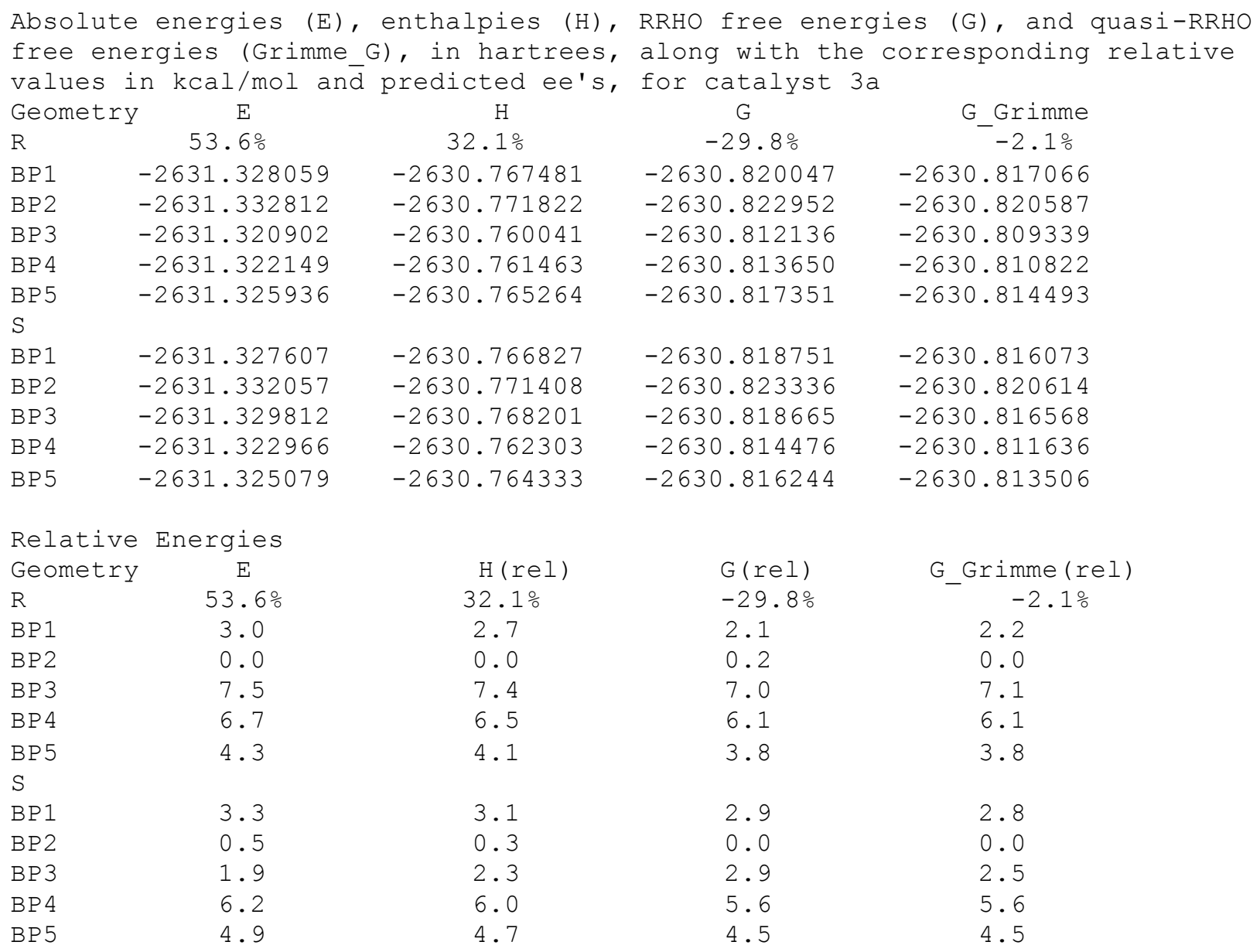

Absolute energies (E), enthalpies (H), RRHO free energies (G), and quasi-RRHO free energies (Grimme G), in hartrees, along with the corresponding relative values in $\mathrm{kcal} / \mathrm{mol}$ and predicted ee's, for catalyst 3b $(\mathrm{R}=\mathrm{Me})$

$\begin{array}{lcccc}\text { Geometry } & \text { E } & \text { H } & \text { G } & \text { G_Grimme } \\ \text { R } & 98.4 \% & 97.4 \% & 89.8 \% & 94.0 \% \\ \text { BP1 } & -2908.381355 & -2907.779908 & -2907.835664 & -2907.832848 \\ \text { BP2 } & -2908.386343 & -2907.784904 & -2907.839836 & -2907.837584 \\ \text { BP3 } & -2908.373860 & -2907.772569 & -2907.828708 & -2907.825928 \\ \text { BP4 } & -2908.374717 & -2907.773503 & -2907.829656 & -2907.826841 \\ \text { BP5 } & -2908.378757 & -2907.777852 & -2907.834732 & -2907.831552 \\ \text { S } & & & & \\ \text { BP1 } & -2908.381190 & -2907.779628 & -2907.835072 & -2907.832394 \\ \text { BP2 } & -2908.383189 & -2907.782135 & -2907.838000 & -2907.835399 \\ \text { BP3 } & -2908.382361 & -2907.780902 & -2907.836135 & -2907.833679 \\ \text { BP4 } & -2908.374758 & -2907.773807 & -2907.830324 & -2907.827345 \\ \text { BP5 } & -2908.376439 & -2907.775083 & -2907.831148 & -2907.828401\end{array}$

Relative Energies

$\begin{array}{lcccc}\text { Geometry } & E & \text { H }(r e l) & \text { G(rel) } & \text { G_Grimme }(r e l) \\ \text { R } & 98.4 \% & 97.4 \% & 89.8 \% & 94.0 \% \\ \text { BP1 } & 3.1 & 3.1 & 2.6 & 3.0 \\ \text { BP2 } & 0.0 & 0.0 & 0.0 & 0.0 \\ \text { BP3 } & 7.8 & 7.7 & 7.0 & 7.3 \\ \text { BP4 } & 7.3 & 7.2 & 6.4 & 6.7\end{array}$




$\begin{array}{lllll}\text { BP5 } & 4.8 & 4.4 & 3.2 & 3.8 \\ \text { S } & & & & \\ \text { BP1 } & 3.2 & 3.3 & 3.0 & 3.3 \\ \text { BP2 } & 2.0 & 1.7 & 1.2 & 1.4 \\ \text { BP3 } & 2.5 & 2.5 & 2.3 & 2.5 \\ \text { BP4 } & 7.3 & 7.0 & 6.0 & 6.4 \\ \text { BP5 } & 6.2 & 6.2 & 5.5 & 5.8\end{array}$

Absolute energies (E), enthalpies (H), RRHO free energies (G), and quasi-RRHO free energies (Grimme_G), in hartrees, along with the corresponding relative values in $\mathrm{kcal} / \mathrm{mol}$ and predicted ee's, for catalyst 3b

$\begin{array}{lcccc}\text { Geometry } & \text { E } & \text { H } & \text { G } & \text { G Grimme } \\ \text { R } & 97.3 \% & 97.8 \% & 94.2 \% & 96.5 \% \\ \text { BP1 } & -2829.770352 & -2829.224979 & -2829.278548 & -2829.275710 \\ \text { BP2 } & -2829.775008 & -2829.229415 & -2829.281656 & -2829.279436 \\ \text { BP3 } & -2829.762718 & -2829.216973 & -2829.269658 & -2829.267223 \\ \text { BP4 } & -2829.764119 & -2829.218734 & -2829.271956 & -2829.269260 \\ \text { BP5 } & -2829.767725 & -2829.222061 & -2829.275195 & -2829.272505 \\ \text { S } & & & & \\ \text { BP1 } & -2829.770221 & -2829.224637 & -2829.277842 & -2829.275156 \\ \text { BP2 } & -2829.772215 & -2829.226492 & -2829.279367 & -2829.276819 \\ \text { BP3 } & -2829.771242 & -2829.225562 & -2829.278104 & -2829.275695 \\ \text { BP4 } & -2829.764224 & -2829.218598 & -2829.271876 & -2829.269104 \\ \text { BP5 } & -2829.765623 & -2829.220114 & -2829.273116 & -2829.270510\end{array}$

Relative Energies

$\begin{array}{lcclc}\text { Geometry } & \text { E } & \text { H }(r e l) & \text { G }(r e l) & \text { G_Grimme }(r e l) \\ \text { R } & 97.3 \% & 97.8 \% & 94.2 \% & 96.5 \% \\ \text { BP1 } & 2.9 & 2.8 & 2.0 & 2.3 \\ \text { BP2 } & 0.0 & 0.0 & 0.0 & 0.0 \\ \text { BP3 } & 7.7 & 7.8 & 7.5 & 7.7 \\ \text { BP4 } & 6.8 & 6.7 & 6.1 & 6.4 \\ \text { BP5 } & 4.6 & 4.6 & & 4.3 \\ \text { S } & & 3.0 & 2.4 & \\ \text { BP1 } & 3.0 & 1.8 & 1.4 & 2.7 \\ \text { BP2 } & 1.8 & 2.4 & 2.2 & 2.6 \\ \text { BP3 } & 2.4 & 6.8 & 6.1 & 6.5 \\ \text { BP4 } & 6.8 & 5.8 & 5.4 & 5.6 \\ \text { BP5 } & 5.9 & & & \end{array}$

Absolute energies (E), enthalpies (H), RRHO free energies (G), and quasi-RRHO free energies (Grimme_G), in hartrees, along with the corresponding relative values in $\mathrm{kcal} / \mathrm{mol}$ and predicted ee's, for catalyst $3 \mathrm{c}(\mathrm{R}=\mathrm{Me})$

$\begin{array}{lcccc}\text { Geometry } & \mathrm{E} & \mathrm{H} & \mathrm{G} & \text { GGrimme } \\ \text { R } & 97.0 \% & 97.6 \% & 98.4 \% & 98.3 \% \\ \text { BP1 } & -3629.167194 & -3628.568912 & -3628.626789 & -3628.623619 \\ \text { BP2 } & -3629.172112 & -3628.573742 & -3628.630680 & -3628.628021 \\ \text { BP3 } & -3629.159363 & -3628.560864 & -3628.617998 & -3628.615181 \\ \text { BP4 } & -3629.160268 & -3628.561874 & -3628.619588 & -3628.616584 \\ \text { BP5 } & -3629.164396 & -3628.565852 & -3628.622418 & -3628.619747 \\ \text { S } & & & & \\ \text { BP1 } & -3629.166779 & -3628.568593 & -3628.626580 & -3628.623370 \\ \text { BP2 } & -3629.169498 & -3628.570987 & -3628.627577 & -3628.625009 \\ \text { BP3 } & -3629.167491 & -3628.568907 & -3628.625110 & -3628.622672 \\ \text { BP4 } & -3629.160670 & -3628.562322 & -3628.619617 & -3628.616656\end{array}$




\begin{tabular}{|c|c|c|c|c|}
\hline BP 5 & -3629.157200 & -3628.559084 & -3628.616702 & -3628.613676 \\
\hline \multicolumn{5}{|c|}{ Relative Energies } \\
\hline Geometry & $E$ & $\mathrm{H}(\mathrm{rel})$ & $G(r e l)$ & G_Grimme (rel) \\
\hline $\mathrm{R}$ & $97.0 \%$ & $97.6 \%$ & $98.4 \%$ & $-98.3 \%$ \\
\hline BP1 & 3.1 & 3.0 & 2.4 & 2.8 \\
\hline BP2 & 0.0 & 0.0 & 0.0 & 0.0 \\
\hline $\mathrm{BP} 3$ & 8.0 & 8.1 & 8.0 & 8.1 \\
\hline BP 4 & 7.4 & 7.4 & 7.0 & 7.2 \\
\hline BP 5 & 4.8 & 5.0 & 5.2 & 5.2 \\
\hline \multicolumn{5}{|l|}{ S } \\
\hline BP1 & 3.3 & 3.2 & 2.6 & 2.9 \\
\hline BP2 & 1.6 & 1.7 & 1.9 & 1.9 \\
\hline BP 3 & 2.9 & 3.0 & 3.5 & 3.4 \\
\hline $\mathrm{BP} 4$ & 7.2 & 7.2 & 6.9 & 7.1 \\
\hline BP 5 & 9.4 & 9.2 & 8.8 & 9.0 \\
\hline
\end{tabular}

Absolute energies (E), enthalpies (H), RRHO free energies (G), and quasi-RRHO free energies (Grimme_G), in hartrees, along with the corresponding relative values in $\mathrm{kcal} / \mathrm{mol}$ and predicted ee's, for catalyst 3c

$\begin{array}{lcccc}\text { Geometry } & \mathrm{E} & \mathrm{H} & \mathrm{G} & \mathrm{G} \text { Grimme } \\ \text { R } & 97.0 \% & 96.3 \% & 89.6 \% & 93.3 \% \\ \text { BP1 } & -3550.555500 & -3550.012789 & -3550.067511 & -3550.064493 \\ \text { BP2 } & -3550.560081 & -3550.017236 & -3550.070433 & -3550.068152 \\ \text { BP3 } & -3550.547325 & -3550.004489 & -3550.058504 & -3550.055893 \\ \text { BP4 } & -3550.548489 & -3550.005980 & -3550.060891 & -3550.057868 \\ \text { BP5 } & -3550.552554 & -3550.009517 & -3550.062944 & -3550.060487 \\ \text { S } & & & & \\ \text { BP1 } & -3550.554965 & -3550.012260 & -3550.067026 & -3550.064002 \\ \text { BP2 } & -3550.557470 & -3550.014755 & -3550.068589 & -3550.066045 \\ \text { BP3 } & -3550.555408 & -3550.012493 & -3550.065872 & -3550.063487 \\ \text { BP4 } & -3550.549276 & -3550.006347 & -3550.060903 & -3550.057977 \\ \text { BP5 } & -3550.544997 & -3550.002478 & -3550.057235 & -3550.054276\end{array}$

$\begin{array}{lcccc}\begin{array}{l}\text { Relative Energies } \\ \text { Geometry }\end{array} & \text { E } & \text { H }(r e l) & \text { G(rel) } & \text { G_Grimme }(r e l) \\ \text { R } & 97.0 \% & 96.3 \% & 89.6 \% & 93.3 \% \\ \text { BP1 } & 2.9 & 2.8 & 1.8 & 2.3 \\ \text { BP2 } & 0.0 & 0.0 & 0.0 & 0.0 \\ \text { BP3 } & 8.0 & 8.0 & 7.5 & 7.7 \\ \text { BP4 } & 7.3 & 7.1 & 6.0 & 6.5 \\ \text { BP5 } & 4.7 & 4.8 & 4.7 & 4.8 \\ \text { S } & & & & 2.6 \\ \text { BP1 } & 3.2 & 3.1 & 1.2 & 1.3 \\ \text { BP2 } & 1.6 & 1.6 & 2.9 & 2.9 \\ \text { BP3 } & 2.9 & 3.0 & 6.0 & 6.4 \\ \text { BP4 } & 6.8 & 6.8 & 8.3 & 8.7 \\ \text { BP5 } & 9.5 & 9.3 & & \end{array}$

Absolute energies (E), enthalpies (H), RRHO free energies (G), and quasi-RRHO free energies (Grimme_G), in hartrees, along with the corresponding relative values in kcal/mol and predicted ee's, for catalyst $3 \mathrm{~d}$
Geometry $\mathrm{E}$
$\mathrm{R}$
$82.9 \%$
$\mathrm{H}$
BP1
$82.0 \%$
$76.5 \%$
G Grimme
$78.2 \%$
$\begin{array}{lll}-2709.312821 & -2709.366745 & -2709.363948\end{array}$ 


\begin{tabular}{|c|c|c|c|c|}
\hline BP2 & -2709.934553 & -2709.316671 & -2709.369462 & -2709.367319 \\
\hline BP3 & -2709.922229 & -2709.304322 & -2709.358242 & -2709.355556 \\
\hline BP 4 & -2709.923835 & -2709.306098 & -2709.359417 & -2709.356949 \\
\hline BP5 & -2709.927586 & -2709.309747 & -2709.363010 & -2709.360515 \\
\hline \multicolumn{5}{|l|}{$\mathrm{S}$} \\
\hline BP1 & -2709.929823 & -2709.311537 & -2709.364539 & -2709.362147 \\
\hline BP2 & -2709.933080 & -2709.315238 & -2709.368219 & -2709.366021 \\
\hline BP3 & -2709.930436 & -2709.312267 & -2709.364926 & -2709.362823 \\
\hline BP 4 & -2709.925926 & -2709.308186 & -2709.361557 & -2709.359109 \\
\hline BP 5 & -2709.919109 & -2709.301426 & -2709.355916 & -2709.353005 \\
\hline \multicolumn{5}{|c|}{ Relative Energies } \\
\hline Geometry & $\mathrm{E}$ & $H(r e l)$ & $G(r e l)$ & G_Grimme (rel) \\
\hline $\mathrm{R}$ & $82.9 \%$ & $82.0 \%$ & $76.5 \%$ & $78.2 \%$ \\
\hline BP1 & 2.4 & 2.4 & 1.7 & 2.1 \\
\hline BP2 & 0.0 & 0.0 & 0.0 & 0.0 \\
\hline BP3 & 7.7 & 7.7 & 7.0 & 7.4 \\
\hline BP 4 & 6.7 & 6.6 & 6.3 & 6.5 \\
\hline BP5 & 4.4 & 4.3 & 4.0 & 4.3 \\
\hline \multicolumn{5}{|l|}{$\mathrm{S}$} \\
\hline BP1 & 3.0 & 3.2 & 3.1 & 3.2 \\
\hline BP2 & 0.9 & 0.9 & 0.8 & 0.8 \\
\hline BP3 & 2.6 & 2.8 & 2.8 & 2.8 \\
\hline BP 4 & 5.4 & 5.3 & 5.0 & 5.2 \\
\hline BP 5 & 9.7 & 9.6 & 8.5 & 9.0 \\
\hline
\end{tabular}

Absolute energies (E), enthalpies (H), RRHO free energies (G), and quasi-RRHO free energies (Grimme_G), in hartrees, along with the corresponding relative values in $\mathrm{kcal} / \mathrm{mol}$ and predicted ee's, for catalyst $3 e(\mathrm{R}=\mathrm{Me})$

\begin{tabular}{|c|c|c|c|c|}
\hline Geometry & $E$ & $\mathrm{H}$ & G & G Grimme \\
\hline $\mathrm{R}$ & $96.1 \%$ & $92.7 \%$ & $83.5 \%$ & $87.7 \%$ \\
\hline $\mathrm{BP} 1$ & -3383.894233 & -3383.266861 & -3383.328387 & -3383.325139 \\
\hline BP2 & -3383.898753 & -3383.271057 & -3383.331981 & -3383.329061 \\
\hline BP3 & -3383.896337 & -3383.268994 & -3383.330520 & -3383.327314 \\
\hline BP 4 & -3383.886502 & -3383.258991 & -3383.320433 & -3383.317400 \\
\hline BP 5 & -3383.886747 & -3383.259301 & -3383.320835 & -3383.317661 \\
\hline $\mathrm{S}$ & & & & \\
\hline BP1 & -3383.894205 & -3383.266973 & -3383.328940 & -3383.325572 \\
\hline BP2 & -3383.896330 & -3383.269039 & -3383.330503 & -3383.327379 \\
\hline BP3 & -3383.893360 & -3383.265208 & -3383.325738 & $-3383 \cdot 322820$ \\
\hline BP 4 & -3383.886747 & -3383.259302 & -3383.320840 & -3383.317665 \\
\hline BP 5 & -3383.886501 & -3383.258989 & -3383.320421 & -3383.317390 \\
\hline Relative & e Energies & & & \\
\hline Geometry & $\mathrm{E}$ & $\mathrm{H}(r e l)$ & $G(r e l)$ & G Grimme (rel) \\
\hline $\mathrm{R}$ & $96.1 \%$ & $92.7 \%$ & $83.5 \%$ & $87.7 \%$ \\
\hline BP1 & 2.8 & 2.6 & 2.3 & 2.5 \\
\hline BP2 & 0.0 & 0.0 & 0.0 & 0.0 \\
\hline BP 3 & 1.5 & 1.3 & 0.9 & 1.1 \\
\hline BP 4 & 7.7 & 7.6 & 7.2 & 7.3 \\
\hline BP5 & 7.5 & 7.4 & 7.0 & 7.2 \\
\hline $\mathrm{S}$ & & & & \\
\hline BP1 & 2.9 & 2.6 & 1.9 & 2.2 \\
\hline BP2 & 1.5 & 1.3 & 0.9 & 1.1 \\
\hline BP3 & 3.4 & 3.7 & 3.9 & 3.9 \\
\hline $\mathrm{BP} 4$ & 7.5 & 7.4 & 7.0 & 7.2 \\
\hline
\end{tabular}


Absolute energies (E), enthalpies (H), RRHO free energies (G), and quasi-RRHO free energies (Grimme_G), in hartrees, along with the corresponding relative values in $\mathrm{kcal} / \mathrm{mol}$ and predicted ee's, for catalyst $3 e$

\begin{tabular}{|c|c|c|c|c|}
\hline Geometry & $\mathrm{E}$ & $\mathrm{H}$ & G & G Grimme \\
\hline $\mathrm{R}$ & $97.1 \%$ & $95.5 \%$ & $80.9 \%$ & $\overline{9} 1.5 \%$ \\
\hline BP1 & -3305.280681 & -3304.709283 & -3304.768833 & -3304.765344 \\
\hline BP2 & -3305.285139 & -3304.712819 & -3304.770448 & -3304.767803 \\
\hline BP 3 & -3305.282508 & -3304.710407 & -3304.768551 & -3304.765586 \\
\hline BP 4 & -3305.272763 & -3304.700762 & -3304.759162 & -3304.756231 \\
\hline BP 5 & -3305.273411 & -3304.701648 & -3304.760519 & -3304.757294 \\
\hline S & & & & \\
\hline BP1 & -3305.280682 & -3304.709282 & -3304.768820 & -3304.765338 \\
\hline BP2 & -3305.282508 & -3304.710408 & -3304.768556 & -3304.765589 \\
\hline BP3 & -3305.279374 & -3304.706851 & -3304.764695 & -3304.761767 \\
\hline BP 4 & -3305.273412 & -3304.701648 & -3304.760521 & -3304.757296 \\
\hline BP 5 & -3305.272763 & -3304.700763 & -3304.759164 & -3304.756233 \\
\hline Relative & Energies & & & \\
\hline Geometry & $\mathrm{E}$ & $\mathrm{H}(r e l)$ & $\mathrm{G}(\mathrm{rel})$ & G_Grimme (rel) \\
\hline $\mathrm{R}$ & $97.1 \%$ & $95.5 \%$ & $80.9 \%$ & $91.5 \%$ \\
\hline BP1 & 2.8 & 2.2 & 1.0 & 1.5 \\
\hline BP2 & 0.0 & 0.0 & 0.0 & 0.0 \\
\hline BP3 & 1.7 & 1.5 & 1.2 & 1.4 \\
\hline BP 4 & 7.8 & 7.6 & 7.1 & 7.3 \\
\hline BP 5 & 7.4 & 7.0 & 6.2 & 6.6 \\
\hline S & & & & \\
\hline BP1 & 2.8 & 2.2 & 1.0 & 1.5 \\
\hline BP2 & 1.7 & 1.5 & 1.2 & 1.4 \\
\hline BP 3 & 3.6 & 3.7 & 3.6 & 3.8 \\
\hline BP 4 & 7.4 & 7.0 & 6.2 & 6.6 \\
\hline BP 5 & 7.8 & 7.6 & 7.1 & 7.3 \\
\hline
\end{tabular}

Absolute energies (E), enthalpies (H), RRHO free energies (G), and quasi-RRHO free energies (Grimme_G), in hartrees, along with the corresponding relative values in $\mathrm{kcal} / \mathrm{mol}$ and predicted ee's, for catalyst $3 f$

\begin{tabular}{|c|c|c|c|c|}
\hline Geometry & $\mathrm{E}$ & $\mathrm{H}$ & G & G_Grimme \\
\hline $\mathrm{R}$ & $89.7 \%$ & $87.8 \%$ & $82.5 \%$ & $\overline{8} 2.9 \%$ \\
\hline BP1 & -2867.106715 & -2866.375565 & -2866.434860 & -2866.431714 \\
\hline BP2 2 & -2867.108737 & -2866.376974 & -2866.434532 & -2866.432061 \\
\hline BP3 & -2867.095929 & -2866.364737 & -2866.422727 & -2866.420049 \\
\hline BP 4 & -2867.098567 & -2866.366561 & -2866.424297 & -2866.421632 \\
\hline BP 5 & -2867.101008 & -2866.368820 & -2866.425869 & -2866.423477 \\
\hline S & & & & \\
\hline BP1 & -2867.104934 & -2866.373422 & -2866.431917 & -2866.429113 \\
\hline BP2 & -2867.106935 & -2866.375320 & -2866.433662 & -2866.430841 \\
\hline BP3 & -2867.101996 & -2866.370513 & -2866.428040 & -2866.425794 \\
\hline $\mathrm{BP} 4$ & -2867.101463 & -2866.369574 & -2866.427496 & -2866.424784 \\
\hline BP 5 & -2867.088198 & -2866.356381 & -2866.414729 & -2866.411752 \\
\hline Relative & Energies & & & \\
\hline Geometry & $E$ & $\mathrm{H}(r \in l)$ & $G(r e l)$ & G_Grim \\
\hline $\mathrm{R}$ & $89.7 \%$ & $87.8 \%$ & $82.5 \%$ & $82.9 \%$ \\
\hline BP1 & 1.3 & 0.9 & 0.0 & 0.2 \\
\hline
\end{tabular}




$\begin{array}{lrrrr}\text { BP2 } & 0.0 & 0.0 & 0.2 & 0.0 \\ \text { BP3 } & 8.0 & 7.7 & 7.6 & 7.5 \\ \text { BP4 } & 6.4 & 6.5 & 6.6 & 6.5 \\ \text { BP5 } & 4.9 & 5.1 & 5.6 & 5.4 \\ \text { S } & & & & \\ \text { BP1 } & 2.4 & 2.2 & 1.8 & 1.8 \\ \text { BP2 } & 1.1 & 1.0 & 0.8 & 0.8 \\ \text { BP3 } & 4.2 & 4.1 & 4.3 & 3.9 \\ \text { BP4 } & 4.6 & 4.6 & 4.6 & 4.6 \\ \text { BP5 } & 12.9 & 12.9 & 12.6 & 12.7\end{array}$

Absolute energies (E), enthalpies (H), RRHO free energies (G), and quasi-RRHO free energies (Grimme_G), in hartrees, along with the corresponding relative values in $\mathrm{kcal} / \mathrm{mol}$ and predicted ee's, for catalyst $3 \mathrm{~h}(\mathrm{R}=\mathrm{Me})$

$\begin{array}{lcccc}\text { Geometry } & \mathrm{E} & \mathrm{H} & \mathrm{G} & \text { G Grimme } \\ \text { R } & 97.5 \% & 96.2 \% & 93.9 \% & \overline{9} \mathbf{9} \\ \text { BP1 } & -2862.147280 & -2861.512491 & -2861.571283 & -2861.568242 \\ \text { BP2 } & -2862.151467 & -2861.516290 & -2861.574034 & -2861.571445 \\ \text { BP3 } & -2862.139255 & -2861.504513 & -2861.562798 & -2861.560104 \\ \text { BP4 } & -2862.141215 & -2861.506209 & -2861.564612 & -2861.561752 \\ \text { BP5 } & -2862.143939 & -2861.508795 & -2861.566325 & -2861.563676 \\ \text { S } & & & & \\ \text { BP1 } & -2862.147457 & -2861.512582 & -2861.570823 & -2861.567984 \\ \text { BP2 } & -2862.148646 & -2861.513753 & -2861.571786 & -2861.569095 \\ \text { BP3 } & -2862.146850 & -2861.511517 & -2861.568710 & -2861.566319 \\ \text { BP4 } & -2862.139529 & -2861.504427 & -2861.562392 & -2861.559711 \\ \text { BP5 } & -2862.139255 & -2861.504513 & -2861.562798 & -2861.560104\end{array}$

Relative Energies

$\begin{array}{lcccc}\text { Geometry } & \text { E } & \text { H }(r e l) & \text { G(rel) } & \text { G_Grimme }(r e l) \\ \text { R } & 97.5 \% & 96.2 \% & 93.9 \% & 94.9 \% \\ \text { BP1 } & 2.6 & 2.4 & 1.7 & 2.0 \\ \text { BP2 } & 0.0 & 0.0 & 0.0 & 0.0 \\ \text { BP3 } & 7.7 & 7.4 & 7.1 & 7.1 \\ \text { BP4 } & 6.4 & 6.3 & 5.9 & 6.1 \\ \text { BP5 } & 4.7 & 4.7 & 4.8 & 4.9 \\ \text { S } & & & & \\ \text { BP1 } & 2.5 & 2.3 & 2.0 & 2.2 \\ \text { BP2 } & 1.8 & 1.6 & 1.4 & 1.5 \\ \text { BP3 } & 2.9 & 3.0 & 3.3 & 7.2 \\ \text { BP4 } & 7.5 & 7.4 & 7.3 & 7.1 \\ \text { BP5 } & 7.7 & 7.4 & 7.1 & \end{array}$

Absolute energies (E), enthalpies (H), RRHO free energies (G), and quasi-RRHO free energies (Grimme_G), in hartrees, along with the corresponding relative values in $\mathrm{kcal} / \mathrm{mol}$ and predicted ee's, for catalyst $3 \mathrm{~h}$

$\begin{array}{lcccc}\text { Geometry } & \text { E } & \text { H } & \text { G } & \text { G Grimme } \\ \text { R } & 96.8 \% & 96.9 \% & 90.1 \% & 94.3 \% \\ \text { BP1 } & -2783.535528 & -2782.956119 & -2783.011578 & -2783.008728 \\ \text { BP2 } & -2783.539351 & -2782.959897 & -2783.014063 & -2783.011812 \\ \text { BP3 } & -2783.527123 & -2782.947824 & -2783.003421 & -2783.000660 \\ \text { BP4 } & -2783.529636 & -2782.950210 & -2783.005637 & -2783.002861 \\ \text { BP5 } & -2783.532117 & -2782.952419 & -2783.006515 & -2783.004224 \\ \text { S } & & & & \\ \text { BP1 } & -2783.535596 & -2782.956337 & -2783.011826 & -2783.008948\end{array}$




\begin{tabular}{lcccc} 
BP2 & -2783.536681 & -2782.957183 & -2783.011809 & -2783.009386 \\
BP3 & -2783.534725 & -2782.955124 & -2783.009186 & -2783.007036 \\
BP4 & -2783.528129 & -2782.948711 & -2783.004196 & -2783.001340 \\
BP5 & -2783.525109 & -2782.945515 & -2783.000760 & -2782.998058 \\
\multicolumn{1}{c}{ Relative Energies } & & & \\
Geometry & E & H (rel) & G(rel) & G_Grimme (rel) \\
R & $96.8 \%$ & $96.9 \%$ & $90.1 \%$ & $94.3 \%$ \\
BP1 & 2.4 & 2.4 & 1.6 & 1.9 \\
BP2 & 0.0 & 0.0 & 0.0 & 0 \\
BP3 & 7.7 & 7.6 & 6.7 & 5.0 \\
BP4 & 6.1 & 6.1 & 5.3 & 4.8 \\
BP5 & 4.5 & 4.7 & 4.7 & 1.8 \\
S & 2.4 & 2.2 & 1.4 & 1.5 \\
BP1 & 1.7 & 1.7 & 1.4 & 3.0 \\
BP2 & 2.9 & 3.0 & 3.1 & 6.6 \\
BP3 & 7.0 & 7.0 & 6.2 & 8.6 \\
BP4 & 8.9 & 9.0 & 8.3 &
\end{tabular}

Absolute energies (E), enthalpies (H), RRHO free energies (G), and quasi-RRHO free energies (Grimme_G), in hartrees, along with the corresponding relative values in $\mathrm{kcal} / \mathrm{mol}$ and predicted ee's, for catalyst $3 i$ ( $\mathrm{R}=\mathrm{Me}$ )

\begin{tabular}{|c|c|c|c|c|}
\hline Geometry & $\mathrm{E}$ & $\mathrm{H}$ & G & G_Grimme \\
\hline $\mathrm{R}$ & $98.9 \%$ & $98.7 \%$ & $99.2 \%$ & $\overline{9} 9.1 \%$ \\
\hline BP1 & -2894.322985 & -2893.708343 & -2893.766388 & -2893.763432 \\
\hline BP2 & -2894.327426 & -2893.712770 & -2893.770303 & -2893.767718 \\
\hline $\mathrm{BP} 3$ & -2894.313843 & -2893.699352 & -2893.757668 & -2893.754687 \\
\hline BP 4 & -2894.314315 & -2893.699910 & -2893.757998 & -2893.755168 \\
\hline BP 5 & -2894.319288 & -2893.704711 & -2893.762073 & -2893.759447 \\
\hline S & & & & \\
\hline $\mathrm{BP} 1$ & -2894.323337 & -2893.708670 & -2893.766094 & -2893.763407 \\
\hline $\mathrm{BP} 2$ & -2894.324056 & -2893.709520 & -2893.766700 & -2893.764270 \\
\hline $\mathrm{BP} 3$ & -2894.322086 & -2893.707207 & -2893.764186 & -2893.761735 \\
\hline BP 4 & -2894.314584 & -2893.699659 & -2893.757895 & -2893.754914 \\
\hline BP 5 & -2894.314165 & -2893.699861 & -2893.758967 & -2893.755713 \\
\hline Relative & Energies & & & \\
\hline Geometry & $\mathrm{E}$ & $\mathrm{H}(r e l)$ & $G(r e l)$ & G_Grimme (rel) \\
\hline $\mathrm{R}$ & $98.9 \%$ & $98.7 \%$ & $99.2 \%$ & $99.1 \%$ \\
\hline BP1 & 2.8 & 2.8 & 2.5 & 2.7 \\
\hline BP2 & 0.0 & 0.0 & 0.0 & 0.0 \\
\hline BP3 & 8.5 & 8.4 & 7.9 & 8.2 \\
\hline BP 4 & 8.2 & 8.1 & 7.7 & 7.9 \\
\hline BP 5 & 5.1 & 5.1 & 5.2 & 5.2 \\
\hline S & & & & \\
\hline BP 1 & 2.6 & 2.6 & 2.6 & 2.7 \\
\hline BP 2 & 2.1 & 2.0 & 2.3 & 2.2 \\
\hline BP3 & 3.4 & 3.5 & 3.8 & 3.8 \\
\hline BP 4 & 8.1 & 8.2 & 7.8 & 8.0 \\
\hline BP 5 & 8.3 & 8.1 & 7.1 & 7.5 \\
\hline
\end{tabular}

Absolute energies (E), enthalpies (H), RRHO free energies (G), and quasi-RRHO free energies (Grimme_G), in hartrees, along with the corresponding relative values in $\mathrm{kcal} / \mathrm{mol}$ and predicted ee's, for catalyst $3 i$ 


\begin{tabular}{|c|c|c|c|c|}
\hline Geometry & $\mathrm{E}$ & $\mathrm{H}$ & G & G_Grimme \\
\hline $\mathrm{R}$ & $98.7 \%$ & $98.3 \%$ & $84.1 \%$ & $\overline{9} 4.4 \%$ \\
\hline BP1 & -2815.709483 & -2815.150650 & -2815.205730 & -2815.202979 \\
\hline BP2 & -2815.713647 & -2815.154529 & -2815.208025 & -2815.205969 \\
\hline BP3 & -2815.700011 & -2815.141066 & -2815.195753 & -2815.193314 \\
\hline BP 4 & -2815.700786 & -2815.141995 & -2815.197566 & -2815.194620 \\
\hline BP 5 & -2815.705624 & -2815.146799 & -2815.201760 & -2815.198985 \\
\hline S & & & & \\
\hline $\mathrm{BP} 1$ & -2815.709753 & -2815.150906 & -2815.206317 & -2815.203376 \\
\hline BP2 & -2815.710334 & -2815.151326 & -2815.205752 & -2815.203329 \\
\hline BP3 & -2815.708096 & -2815.149002 & -2815.202982 & -2815.200742 \\
\hline BP 4 & -2815.701039 & -2815.142164 & -2815.197220 & -2815.194542 \\
\hline BP 5 & -2815.700196 & -2815.141288 & -2815.196412 & -2815.193705 \\
\hline Relative & Energies & & & \\
\hline Geometry & $\mathrm{E}$ & $\mathrm{H}(\mathrm{rel})$ & $G(r e l)$ & G_Grimme(rel) \\
\hline $\mathrm{R}$ & $98.7 \%$ & $98.3 \%$ & $84.1 \%$ & $94.4 \%$ \\
\hline $\mathrm{BP} 1$ & 2.6 & 2.4 & 1.4 & 1.9 \\
\hline BP2 & 0.0 & 0.0 & 0.0 & 0.0 \\
\hline BP3 & 8.6 & 8.4 & 7.7 & 7.9 \\
\hline BP 4 & 8.1 & 7.9 & 6.6 & 7.1 \\
\hline BP 5 & 5.0 & 4.9 & 3.9 & 4.4 \\
\hline S & & & & \\
\hline BP1 & 2.4 & 2.3 & 1.1 & 1.6 \\
\hline BP2 & 2.1 & 2.0 & 1.4 & 1.7 \\
\hline BP3 & 3.5 & 3.5 & 3.2 & 3.3 \\
\hline BP 4 & 7.9 & 7.8 & 6.8 & 7.2 \\
\hline BP 5 & 8.4 & 8.3 & 7.3 & 7.7 \\
\hline
\end{tabular}

Absolute energies (E), enthalpies (H), RRHO free energies (G), and quasi-RRHO free energies (Grimme_G), in hartrees, along with the corresponding relative values in kcal/mol and predicted ee's, for catalyst $3 j$

$\begin{array}{lcccc}\text { Geometry } & \text { E } & \text { H } & \text { G } & \text { G_Grimme } \\ \text { R } & 30.4 \% & -3.3 \% & 61.5 \% & 27.0 \% \\ \text { BP1 } & -3093.218380 & -3092.495253 & -3092.556389 & -3092.552579 \\ \text { BP2 } & -3093.221439 & -3092.497912 & -3092.559227 & -3092.555211 \\ \text { BP3 } & -3093.209066 & -3092.485676 & -3092.545977 & -3092.542439 \\ \text { BP4 } & -3093.211806 & -3092.488836 & -3092.550067 & -3092.546196 \\ \text { BP5 } & -3093.208200 & -3092.485439 & -3092.546583 & -3092.542618 \\ \text { S } & & & & \\ \text { BP1 } & -3093.221048 & -3092.497957 & -3092.558343 & -3092.554874 \\ \text { BP2 } & -3093.218346 & -3092.494926 & -3092.555235 & -3092.551755 \\ \text { BP3 } & -3093.215730 & -3092.492566 & -3092.552669 & -3092.549309 \\ \text { BP4 } & -3093.209605 & -3092.486220 & -3092.546589 & -3092.543060 \\ \text { BP5 } & -3093.208899 & -3092.485859 & -3092.546219 & -3092.542781\end{array}$

Relative Energies

$\begin{array}{lcc}\text { Geometry } & E & \text { H (rel) } \\ \text { R } & 30.4 \% & -3.3 \% \\ \text { BP1 } & 1.9 & 1.7 \\ \text { BP2 } & 0.0 & 0.0 \\ \text { BP3 } & 7.8 & 7.7 \\ \text { BP 4 } & 6.0 & 5.7 \\ \text { BP5 } & 8.3 & 7.9 \\ \text { S } & & \\ \text { BP1 } & 0.2 & 0.0\end{array}$

$\begin{array}{lc}\text { G(rel) } & \text { G_Grimme }(r e l) \\ 61.5 \% & 27.0 \% \\ 1.8 & 1.7 \\ 0.0 & 0.0 \\ 8.3 & 8.0 \\ 5.7 & 5.7 \\ 7.9 & 7.9 \\ 0.6 & 0.2\end{array}$




$\begin{array}{lllll}\text { BP2 } & 1.9 & 1.9 & 2.5 & 2.2 \\ \text { BP3 } & 3.6 & 3.4 & 4.1 & 3.7 \\ \text { BP4 } & 7.4 & 7.4 & 7.9 & 7.6 \\ \text { BP5 } & 7.9 & 7.6 & 8.2 & 7.8\end{array}$

Absolute energies (E), enthalpies (H), RRHO free energies (G), and quasi-RRHO free energies (Grimme G), in hartrees, along with the corresponding relative values in $\mathrm{kcal} / \mathrm{mol}$ and predicted ee's, for catalyst $4 \mathrm{a}(\mathrm{R}=\mathrm{Me})$

$\begin{array}{lcccc}\text { Geometry } & \text { E } & \text { H } & \text { G } & \text { G Grimme } \\ \text { R } & 54.3 \% & 57.8 \% & 88.9 \% & 79.0 \% \\ \text { BP1 } & -2702.673193 & -2702.187589 & -2702.236947 & -2702.234735 \\ \text { BP2 } & -2702.678307 & -2702.192675 & -2702.242895 & -2702.240269 \\ \text { BP3 } & -2702.668447 & -2702.183045 & -2702.233214 & -2702.230695 \\ \text { BP4 } & -2702.668677 & -2702.182903 & -2702.232111 & -2702.229989 \\ \text { BP5 } & -2702.673000 & -2702.187265 & -2702.236365 & -2702.234319 \\ \text { S } & & & & \\ \text { BP1 } & -2702.672067 & -2702.186604 & -2702.236997 & -2702.234343 \\ \text { BP2 } & -2702.677124 & -2702.191580 & -2702.240723 & -2702.238596 \\ \text { BP3 } & -2702.677130 & -2702.191239 & -2702.240709 & -2702.238425 \\ \text { BP4 } & -2702.670038 & -2702.184338 & -2702.233698 & -2702.231511 \\ \text { BP5 } & -2702.665442 & -2702.180200 & -2702.230437 & -2702.228015\end{array}$

Relative Energies

\begin{tabular}{|c|c|c|c|c|}
\hline Geometry & $\mathrm{E}$ & $\mathrm{H}(r \in l)$ & $G(r e l)$ & G_Grimme (rel) \\
\hline $\mathrm{R}$ & $54.3 \%$ & $57.8 \%$ & $88.9 \%$ & $79.0 \%$ \\
\hline BP1 & 3.2 & 3.2 & 3.7 & 3.5 \\
\hline BP2 & 0.0 & 0.0 & 0.0 & 0.0 \\
\hline BP3 & 6.2 & 6.0 & 6.1 & 6.0 \\
\hline BP 4 & 6.0 & 6.1 & 6.8 & 6.5 \\
\hline BP5 & 3.3 & 3.4 & 4.1 & 3.7 \\
\hline \multicolumn{5}{|l|}{$\mathrm{S}$} \\
\hline BP1 & 3.9 & 3.8 & 3.7 & 3.7 \\
\hline BP2 & 0.7 & 0.7 & 1.4 & 1.0 \\
\hline BP3 & 0.7 & 0.9 & 1.4 & 1.2 \\
\hline BP 4 & 5.2 & 5.2 & 5.8 & 5.5 \\
\hline BP5 & 8.1 & 7.8 & 7.8 & 7.7 \\
\hline
\end{tabular}

Absolute energies (E), enthalpies (H), RRHO free energies (G), and quasi-RRHO free energies (Grimme_G), in hartrees, along with the corresponding relative values in $\mathrm{kcal} / \mathrm{mol}$ and predicted ee's, for catalyst $4 \mathrm{a}$

$\begin{array}{lcccc}\text { Geometry } & \text { E } & \text { H } & \text { G } & \text { G Grimme } \\ \text { R } & 24.1 \% & 34.2 \% & 30.5 \% & \overline{3} 5.8 \% \\ \text { BP1 } & -2624.065510 & -2623.635532 & -2623.682457 & -2623.680121 \\ \text { BP2 } & -2624.069578 & -2623.639318 & -2623.685353 & -2623.683379 \\ \text { BP3 } & -2624.059801 & -2623.629712 & -2623.676052 & -2623.674038 \\ \text { BP4 } & -2624.060760 & -2623.630663 & -2623.677004 & -2623.674986 \\ \text { BP5 } & -2624.064900 & -2623.634900 & -2623.680946 & -2623.679026 \\ \text { S } & & & & \\ \text { BP1 } & -2624.064241 & -2623.634066 & -2623.680351 & -2623.678332 \\ \text { BP2 } & -2624.068271 & -2623.638209 & -2623.684821 & -2623.682600 \\ \text { BP3 } & -2624.069140 & -2623.638625 & -2623.684017 & -2623.682359 \\ \text { BP4 } & -2624.062373 & -2623.632529 & -2623.679468 & -2623.677174 \\ \text { BP5 } & -2624.056282 & -2623.626591 & -2623.674606 & -2623.671853\end{array}$

Relative Energies 


$\begin{array}{lcccc}\text { Geometry } & E & H(r e l) & G(r e l) & \text { G_Grimme }(r e l) \\ \text { R } & 24.1 \% & 34.2 \% & 30.5 \% & 35.8 \% \\ \text { BP1 } & 2.6 & 2.4 & 1.8 & 2.0 \\ \text { BP2 } & 0.0 & 0.0 & 0.0 & 0.0 \\ \text { BP3 } & 6.1 & 6.0 & 5.8 & 5.9 \\ \text { BP4 } & 5.5 & 2.4 & 5.2 & 5.3 \\ \text { BP5 } & 2.9 & 3.8 & 2.8 & 2.7 \\ \text { S } & & & 3.1 & 3.2 \\ \text { BP1 } & 3.3 & 0.7 & 0.3 & 0.5 \\ \text { BP2 } & 0.8 & 0.4 & 0.8 & 0.6 \\ \text { BP3 } & 0.3 & 4.3 & 3.7 & 3.9 \\ \text { BP4 } & 4.5 & 8.0 & 6.7 & 7.2 \\ \text { BP5 } & 8.3 & & & \end{array}$

Absolute energies (E), enthalpies (H), RRHO free energies (G), and quasi-RRHO free energies (Grimme_G), in hartrees, along with the corresponding relative values in $\mathrm{kcal} / \mathrm{mol}$ and predicted ee's, for catalyst $4 \mathrm{~b}$

\begin{tabular}{|c|c|c|c|c|}
\hline Geometry & $\mathrm{E}$ & $\mathrm{H}$ & G & G Grimme \\
\hline $\mathrm{R}$ & $71.0 \%$ & $74.8 \%$ & $78.8 \%$ & $\overline{7} 7.7 \%$ \\
\hline BP1 & -2822.501776 & -2822.087739 & -2822.136311 & -2822.133902 \\
\hline BP2 & -2822.503603 & -2822.089705 & -2822.138213 & -2822.135793 \\
\hline BP3 & -2822.491934 & -2822.078085 & -2822.127160 & -2822.124528 \\
\hline BP 4 & -2822.492443 & -2822.078340 & -2822.126880 & -2822.124528 \\
\hline BP5 & -2822.497252 & -2822.083296 & -2822.131810 & -2822.129446 \\
\hline $\mathrm{S}$ & & & & \\
\hline BP1 & -2822.500989 & -2822.087208 & -2822.136269 & -2822.133656 \\
\hline BP2 & -2822.502470 & -2822.088443 & -2822.136648 & -2822.134355 \\
\hline BP3 & -2822.500231 & -2822.086187 & -2822.134309 & -2822.132104 \\
\hline BP 4 & -2822.492926 & -2822.078950 & -2822.128036 & -2822.125459 \\
\hline BP5 & -2822.489238 & -2822.075394 & -2822.124312 & -2822.121839 \\
\hline Relative & Energies & & & \\
\hline Geometry & E & $\mathrm{H}(\mathrm{rel})$ & $G(r e l)$ & G_Grimme (rel) \\
\hline $\mathrm{R}$ & $71.0 \%$ & $74.8 \%$ & $78.8 \%$ & $77.7 \%$ \\
\hline BP1 & 1.1 & 1.2 & 1.2 & 1.2 \\
\hline BP2 & 0.0 & 0.0 & 0.0 & 0.0 \\
\hline BP3 & 7.3 & 7.3 & 6.9 & 7.1 \\
\hline BP 4 & 7.0 & 7.1 & 7.1 & 7.1 \\
\hline BP5 & 4.0 & 4.0 & 4.0 & 4.0 \\
\hline S & & & & \\
\hline BP1 & 1.6 & 1.6 & 1.2 & 1.3 \\
\hline BP2 & 0.7 & 0.8 & 1.0 & 0.9 \\
\hline BP3 & 2.1 & 2.2 & 2.4 & 2.3 \\
\hline BP 4 & 6.7 & 6.7 & 6.4 & 6.5 \\
\hline BP 5 & 9.0 & 9.0 & 8.7 & 8 \\
\hline
\end{tabular}

Absolute energies (E), enthalpies (H), RRHO free energies (G), and quasi-RRHO free energies (Grimme_G), in hartrees, along with the corresponding relative values in $\mathrm{kcal} / \mathrm{mol}$ and predicted ee's, for catalyst $4 \mathrm{c}$

$\begin{array}{lcccc}\text { Geometry } & \mathrm{E} & \mathrm{H} & \mathrm{G} & \mathrm{G} \text { Grimme } \\ \text { R } & 75.4 \% & 78.0 \% & 70.7 \% & \overline{7} .9 \% \\ \text { BP1 } & -3543.292425 & -3542.881129 & -3542.931294 & -3542.928605 \\ \text { BP2 } & -3543.295078 & -3542.883684 & -3542.933212 & -3542.930755 \\ \text { BP3 } & -3543.283998 & -3542.872515 & -3542.922338 & -3542.919779 \\ \text { BP4 } & -3543.280773 & -3542.869018 & -3542.918902 & -3542.916327\end{array}$




$\begin{array}{lcccc}\text { BP5 } & -3543.287559 & -3542.876221 & -3542.925510 & -3542.923203 \\ \text { S } & & & & \\ \text { BP1 } & -3543.292334 & -3542.881092 & -3542.931243 & -3542.928541 \\ \text { BP2 } & -3543.293812 & -3542.882319 & -3542.931985 & -3542.929476 \\ \text { BP3 } & -3543.291199 & -3542.879516 & -3542.928684 & -3542.926433 \\ \text { BP4 } & -3543.281828 & -3542.870261 & -3542.919747 & -3542.917385 \\ \text { BP5 } & -3543.281404 & -3542.870169 & -3542.920314 & -3542.917667 \\ \text { Relative Energies } & & & \\ \text { Geometry } & \text { E } & \text { H rel) } & \text { G(rel) } & \text { G_Grimme (rel) } \\ \text { R } & 75.4 \% & 78.0 \% & 70.7 \% & 73.9 \% \\ \text { BP1 } & 1.7 & 1.6 & 1.2 & 0.3 \\ \text { BP2 } & 0.0 & 0.0 & 0.0 & 6.9 \\ \text { BP3 } & 7.0 & 7.0 & 6.8 & 9.1 \\ \text { BP4 } & 9.0 & 9.2 & 9.0 & 4.7 \\ \text { BP5 } & 4.7 & 4.7 & 4.8 & 1.4 \\ \text { S } & 1.7 & 1.6 & 1.2 & 0.8 \\ \text { BP1 } & 0.8 & 0.9 & 0.8 & 2.7 \\ \text { BP2 } & 2.4 & 2.6 & 2.8 & 8.4 \\ \text { BP3 } & 8.3 & 8.4 & 8.4 & 8.2 \\ \text { BP4 } & 8.6 & 8.5 & 8.1 & \\ \text { BP5 } & & & & 0\end{array}$

Absolute energies (E), enthalpies (H), RRHO free energies (G), and quasi-RRHO free energies (Grimme_G), in hartrees, along with the corresponding relative values in $\mathrm{kcal} / \mathrm{mol}$ and predicted ee's, for catalyst $4 \mathrm{~d}$

$\begin{array}{lcccc}\text { Geometry } & \text { E } & \text { H } & \text { G } & \text { G_Grimme } \\ \text { R } & -45.2 \% & -28.6 \% & 8.3 \% & -8.0 \% \\ \text { BP1 } & -2702.655607 & -2702.169928 & -2702.219161 & -2702.216789 \\ \text { BP2 } & -2702.656528 & -2702.171235 & -2702.220237 & -2702.217991 \\ \text { BP3 } & -2702.647788 & -2702.162422 & -2702.211570 & -2702.209326 \\ \text { BP4 } & -2702.646561 & -2702.160401 & -2702.209531 & -2702.207111 \\ \text { BP5 } & -2702.651416 & -2702.165834 & -2702.214856 & -2702.212629 \\ \text { S } & & & & \\ \text { BP1 } & -2702.652066 & -2702.166785 & -2702.216451 & -2702.214001 \\ \text { BP2 } & -2702.657252 & -2702.171665 & -2702.220226 & -2702.218166 \\ \text { BP3 } & -2702.654043 & -2702.168533 & -2702.217387 & -2702.215309 \\ \text { BP4 } & -2702.648086 & -2702.162571 & -2702.211571 & -2702.209322 \\ \text { BP5 } & -2702.644902 & -2702.159143 & -2702.208453 & -2702.206023\end{array}$

Relative Energies

$\begin{array}{lcccc}\text { Geometry } & \text { E } & \text { H (rel) } & \text { G(rel) } & \text { G_Grimme (rel) } \\ \text { R } & -45.2 \% & -28.6 \% & 8.3 \% & -8.0 \% \\ \text { BP1 } & 1.0 & 1.1 & 0.7 & 0.9 \\ \text { BP2 } & 0.5 & 0.3 & 0.0 & 0.1 \\ \text { BP3 } & 5.9 & 5.8 & 5.4 & 5.5 \\ \text { BP4 } & 6.7 & 7.1 & 6.7 & 6.9 \\ \text { BP5 } & 3.7 & 3.7 & 3.4 & 3.5 \\ \text { S } & & & & 2.6 \\ \text { BP1 } & 3.3 & 3.1 & 2.4 & 0.0 \\ \text { BP2 } & 0.0 & 0.0 & 0.0 & 1.8 \\ \text { BP3 } & 2.0 & 2.0 & 1.8 & 5.5 \\ \text { BP4 } & 5.8 & 5.7 & 5.4 & 7.6 \\ \text { BP5 } & 7.7 & 7.9 & 7.4 & \end{array}$




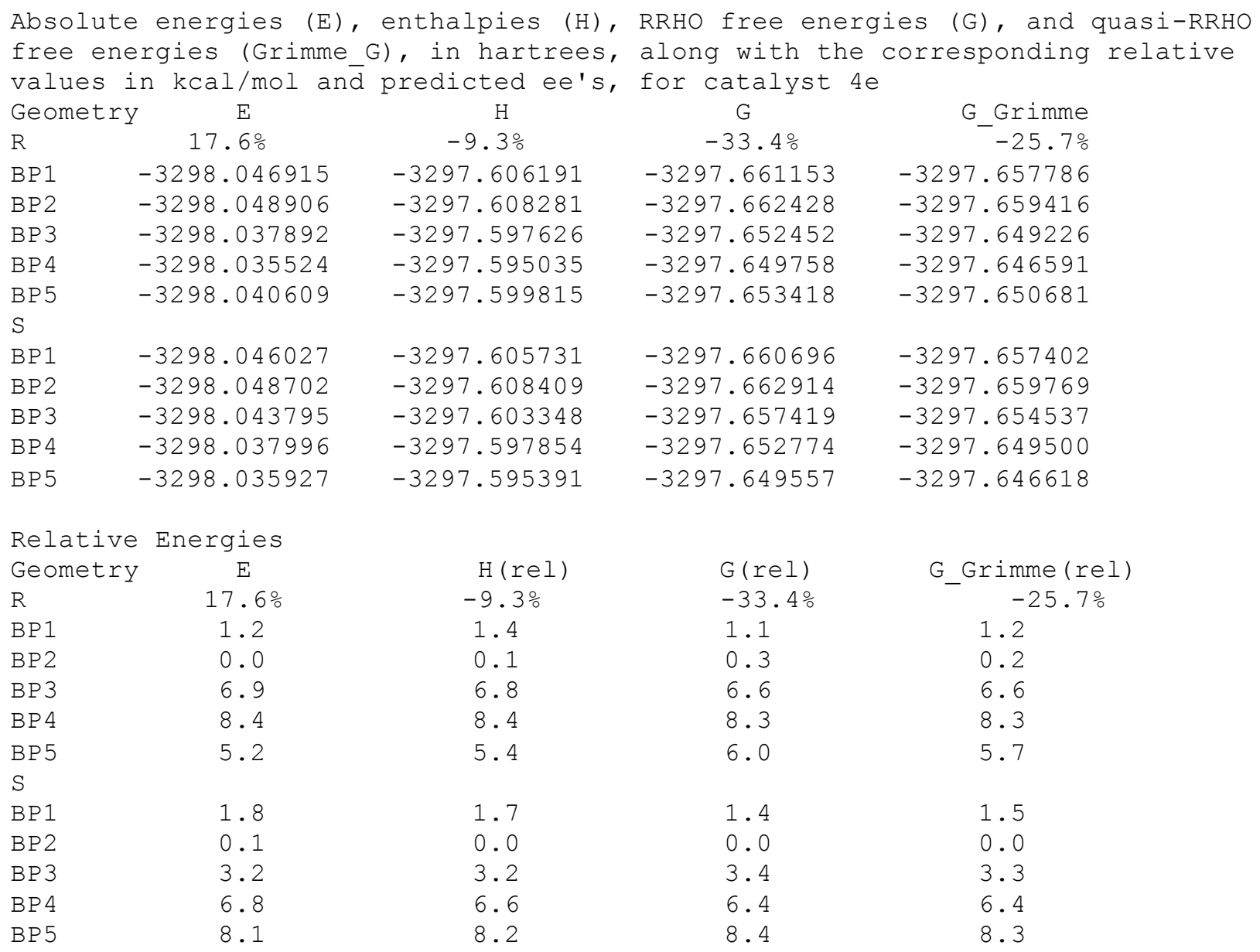

Absolute energies (E), enthalpies (H), RRHO free energies (G), and quasi-RRHO free energies (Grimme G), in hartrees, along with the corresponding relative values in $\mathrm{kcal} / \mathrm{mol}$ and predicted ee's, for catalyst $4 \mathrm{f}$

$\begin{array}{lcccc}\text { Geometry } & \text { E } & \text { H } & \text { G } & \text { G_Grimme } \\ \text { R } & -27.6 \% & -6.3 \% & 24.3 \% & 9.8 \% \\ \text { BP1 } & -2859.851451 & -2859.252558 & -2859.306723 & -2859.303976 \\ \text { BP2 } & -2859.854616 & -2859.256207 & -2859.310600 & -2859.307690 \\ \text { BP3 } & -2859.845619 & -2859.247289 & -2859.301559 & -2859.298817 \\ \text { BP4 } & -2859.843711 & -2859.244498 & -2859.298219 & -2859.295593 \\ \text { BP5 } & -2859.849309 & -2859.250437 & -2859.304299 & -2859.301582 \\ \text { S } & & & & \\ \text { BP1 } & -2859.851380 & -2859.252867 & -2859.308037 & -2859.304856 \\ \text { BP2 } & -2859.854962 & -2859.256280 & -2859.310276 & -2859.307559 \\ \text { BP3 } & -2859.852018 & -2859.253202 & -2859.306847 & -2859.304359 \\ \text { BP4 } & -2859.845130 & -2859.246080 & -2859.300146 & -2859.297335 \\ \text { BP5 } & -2859.828724 & -2859.229739 & -2859.283133 & -2859.280691\end{array}$

Relative Energies

$\begin{array}{lcccc}\text { Geometry } & E & \text { H }(r e l) & \text { G(rel) } & \text { G_Grimme (rel) } \\ \text { R } & -27.6 \% & -6.3 \% & 24.3 \% & 9.8 \% \\ \text { BP1 } & 2.2 & 2.3 & 2.4 & 2.3 \\ \text { BP2 } & 0.2 & 0.0 & 0.0 & 0.0 \\ \text { BP3 } & 5.9 & 5.6 & 5.7 & 5.6 \\ \text { BP } & 7.1 & 7.4 & 7.8 & 7.6\end{array}$




$\begin{array}{lrrrr}\text { BP5 } & 3.5 & 3.7 & 4.0 & 3.8 \\ \text { S } & & & & \\ \text { BP1 } & 2.2 & 2.1 & 1.6 & 1.8 \\ \text { BP2 } & 0.0 & 0.0 & 0.2 & 0.1 \\ \text { BP3 } & 1.8 & 1.9 & 2.4 & 2.1 \\ \text { BP4 } & 6.2 & 6.4 & 6.6 & 6.5 \\ \text { BP5 } & 16.5 & 16.7 & 17.2 & 16.9\end{array}$

Absolute energies (E), enthalpies (H), RRHO free energies (G), and quasi-RRHO free energies (Grimme_G), in hartrees, along with the corresponding relative values in $\mathrm{kcal} / \mathrm{mol}$ and predicted ee's, for catalyst $4 \mathrm{~g}$

$\begin{array}{lcccc}\text { Geometry } & \text { E } & \text { H } & \text { G } & \text { G Grimme } \\ \text { R } & 75.4 \% & 69.2 \% & 27.0 \% & 42.8 \% \\ \text { BP1 } & -2938.418429 & -2937.764776 & -2937.821336 & -2937.818167 \\ \text { BP2 } & -2938.420231 & -2937.766645 & -2937.822316 & -2937.819492 \\ \text { BP3 } & -2938.413322 & -2937.759402 & -2937.815489 & -2937.812490 \\ \text { BP4 } & -2938.408219 & -2937.753477 & -2937.808819 & -2937.806065 \\ \text { BP5 } & -2938.414011 & -2937.759967 & -2937.815215 & -2937.812621 \\ \text { S } & & & & \\ \text { BP1 } & -2938.417073 & -2937.763377 & -2937.819705 & -2937.816664 \\ \text { BP2 } & -2938.419022 & -2937.765605 & -2937.822076 & -2937.818980 \\ \text { BP3 } & -2938.415291 & -2937.761417 & -2937.817223 & -2937.814313 \\ \text { BP4 } & -2938.412079 & -2937.758185 & -2937.813907 & -2937.811083 \\ \text { BP5 } & -2938.413859 & -2937.759712 & -2937.815080 & -2937.812372\end{array}$

Relative Energies

$\begin{array}{lcccc}\text { Geometry } & E & \text { H }(r e l) & \text { G }(r e l) & \text { G_Grimme }(r e l) \\ \text { R } & 75.4 \% & 69.2 \% & 27.0 \% & 42.8 \% \\ \text { BP1 } & 1.1 & 1.2 & 0.6 & 0.8 \\ \text { BP2 } & 0.0 & 0.0 & 0.0 & 0.0 \\ \text { BP3 } & 4.3 & 4.5 & 4.3 & 4.4 \\ \text { BP4 } & 7.5 & 4.3 & 8.5 & 8.4 \\ \text { BP5 } & 3.9 & & & 4.3 \\ \text { S } & & 2.1 & 1.6 & 1.8 \\ \text { BP1 } & 2.0 & 0.7 & 0.2 & 0.3 \\ \text { BP2 } & 0.8 & 3.3 & 3.2 & 3.2 \\ \text { BP3 } & 3.1 & 5.3 & 5.3 & 5.3 \\ \text { BP4 } & 5.1 & 4.4 & 4.5 & 4.5 \\ \text { BP5 } & 4.0 & & & \end{array}$

Absolute energies (E), enthalpies (H), RRHO free energies (G), and quasi-RRHO free energies (Grimme_G), in hartrees, along with the corresponding relative values in $\mathrm{kcal} / \mathrm{mol}$ and predicted ee's, for catalyst $4 \mathrm{~h}$

$\begin{array}{lcccc}\text { Geometry } & \mathrm{E} & \mathrm{H} & \mathrm{G} & \text { G Grimme } \\ \text { R } & 51.8 \% & 44.2 \% & 31.5 \% & 35.3 \% \\ \text { BP1 } & -2776.268618 & -2775.820730 & -2775.871702 & -2775.869137 \\ \text { BP2 } & -2776.269333 & -2775.821100 & -2775.871306 & -2775.869045 \\ \text { BP3 } & -2776.258117 & -2775.810112 & -2775.860715 & -2775.858312 \\ \text { BP4 } & -2776.253264 & -2775.805367 & -2775.856551 & -2775.853960 \\ \text { BP5 } & -2776.259596 & -2775.811806 & -2775.863028 & -2775.860348 \\ \text { S } & & & & \\ \text { BP1 } & -2776.268715 & -2775.820712 & -2775.871471 & -2775.868983 \\ \text { BP2 } & -2776.267455 & -2775.819400 & -2775.870317 & -2775.867760 \\ \text { BP3 } & -2776.265619 & -2775.817267 & -2775.867625 & -2775.865340 \\ \text { BP4 } & -2776.258367 & -2775.810220 & -2775.860416 & -2775.858187\end{array}$




\begin{tabular}{|c|c|c|c|c|}
\hline BP 5 & -2776.256105 & -2775.808239 & -2775.859462 & -2775.856890 \\
\hline \multicolumn{5}{|c|}{ Relative Energies } \\
\hline Geometry & $E$ & $\mathrm{H}(\mathrm{rel})$ & $G(r e l)$ & G_Grimme (rel) \\
\hline $\mathrm{R}$ & $51.8 \%$ & $44.2 \%$ & $31.5 \%$ & $-35.3 \%$ \\
\hline BP1 & 0.4 & 0.2 & 0.0 & 0.0 \\
\hline BP2 & 0.0 & 0.0 & 0.2 & 0.1 \\
\hline $\mathrm{BP} 3$ & 7.0 & 6.9 & 6.9 & 6.8 \\
\hline BP 4 & 10.1 & 9.9 & 9.5 & 9.5 \\
\hline BP 5 & 6.1 & 5.8 & 5.4 & 5.5 \\
\hline \multicolumn{5}{|l|}{ S } \\
\hline BP1 & 0.4 & 0.2 & 0.1 & 0.1 \\
\hline BP2 & 1.2 & 1.1 & 0.9 & 0.9 \\
\hline BP3 & 2.3 & 2.4 & 2.6 & 2.4 \\
\hline BP 4 & 6.9 & 6.8 & 7.1 & 6.9 \\
\hline BP 5 & 8.3 & 8.1 & 7.7 & 7.7 \\
\hline
\end{tabular}

Absolute energies (E), enthalpies (H), RRHO free energies (G), and quasi-RRHO free energies (Grimme_G), in hartrees, along with the corresponding relative values in $\mathrm{kcal} / \mathrm{mol}$ and predicted ee's, for catalyst $4 i$

$\begin{array}{lcccc}\text { Geometry } & \text { E } & \text { H } & \text { G } & \text { G Grimme } \\ \text { R } & 88.3 \% & 85.8 \% & 77.6 \% & 79.6 \% \\ \text { BP1 } & -2808.458044 & -2808.030144 & -2808.080734 & -2808.078199 \\ \text { BP2 } & -2808.459009 & -2808.030631 & -2808.080425 & -2808.078162 \\ \text { BP3 } & -2808.447329 & -2808.019209 & -2808.069265 & -2808.066953 \\ \text { BP4 } & -2808.442894 & -2808.015091 & -2808.066748 & -2808.063802 \\ \text { BP5 } & -2808.449584 & -2808.021540 & -2808.071759 & -2808.069396 \\ \text { S } & & & & \\ \text { BP1 } & -2808.456282 & -2808.028272 & -2808.078560 & -2808.076210 \\ \text { BP2 } & -2808.457268 & -2808.029113 & -2808.079645 & -2808.077128 \\ \text { BP3 } & -2808.455538 & -2808.027132 & -2808.076881 & -2808.074725 \\ \text { BP4 } & -2808.448164 & -2808.020288 & -2808.070716 & -2808.068276 \\ \text { BP5 } & -2808.443488 & -2808.015612 & -2808.066643 & -2808.064024\end{array}$

\begin{tabular}{|c|c|c|c|c|}
\hline \multicolumn{5}{|c|}{ Relative Energies } \\
\hline Geometry & $E$ & $\mathrm{H}(\mathrm{rel})$ & $G(r e l)$ & G_Grimme(rel) \\
\hline $\mathrm{R}$ & $88.3 \%$ & $85.8 \%$ & $77.6 \%$ & $79.6 \%$ \\
\hline $\mathrm{BP} 1$ & 0.6 & 0.3 & 0.0 & 0.0 \\
\hline BP2 & 0.0 & 0.0 & 0.2 & 0.0 \\
\hline BP3 & 7.3 & 7.2 & 7.2 & 7.1 \\
\hline BP 4 & 10.1 & 9.8 & 8.8 & 9.0 \\
\hline BP 5 & 5.9 & 5.7 & 5.6 & 5.5 \\
\hline \multicolumn{5}{|l|}{ S } \\
\hline BP 1 & 1.7 & 1.5 & 1.4 & 1.2 \\
\hline BP 2 & 1.1 & 1.0 & 0.7 & 0.7 \\
\hline $\mathrm{BP} 3$ & 2.2 & 2.2 & 2.4 & 2.2 \\
\hline BP 4 & 6.8 & 6.5 & 6.3 & 6.2 \\
\hline BP 5 & 9.7 & 9.4 & 8.8 & 8.9 \\
\hline
\end{tabular}

Absolute energies (E), enthalpies (H), RRHO free energies (G), and quasi-RRHO free energies (Grimme_G), in hartrees, along with the corresponding relative values in kcal/mol and predicted ee's, for catalyst $4 j$

$\begin{array}{lcccc}\text { Geometry } & \mathrm{E} & \mathrm{H} & \mathrm{G} & \mathrm{G} \text { Grimme } \\ \mathrm{R} & 86.6 \% & 73.3 \% & 39.9 \% & 54.4 \% \\ \mathrm{BP} 1 & -3085.959650 & -3085.368076 & -3085.423135 & -3085.420293\end{array}$




$\begin{array}{lcccc}\text { BP2 } & -3085.958210 & -3085.367054 & -3085.422728 & -3085.419582 \\ \text { BP3 } & -3085.947848 & -3085.356744 & -3085.412375 & -3085.409271 \\ \text { BP4 } & -3085.94486 & -3085.353802 & -3085.409717 & -3085.406572 \\ \text { BP5 } & -3085.937650 & -3085.346377 & -3085.402053 & -3085.398897 \\ \text { S } & & & & \\ \text { BP1 } & -3085.957740 & -3085.366687 & -3085.422674 & -3085.419458 \\ \text { BP2 } & -3085.957547 & -3085.366500 & -3085.422068 & -3085.419032 \\ \text { BP3 } & -3085.954488 & -3085.363095 & -3085.418383 & -3085.415450 \\ \text { BP4 } & -3085.945411 & -3085.354257 & -3085.410009 & -3085.406812 \\ \text { BP5 } & -3085.935256 & -3085.343825 & -3085.398689 & -3085.395920 \\ & & & & \\ \text { Relative Energies } & \text { E } & \text { H(rel) } & \text { G(rel) } & \text { G_Grimme (rel) } \\ \text { Geometry } & 86.6 \% & 73.3 \% & 39.9 \% & 54.4 \% \\ \text { R } & 0.0 & 0.0 & 0.0 & 0.0 \\ \text { BP1 } & 0.9 & 0.6 & 0.3 & 6.9 \\ \text { BP2 } & 7.4 & 7.1 & 6.8 & 8.6 \\ \text { BP3 } & 9.3 & 9.0 & 8.4 & 13.4 \\ \text { BP4 } & 13.8 & 13.6 & 13.2 & 0.5 \\ \text { BP5 } & & & & 0.8 \\ \text { S } & 1.2 & 1.0 & 0.9 & 3.0 \\ \text { BP1 } & 1.3 & 3.1 & 8.0 & 8.5 \\ \text { BP2 } & 3.2 & 15.2 & 15.3 & 15.3 \\ \text { BP3 } & 8.9 & & & \end{array}$

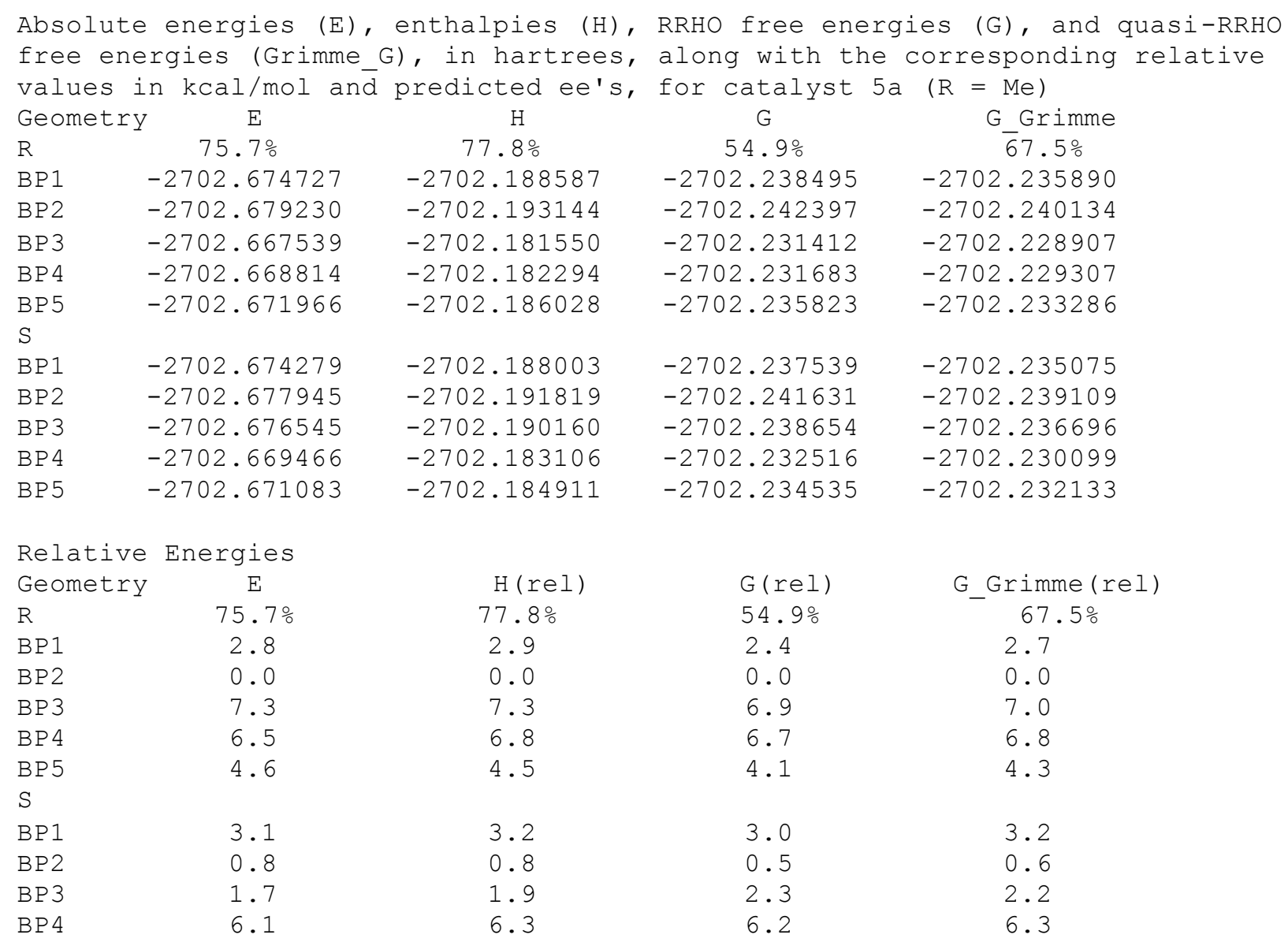


$\begin{array}{lllll}\text { BP5 } & 5.1 & 5.2 & 4.9 & 5.0\end{array}$

Absolute energies (E), enthalpies (H), RRHO free energies (G), and quasi-RRHO free energies (Grimme_G), in hartrees, along with the corresponding relative values in $\mathrm{kcal} / \mathrm{mol}$ and predicted ee's, for catalyst $5 \mathrm{a}$

\begin{tabular}{|c|c|c|c|c|}
\hline Geometry & $\mathrm{E}$ & $\mathrm{H}$ & G & G Grimme \\
\hline $\mathrm{R}$ & $77.5 \%$ & $72.5 \%$ & $45.9 \%$ & $\overline{6} 0.0 \%$ \\
\hline BP1 & -2624.065239 & -2623.635223 & -2623.682329 & -2623.679994 \\
\hline BP2 & -2624.068770 & -2623.638572 & -2623.684925 & -2623.682871 \\
\hline BP 3 & -2624.057419 & -2623.627531 & -2623.675046 & -2623.672537 \\
\hline $\mathrm{BP} 4$ & -2624.059025 & -2623.628962 & -2623.675984 & -2623.673708 \\
\hline BP 5 & -2624.062139 & -2623.632251 & -2623.679549 & -2623.677105 \\
\hline S & & & & \\
\hline BP1 & -2624.064714 & -2623.634655 & -2623.681676 & -2623.679387 \\
\hline BP2 2 & -2624.067371 & -2623.637340 & -2623.684143 & -2623.681880 \\
\hline BP3 & -2624.066415 & -2623.636228 & -2623.683431 & -2623.680996 \\
\hline BP 4 & -2624.059790 & -2623.630147 & -2623.678062 & -2623.675364 \\
\hline BP 5 & -2624.061332 & -2623.631343 & -2623.678198 & -2623.675977 \\
\hline Relative & Energies & & & \\
\hline Geometry & $E$ & $\mathrm{H}(r e l)$ & $\mathrm{G}(\mathrm{rel})$ & G_Grimme (rel) \\
\hline $\mathrm{R}$ & $77.5 \%$ & $72.5 \%$ & $45.9 \%$ & $60.0 \%$ \\
\hline BP1 & 2.2 & 2.1 & 1.6 & 1.8 \\
\hline BP2 & 0.0 & 0.0 & 0.0 & 0.0 \\
\hline BP3 & 7.1 & 6.9 & 6.2 & 6.5 \\
\hline BP 4 & 6.1 & 6.0 & 5.6 & 5.7 \\
\hline BP 5 & 4.2 & 4.0 & 3.4 & 3.6 \\
\hline S & & & & \\
\hline BP1 & 2.5 & 2.5 & 2.0 & 2.2 \\
\hline BP2 & 0.9 & 0.8 & 0.5 & 0.6 \\
\hline BP 3 & 1.5 & 1.5 & 0.9 & 1.2 \\
\hline BP 4 & 5.6 & 5.3 & 4.3 & 4.7 \\
\hline BP5 & 4.7 & 4.5 & 4.2 & 4.3 \\
\hline
\end{tabular}

Absolute energies (E), enthalpies (H), RRHO free energies (G), and quasi-RRHO free energies (Grimme_G), in hartrees, along with the corresponding relative values in $\mathrm{kcal} / \mathrm{mol}$ and predicted ee's, for catalyst 5b

$\begin{array}{lcccc}\text { Geometry } & \text { E } & \text { H } & \text { G } & \text { G_Grimme } \\ \text { R } & 89.9 \% & 88.3 \% & 78.0 \% & 83.6 \% \\ \text { BP1 } & -2822.511542 & -2822.097106 & -2822.146441 & -2822.143786 \\ \text { BP2 } & -2822.515234 & -2822.100439 & -2822.148579 & -2822.146411 \\ \text { BP3 } & -2822.503106 & -2822.088494 & -2822.137375 & -2822.134946 \\ \text { BP4 } & -2822.505088 & -2822.090347 & -2822.139106 & -2822.136716 \\ \text { BP5 } & -2822.508590 & -2822.094058 & -2822.143188 & -2822.140573 \\ \text { S } & & & & \\ \text { BP1 } & -2822.511358 & -2822.096941 & -2822.145923 & -2822.143453 \\ \text { BP2 } & -2822.513006 & -2822.098421 & -2822.147160 & -2822.144726 \\ \text { BP3 } & -2822.512942 & -2822.098042 & -2822.145928 & -2822.143899 \\ \text { BP 4 } & -2822.505060 & -2822.090800 & -2822.140212 & -2822.137554 \\ \text { BP5 } & -2822.506574 & -2822.091747 & -2822.140290 & -2822.138016\end{array}$

Relative Energies

$\begin{array}{lc}\text { Geometry } & E \\ \text { R } & 89.9 \% \\ \text { BP1 } & 2.3\end{array}$

$\begin{array}{clc}\mathrm{H}(\mathrm{rel}) & \mathrm{G}(\mathrm{rel}) & \text { G_Grimme }(r e l) \\ 88.3 \% & 78.0 \% & 83.6 \% \\ 2.1 & 1.3 & 1.6\end{array}$




$\begin{array}{lllll}\text { BP2 } & 0.0 & 0.0 & 0.0 & 0.0 \\ \text { BP3 } & 7.6 & 7.5 & 7.0 & 7.2 \\ \text { BP4 } & 6.4 & 6.3 & 5.9 & 6.1 \\ \text { BP5 } & 4.2 & 4.0 & 3.4 & 3.7 \\ \text { S } & & & & \\ \text { BP1 } & 2.4 & 2.2 & 1.7 & 1.9 \\ \text { BP2 } & 1.4 & 1.3 & 0.9 & 1.1 \\ \text { BP3 } & 1.4 & 1.5 & 1.7 & 5.6 \\ \text { BP4 } & 6.4 & 6.0 & 5.3 & 5.3 \\ \text { BP5 } & 5.4 & 5.5 & 5.2 & \end{array}$

Absolute energies (E), enthalpies (H), RRHO free energies (G), and quasi-RRHO free energies (Grimme_G), in hartrees, along with the corresponding relative values in $\mathrm{kcal} / \mathrm{mol}$ and predicted ee's, for catalyst $5 \mathrm{c}$

$\begin{array}{lcccc}\text { Geometry } & \text { E } & \text { H } & \text { G } & \text { G_Grimme } \\ \text { R } & 91.7 \% & 88.3 \% & 89.4 \% & 88.3 \% \\ \text { BP1 } & -3543.307693 & -3542.895733 & -3542.945455 & -3542.942995 \\ \text { BP2 } & -3543.311013 & -3542.898952 & -3542.948573 & -3542.946090 \\ \text { BP3 } & -3543.298827 & -3542.887097 & -3542.937259 & -3542.934640 \\ \text { BP4 } & -3543.301061 & -3542.889077 & -3542.938843 & -3542.936376 \\ \text { BP5 } & -3543.304469 & -3542.892443 & -3542.941776 & -3542.939468 \\ \text { S } & & & & \\ \text { BP1 } & -3543.307320 & -3542.895379 & -3542.945028 & -3542.942601 \\ \text { BP2 } & -3543.308761 & -3542.897088 & -3542.946686 & -3542.944252 \\ \text { BP3 } & -3543.308425 & -3542.896142 & -3542.945421 & -3542.943111 \\ \text { BP4 } & -3543.301515 & -3542.889662 & -3542.939526 & -3542.937004 \\ \text { BP5 } & -3543.297488 & -3542.885812 & -3542.936378 & -3542.933613\end{array}$

$\begin{array}{lcccc}\text { Relative } & \text { Energies } & & \\ \text { Geometry } & \text { E } & \text { H }(r e l) & G(r e l) & \text { G_Grimme }(r e l) \\ \text { R } & 91.7 \% & 88.3 \% & 89.4 \% & 88.3 \% \\ \text { BP1 } & 2.1 & 2.0 & 2.0 & 1.9 \\ \text { BP2 } & 0.0 & 0.0 & 0.0 & 0.0 \\ \text { BP3 } & 7.6 & 6.4 & 7.1 & 7.2 \\ \text { BP4 } & 6.2 & 4.1 & 6.1 & 6.1 \\ \text { BP5 } & 4.1 & & 4.3 & 4.2 \\ \text { S } & & 2.2 & 2.2 & \\ \text { BP1 } & 2.3 & 1.2 & 1.2 & 1.2 \\ \text { BP2 } & 1.4 & 1.8 & 2.0 & 1.9 \\ \text { BP3 } & 1.6 & 5.8 & 5.7 & 5.7 \\ \text { BP4 } & 6.0 & 8.2 & 7.7 & 7.8 \\ \text { BP5 } & 8.5 & & & \end{array}$

Absolute energies (E), enthalpies (H), RRHO free energies (G), and quasi-RRHO free energies (Grimme_G), in hartrees, along with the corresponding relative values in $\mathrm{kcal} / \mathrm{mol}$ and predicted ee's, for catalyst $5 \mathrm{~d}$

$\begin{array}{lcccc}\text { Geometry } & \text { E } & \text { H } & \text { G } & \text { G_Grimme } \\ \text { R } & 79.1 \% & 62.2 \% & 41.7 \% & 40.8 \% \\ \text { BP1 } & -2702.681931 & -2702.196291 & -2702.246300 & -2702.243893 \\ \text { BP2 } & -2702.685550 & -2702.199297 & -2702.248660 & -2702.246233 \\ \text { BP3 } & -2702.673511 & -2702.187539 & -2702.237613 & -2702.235136 \\ \text { BP4 } & -2702.675281 & -2702.189321 & -2702.239067 & -2702.236684 \\ \text { BP5 } & -2702.679641 & -2702.193468 & -2702.242160 & -2702.240147 \\ \text { S } & & & & \\ \text { BP1 } & -2702.681310 & -2702.195657 & -2702.245324 & -2702.243041\end{array}$




\begin{tabular}{|c|c|c|c|c|}
\hline BP2 & -2702.684049 & -2702.198304 & -2702.248086 & -2702.245657 \\
\hline BP3 & -2702.683348 & -2702.197168 & -2702.246274 & -2702.244099 \\
\hline BP 4 & -2702.676574 & -2702.190384 & -2702.240576 & -2702.238083 \\
\hline BP5 & -2702.671795 & -2702.185732 & -2702.235325 & -2702.232927 \\
\hline \multicolumn{5}{|c|}{ Relative Energies } \\
\hline Geometry & $\mathrm{E}$ & $H(r e l)$ & $\mathrm{G}(r e l)$ & G Grimme (rel) \\
\hline $\mathrm{R}$ & $79.1 \%$ & $62.2 \%$ & $41.7 \%$ & $40.8 \%$ \\
\hline BP1 & 2.3 & 1.9 & 1.5 & 1.5 \\
\hline BP2 & 0.0 & 0.0 & 0.0 & 0.0 \\
\hline BP3 & 7.6 & 7.4 & 6.9 & 7.0 \\
\hline BP 4 & 6.4 & 6.3 & 6.0 & 6.0 \\
\hline BP5 & 3.7 & 3.7 & 4.1 & 3.8 \\
\hline \multicolumn{5}{|l|}{ S } \\
\hline BP1 & 2.7 & 2.3 & 2.1 & 2.0 \\
\hline BP2 & 0.9 & 0.6 & 0.4 & 0.4 \\
\hline BP3 & 1.4 & 1.3 & 1.5 & 1.3 \\
\hline BP 4 & 5.6 & 5.6 & 5.1 & 5.1 \\
\hline BP5 & 8.6 & 8.5 & 8.4 & 8.3 \\
\hline
\end{tabular}

Absolute energies (E), enthalpies (H), RRHO free energies (G), and quasi-RRHO free energies (Grimme_G), in hartrees, along with the corresponding relative values in $\mathrm{kcal} / \mathrm{mol}$ and predicted ee's, for catalyst $5 e(\mathrm{R}=\mathrm{Me})$

\begin{tabular}{|c|c|c|c|c|}
\hline Geometry & $\mathrm{E}$ & $\mathrm{H}$ & G & G Grimme \\
\hline $\mathrm{R}$ & $94.3 \%$ & $91.7 \%$ & $74.0 \%$ & $\overline{8} 2.6 \%$ \\
\hline BP1 & -3376.654261 & -3376.157324 & -3376.214997 & -3376.211478 \\
\hline BP2 & -3376.659407 & -3376.162082 & -3376.218088 & -3376.215304 \\
\hline BP3 & -3376.645300 & -3376.148234 & -3376.205169 & -3376.202054 \\
\hline BP 4 & -3376.644028 & -3376.147045 & -3376.204058 & -3376.200854 \\
\hline BP5 & -3376.649964 & -3376.152632 & -3376.209444 & -3376.206363 \\
\hline S & & & & \\
\hline BP1 & -3376.653732 & -3376.156767 & -3376.213991 & -3376.210716 \\
\hline BP2 & -3376.657223 & -3376.160136 & -3376.216909 & -3376.213846 \\
\hline BP3 & -3376.654444 & -3376.157217 & -3376.213717 & -3376.210784 \\
\hline BP 4 & -3376.646777 & -3376.149819 & -3376.206919 & -3376.203743 \\
\hline BP5 & -3376.643772 & -3376.146983 & -3376.204748 & -3376.201342 \\
\hline Relative & Energies & & & \\
\hline Geometry & $\mathrm{E}$ & $H(r e l)$ & $G(r e l)$ & G_Grimme (rel) \\
\hline $\mathrm{R}$ & $94.3 \%$ & $91.7 \%$ & $74.0 \%$ & $82.6 \%$ \\
\hline BP1 & 3.2 & 3.0 & 1.9 & 2.4 \\
\hline BP2 & 0.0 & 0.0 & 0.0 & 0.0 \\
\hline BP3 & 8.9 & 8.7 & 8.1 & 8.3 \\
\hline BP 4 & 9.7 & 9.4 & 8.8 & 9.1 \\
\hline BP5 & 5.9 & 5.9 & 5.4 & 5.6 \\
\hline S & & & & \\
\hline BP1 & 3.6 & 3.3 & 2.6 & 2.9 \\
\hline BP2 & 1.4 & 1.2 & 0.7 & 0.9 \\
\hline BP3 & 3.1 & 3.1 & 2.7 & 2.8 \\
\hline BP 4 & 7.9 & 7.7 & 7.0 & 7.3 \\
\hline BP5 & 9.8 & 9.5 & 8.4 & 8.8 \\
\hline
\end{tabular}

Absolute energies (E), enthalpies (H), RRHO free energies (G), and quasi-RRHO free energies (Grimme_G), in hartrees, along with the corresponding relative values in $\mathrm{kcal} / \mathrm{mol}$ and $\bar{d}$ redicted ee's, for catalyst $5 e$ 


\begin{tabular}{|c|c|c|c|c|}
\hline \multicolumn{2}{|l|}{ Geometry } & $\mathrm{H}$ & G & G_Grimme \\
\hline $\mathrm{R}$ & $96.5 \%$ & $97.2 \%$ & $96.9 \%$ & $\overline{9} 7.2 \%$ \\
\hline BP1 & -3298.042796 & -3297.601732 & -3297.656421 & -3297.653164 \\
\hline BP2 & -3298.047462 & -3297.606305 & -3297.659442 & -3297.656942 \\
\hline $\mathrm{BP} 3$ & -3298.032978 & -3297.592158 & -3297.647036 & -3297.643781 \\
\hline BP 4 & -3298.032544 & -3297.591278 & -3297.646021 & -3297.642844 \\
\hline BP 5 & -3298.038028 & -3297.596813 & -3297.651003 & -3297.648039 \\
\hline S & & & & \\
\hline $\mathrm{BP} 1$ & -3298.042262 & -3297.601081 & -3297.655635 & -3297.652487 \\
\hline BP2 & -3298.044968 & -3297.603643 & -3297.656663 & -3297.654236 \\
\hline BP3 & -3298.042311 & -3297.601438 & -3297.655801 & -3297.652759 \\
\hline $\mathrm{BP} 4$ & -3298.035025 & -3297.594205 & -3297.649199 & -3297.645921 \\
\hline BP 5 & -3298.031204 & -3297.590463 & -3297.645709 & -3297.642346 \\
\hline Relative & Energies & & & \\
\hline Geometry & $\mathrm{E}$ & $\mathrm{H}(\mathrm{rel})$ & G(rel) & G_Grimme (rel) \\
\hline $\mathrm{R}$ & $96.5 \%$ & $97.2 \%$ & $96.9 \%$ & $97.2 \%$ \\
\hline BP1 & 2.9 & 2.9 & 1.9 & 2.4 \\
\hline BP2 & 0.0 & 0.0 & 0.0 & 0.0 \\
\hline BP 3 & 9.1 & 8.9 & 7.8 & 8.3 \\
\hline BP 4 & 9.4 & 9.4 & 8.4 & 8.8 \\
\hline BP 5 & 5.9 & 6.0 & 5.3 & 5.6 \\
\hline S & & & & \\
\hline BP 1 & 3.3 & 3.3 & 2.4 & 2.8 \\
\hline BP2 & 1.6 & 1.7 & 1.7 & 1.7 \\
\hline BP3 & 3.2 & 3.1 & 2.3 & 2.6 \\
\hline $\mathrm{BP} 4$ & 7.8 & 7.6 & 6.4 & 6.9 \\
\hline BP 5 & 10.2 & 9.9 & 8.6 & 9.2 \\
\hline
\end{tabular}

Absolute energies (E), enthalpies (H), RRHO free energies (G), and quasi-RRHO free energies (Grimme_G), in hartrees, along with the corresponding relative values in $\mathrm{kcal} / \mathrm{mol}$ and predicted ee's, for catalyst $5 f$

$\begin{array}{lcccc}\text { Geometry } & \text { E } & \text { H } & \text { G } & \text { G_Grimme } \\ \text { R } & 85.9 \% & 85.3 \% & 77.8 \% & 81.6 \% \\ \text { BP1 } & -2859.872385 & -2859.272730 & -2859.327728 & -2859.324933 \\ \text { BP2 } & -2859.875928 & -2859.276022 & -2859.330331 & -2859.327804 \\ \text { BP3 } & -2859.862608 & -2859.262396 & -2859.316783 & -2859.314120 \\ \text { BP4 } & -2859.866223 & -2859.266337 & -2859.321258 & -2859.318619 \\ \text { BP5 } & -2859.870349 & -2859.270650 & -2859.325257 & -2859.322557 \\ \text { S } & & & & \\ \text { BP1 } & -2859.871544 & -2859.271782 & -2859.327251 & -2859.324304 \\ \text { BP2 } & -2859.873927 & -2859.274328 & -2859.329008 & -2859.326343 \\ \text { BP3 } & -2859.873880 & -2859.273387 & -2859.326601 & -2859.324512 \\ \text { BP4 } & -2859.868694 & -2859.268871 & -2859.323994 & -2859.321136 \\ \text { BP5 } & -2859.861018 & -2859.261507 & -2859.316496 & -2859.313696\end{array}$

Relative Energies

$\begin{array}{lc}\text { Geometry } & E \\ \text { R } & 85.9 \% \\ \text { BP1 } & 2.2 \\ \text { BP2 } & 0.0 \\ \text { BP3 } & 8.4 \\ \text { BP4 } & 6.1 \\ \text { BP5 } & 3.5 \\ \text { S } & \end{array}$

$\begin{array}{clc}\text { H }(r e l) & \text { G }(r e l) & \text { G_Grimme }(r e l) \\ 85.3 \% & 77.8 \% & 81.6 \% \\ 2.1 & 1.6 & 1.8 \\ 0.0 & 0.0 & 0.0 \\ 8.6 & 8.5 & 8.6 \\ 6.1 & 5.7 & 5.8 \\ 3.4 & 3.2 & 3.3 \\ 2.7 & 1.9 & 2.2\end{array}$




$\begin{array}{lllll}\text { BP2 } & 1.3 & 1.1 & 0.8 & 0.9 \\ \text { BP3 } & 1.3 & 1.7 & 2.3 & 2.1 \\ \text { BP4 } & 4.5 & 4.5 & 4.0 & 4.2 \\ \text { BP5 } & 9.4 & 9.1 & 8.7 & 8.9\end{array}$

Absolute energies (E), enthalpies (H), RRHO free energies (G), and quasi-RRHO free energies (Grimme G), in hartrees, along with the corresponding relative values in $\mathrm{kcal} / \mathrm{mol}$ and predicted ee's, for catalyst $5 \mathrm{~g}$

$\begin{array}{lcccc}\text { Geometry } & \text { E } & \text { H } & \text { G } & \text { G_Grimme } \\ \text { R } & 46.6 \% & 50.5 \% & 50.5 \% & 54.3 \% \\ \text { BP1 } & -2938.437014 & -2937.781942 & -2937.837529 & -2937.834987 \\ \text { BP2 } & -2938.441686 & -2937.786779 & -2937.841654 & -2937.839413 \\ \text { BP3 } & -2938.429461 & -2937.774236 & -2937.829510 & -2937.826937 \\ \text { BP4 } & -2938.427624 & -2937.772403 & -2937.827653 & -2937.825161 \\ \text { BP5 } & -2938.432982 & -2937.777573 & -2937.832166 & -2937.829932 \\ \text { S } & & & & \\ \text { BP1 } & -2938.436506 & -2937.781477 & -2937.837188 & -2937.834590 \\ \text { BP2 } & -2938.441062 & -2937.786092 & -2937.840967 & -2937.838661 \\ \text { BP3 } & -2938.436971 & -2937.781761 & -2937.836421 & -2937.834295 \\ \text { BP4 } & -2938.432805 & -2937.777362 & -2937.832681 & -2937.830125 \\ \text { BP5 } & -2938.424834 & -2937.770272 & -2937.825256 & -2937.822943\end{array}$

Relative Energies

\begin{tabular}{|c|c|c|c|c|}
\hline Geometry & $\mathrm{E}$ & $\mathrm{H}(r \in l)$ & $G(r e l)$ & G_Grimme(rel) \\
\hline $\mathrm{R}$ & $46.6 \%$ & $50.5 \%$ & $50.5 \%$ & $54.3 \%$ \\
\hline BP1 & 2.9 & 3.0 & 2.6 & 2.8 \\
\hline BP2 & 0.0 & 0.0 & 0.0 & 0.0 \\
\hline BP3 & 7.7 & 7.9 & 7.6 & 7.8 \\
\hline BP 4 & 8.8 & 9.0 & 8.8 & 8.9 \\
\hline BP5 & 5.5 & 5.8 & 6.0 & 5.9 \\
\hline \multicolumn{5}{|l|}{$\mathrm{S}$} \\
\hline BP1 & 3.3 & 3.3 & 2.8 & 3.0 \\
\hline BP2 & 0.4 & 0.4 & 0.4 & 0.5 \\
\hline BP3 & 3.0 & 3.1 & 3.3 & 3.2 \\
\hline BP 4 & 5.6 & 5.9 & 5.6 & 5.8 \\
\hline BP5 & 10.6 & 10.4 & 10.3 & 10.3 \\
\hline
\end{tabular}

Absolute energies (E), enthalpies (H), RRHO free energies (G), and quasi-RRHO free energies (Grimme_G), in hartrees, along with the corresponding relative values in $\mathrm{kcal} / \mathrm{mol}$ and predicted ee's, for catalyst $5 \mathrm{~h}$

$\begin{array}{lcccc}\text { Geometry } & \text { E } & \text { H } & \text { G } & \text { G_Grimme } \\ \text { R } & 92.4 \% & 89.6 \% & 80.9 \% & 85.3 \% \\ \text { BP1 } & -2776.282689 & -2775.834228 & -2775.885342 & -2775.882873 \\ \text { BP2 } & -2776.285616 & -2775.837085 & -2775.888219 & -2775.885660 \\ \text { BP3 } & -2776.273891 & -2775.825620 & -2775.876990 & -2775.874416 \\ \text { BP4 } & -2776.277381 & -2775.828804 & -2775.879644 & -2775.877282 \\ \text { BP5 } & -2776.279493 & -2775.831232 & -2775.882140 & -2775.879722 \\ \text { S } & & & & \\ \text { BP1 } & -2776.282932 & -2775.834441 & -2775.885351 & -2775.882934 \\ \text { BP2 } & -2776.283130 & -2775.834988 & -2775.886707 & -2775.883904 \\ \text { BP3 } & -2776.282709 & -2775.834122 & -2775.885407 & -2775.882820 \\ \text { BP4 } & -2776.275872 & -2775.827470 & -2775.878781 & -2775.876201 \\ \text { BP5 } & -2776.273631 & -2775.825034 & -2775.876090 & -2775.873616\end{array}$

Relative Energies 


$\begin{array}{lcccc}\text { Geometry } & E & H(r e l) & G(r e l) & \text { G_Grimme }(r e l) \\ \text { R } & 92.4 \% & 89.6 \% & 80.9 \% & 85.3 \% \\ \text { BP1 } & 1.8 & 1.8 & 1.8 & 1.7 \\ \text { BP2 } & 0.0 & 0.0 & 0.0 & 0.0 \\ \text { BP3 } & 7.4 & 7.2 & 7.0 & 7.1 \\ \text { BP4 } & 5.2 & 5.2 & 5.4 & 5.3 \\ \text { BP5 } & 3.8 & 3.7 & 3.8 & 3.7 \\ \text { S } & & 1.7 & 1.8 & 1.7 \\ \text { BP1 } & 1.7 & 1.3 & 0.9 & 1.1 \\ \text { BP2 } & 1.6 & 1.9 & 1.8 & 1.8 \\ \text { BP3 } & 1.8 & 6.0 & 5.9 & 5.9 \\ \text { BP4 } & 6.1 & 7.6 & 7.6 & 7.6 \\ \text { BP5 } & 7.5 & & & \end{array}$

Absolute energies (E), enthalpies (H), RRHO free energies (G), and quasi-RRHO free energies (Grimme_G), in hartrees, along with the corresponding relative values in $\mathrm{kcal} / \mathrm{mol}$ and predicted ee's, for catalyst $5 i$ ( $R=M e)$

\begin{tabular}{|c|c|c|c|c|}
\hline Geometry & $\mathrm{E}$ & $\mathrm{H}$ & G & G Grimme \\
\hline $\mathrm{R}$ & $95.8 \%$ & $94.0 \%$ & $80.3 \%$ & $88.3 \%$ \\
\hline BP1 & -2887.065102 & -2886.581102 & -2886.634332 & -2886.631819 \\
\hline BP2 & -2887.069046 & -2886.584847 & -2886.637490 & -2886.635215 \\
\hline BP3 & -2887.056008 & -2886.572182 & -2886.625590 & -2886.623113 \\
\hline BP 4 & -2887.056818 & -2886.572898 & -2886.626631 & -2886.623922 \\
\hline BP5 & -2887.061907 & -2886.577863 & -2886.631102 & -2886.628584 \\
\hline \multicolumn{5}{|l|}{$\mathrm{S}$} \\
\hline BP1 & -2887.065141 & -2886.581397 & -2886.635131 & -2886.632399 \\
\hline BP2 & -2887.066526 & -2886.582580 & -2886.635980 & -2886.633369 \\
\hline BP3 & -2887.065383 & -2886.580944 & -2886.633382 & -2886.631149 \\
\hline BP 4 & -2887.057392 & -2886.573455 & -2886.626889 & -2886.624229 \\
\hline BP 5 & -2887.057053 & -2886.573273 & -2886.627195 & -2886.624319 \\
\hline \multicolumn{5}{|c|}{ Relative Energies } \\
\hline Geometry & $E$ & $\mathrm{H}(\mathrm{rel})$ & $\mathrm{G}(r e l)$ & G Grimme (rel) \\
\hline $\mathrm{R}$ & $95.8 \%$ & $94.0 \%$ & $80.3 \%$ & $88.3 \%$ \\
\hline BP1 & 2.5 & 2.4 & 2.0 & 2.1 \\
\hline BP2 & 0.0 & 0.0 & 0.0 & 0.0 \\
\hline BP3 & 8.2 & 7.9 & 7.5 & 7.6 \\
\hline BP 4 & 7.7 & 7.5 & 6.8 & 7.1 \\
\hline BP5 & 4.5 & 4.4 & 4.0 & 4.2 \\
\hline \multicolumn{5}{|l|}{$\mathrm{S}$} \\
\hline BP1 & 2.5 & 2.2 & 1.5 & 1.8 \\
\hline BP2 & 1.6 & 1.4 & 0.9 & 1.2 \\
\hline BP3 & 2.3 & 2.4 & 2.6 & 2.6 \\
\hline BP 4 & 7.3 & 7.1 & 6.7 & 6.9 \\
\hline BP 5 & 7 & 7.3 & 6.5 & 6.8 \\
\hline
\end{tabular}

Absolute energies (E), enthalpies (H), RRHO free energies (G), and quasi-RRHO free energies (Grimme_G), in hartrees, along with the corresponding relative values in $\mathrm{kcal} / \mathrm{mol}$ and predicted ee's, for catalyst $5 i$

$\begin{array}{lcccc}\text { Geometry } & \text { E } & \text { H } & \text { G } & \text { G Grimme } \\ \text { R } & 95.7 \% & 94.6 \% & 93.9 \% & 93.9 \% \\ \text { BP1 } & -2808.453659 & -2808.025923 & -2808.076913 & -2808.074378 \\ \text { BP2 } & -2808.456869 & -2808.028903 & -2808.079720 & -2808.077191 \\ \text { BP3 } & -2808.444070 & -2808.016436 & -2808.067699 & -2808.065076 \\ \text { BP4 } & -2808.445639 & -2808.017802 & -2808.068749 & -2808.066269\end{array}$




\begin{tabular}{|c|c|c|c|c|}
\hline $\begin{array}{l}\text { BP5 } \\
\text { S }\end{array}$ & -2808.450315 & -2808.022525 & -2808.072951 & -2808.070650 \\
\hline $\mathrm{BP} 1$ & -2808.453887 & -2808.025982 & -2808.076768 & -2808.074308 \\
\hline BP2 & -2808.454022 & -2808.026355 & -2808.077381 & -2808.074801 \\
\hline $\mathrm{BP} 3$ & -2808.453450 & -2808.025265 & -2808.075330 & -2808.073178 \\
\hline BP 4 & -2808.445997 & -2808.018143 & -2808.069134 & -2808.066593 \\
\hline BP 5 & -2808.445537 & -2808.017804 & -2808.069395 & -2808.066569 \\
\hline \multicolumn{5}{|c|}{ Relative Energies } \\
\hline Geometry & $\mathrm{E}$ & $\mathrm{H}(r \in l)$ & $G(r e l)$ & G_Grimme (rel) \\
\hline $\mathrm{R}$ & $95.7 \%$ & $94.6 \%$ & $93.9 \%$ & $93.9 \%$ \\
\hline BP1 & 2.0 & 1.9 & 1.8 & 1.8 \\
\hline BP2 & 0.0 & 0.0 & 0.0 & 0.0 \\
\hline $\mathrm{BP} 3$ & 8.0 & 7.8 & 7.5 & 7.6 \\
\hline BP 4 & 7.0 & 7.0 & 6.9 & 6.9 \\
\hline BP 5 & 4.1 & 4.0 & 4.2 & 4.1 \\
\hline \multicolumn{5}{|l|}{ S } \\
\hline BP1 & 1.9 & 1.8 & 1.9 & 1.8 \\
\hline BP2 & 1.8 & 1.6 & 1.5 & 1.5 \\
\hline BP3 & 2.1 & 2.3 & 2.8 & 2.5 \\
\hline BP 4 & 6.8 & 6.8 & 6.6 & 6.7 \\
\hline BP 5 & 7.1 & 7.0 & 6.5 & 6.7 \\
\hline
\end{tabular}

Absolute energies (E), enthalpies (H), RRHO free energies (G), and quasi-RRHO free energies (Grimme_G), in hartrees, along with the corresponding relative values in $\mathrm{kcal} / \mathrm{mol}$ and predicted ee's, for catalyst $5 j$

$\begin{array}{lcccc}\text { Geometry } & \text { E } & \text { H } & \text { G } & \text { G_Grimme } \\ \text { R } & 45.4 \% & 47.0 \% & 61.8 \% & 51.9 \% \\ \text { BP1 } & -3085.977678 & -3085.386111 & -3085.442788 & -3085.439325 \\ \text { BP2 } & -3085.980090 & -3085.388442 & -3085.445049 & -3085.441543 \\ \text { BP3 } & -3085.967032 & -3085.375621 & -3085.431914 & -3085.428599 \\ \text { BP4 } & -3085.970579 & -3085.378825 & -3085.435406 & -3085.432017 \\ \text { BP5 } & -3085.971397 & -3085.379691 & -3085.435623 & -3085.432460 \\ \text { S } & & & & \\ \text { BP1 } & -3085.979490 & -3085.387819 & -3085.444167 & -3085.440843 \\ \text { BP2 } & -3085.975838 & -3085.384297 & -3085.441060 & -3085.437528 \\ \text { BP3 } & -3085.976596 & -3085.384803 & -3085.440735 & -3085.437596 \\ \text { BP4 } & -3085.968966 & -3085.377508 & -3085.434245 & -3085.430771 \\ \text { BP5 } & -3085.968422 & -3085.376801 & -3085.432962 & -3085.429765\end{array}$

Relative Energies

$\begin{array}{lcccc}\text { Geometry } & E & \text { H (rel) } & \text { G(rel) } & \text { G_Grimme }(r e l) \\ \text { R } & 45.4 \% & 47.0 \% & 61.8 \% & 51.9 \% \\ \text { BP1 } & 1.5 & 1.5 & 1.4 & 1.4 \\ \text { BP2 } & 0.0 & 0.0 & 0.0 & 0.0 \\ \text { BP3 } & 8.2 & 8.0 & 8.2 & 8.1 \\ \text { BP4 } & 6.0 & 6.0 & 6.1 & 6.0 \\ \text { BP5 } & 5.5 & 5.5 & 5.9 & 5.7 \\ \text { S } & & & & 0.4 \\ \text { BP1 } & 0.4 & 0.4 & 0.6 & 2.5 \\ \text { BP2 } & 2.7 & 2.6 & 2.5 & 2.5 \\ \text { BP3 } & 2.2 & 2.3 & 2.7 & 6.8 \\ \text { BP4 } & 7.0 & 6.9 & 6.8 & 7.4 \\ \text { BP5 } & 7.3 & 7.3 & 7.6 & \end{array}$




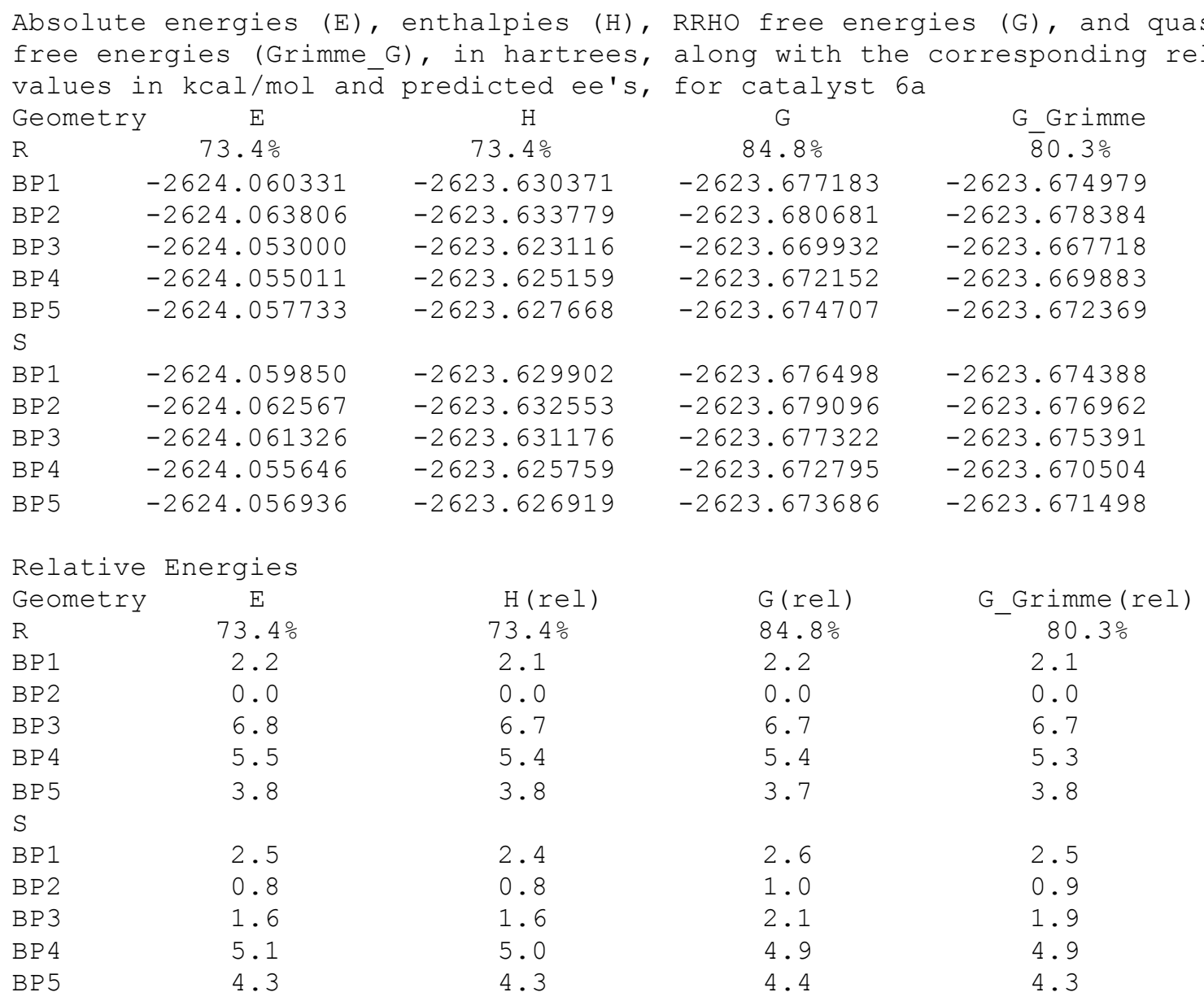

Absolute energies (E), enthalpies (H), RRHO free energies (G), and quasi-RRHO free energies (Grimme G), in hartrees, along with the corresponding relative values in $\mathrm{kcal} / \mathrm{mol}$ and predicted ee's, for catalyst 6b

$\begin{array}{lcccc}\text { Geometry } & \text { E } & \text { H } & \text { G } & \text { G_Grimme } \\ \text { R } & 89.4 \% & 90.4 \% & 92.5 \% & 91.6 \% \\ \text { BP1 } & -2822.503230 & -2822.089095 & -2822.137512 & -2822.135282 \\ \text { BP2 } & -2822.506817 & -2822.092499 & -2822.140694 & -2822.138528 \\ \text { BP3 } & -2822.495313 & -2822.081163 & -2822.129737 & -2822.127442 \\ \text { BP4 } & -2822.497287 & -2822.083037 & -2822.131618 & -2822.129331 \\ \text { BP5 } & -2822.500771 & -2822.086568 & -2822.135012 & -2822.132744 \\ \text { S } & & & & \\ \text { BP1 } & -2822.503159 & -2822.088929 & -2822.137209 & -2822.135027 \\ \text { BP2 } & -2822.504830 & -2822.090399 & -2822.138399 & -2822.136313 \\ \text { BP3 } & -2822.504150 & -2822.089877 & -2822.137939 & -2822.135839 \\ \text { BP4 } & -2822.497098 & -2822.083043 & -2822.132203 & -2822.129662 \\ \text { BP5 } & -2822.498946 & -2822.084511 & -2822.132511 & -2822.130468\end{array}$

Relative Energies

$\begin{array}{lcccc}\text { Geometry } & E & \text { H }(r e l) & \text { G(rel) } & \text { G_Grimme }(r e l) \\ \text { R } & 89.4 \% & 90.4 \% & 92.5 \% & 91.6 \% \\ \text { BP1 } & 2.3 & 2.1 & 2.0 & 2.0 \\ \text { BP2 } & 0.0 & 0.0 & 0.0 & 0.0 \\ \text { BP3 } & 7.2 & 7.1 & 6.9 & 7.0 \\ \text { BP4 } & 6.0 & 5.9 & 5.7 & 5.8\end{array}$




$\begin{array}{lllll}\text { BP5 } & 3.8 & 3.7 & 3.6 & 3.6 \\ \text { S } & & & & \\ \text { BP1 } & 2.3 & 2.2 & 2.2 & 2.2 \\ \text { BP2 } & 1.2 & 1.3 & 1.4 & 1.4 \\ \text { BP3 } & 1.7 & 1.6 & 1.7 & 1.7 \\ \text { BP4 } & 6.1 & 5.9 & 5.3 & 5.6 \\ \text { BP5 } & 4.9 & 5.0 & 5.1 & 5.1\end{array}$

Absolute energies (E), enthalpies (H), RRHO free energies (G), and quasi-RRHO free energies (Grimme_G), in hartrees, along with the corresponding relative values in $\mathrm{kcal} / \mathrm{mol}$ and predicted ee's, for catalyst $6 \mathrm{c}$

\begin{tabular}{|c|c|c|c|c|}
\hline Geometry & $\mathrm{E}$ & $\mathrm{H}$ & G & G Grimme \\
\hline $\mathrm{R}$ & $91.0 \%$ & $91.4 \%$ & $93.7 \%$ & $\overline{9} 2.9 \%$ \\
\hline BP1 & -3543.302996 & -3542.891012 & -3542.940408 & -3542.938132 \\
\hline BP2 & -3543.306211 & -3542.894391 & -3542.944137 & -3542.941679 \\
\hline BP3 & -3543.294347 & -3542.882913 & -3542.932990 & -3542.930469 \\
\hline BP 4 & -3543.296763 & -3542.885108 & -3542.935060 & -3542.932564 \\
\hline BP5 & -3543.300093 & -3542.888421 & -3542.937776 & -3542.935550 \\
\hline $\mathrm{S}$ & & & & \\
\hline BP1 & -3543.302617 & -3542.890955 & -3542.940780 & -3542.938290 \\
\hline BP2 & -3543.304163 & -3542.892322 & -3542.941891 & -3542.939500 \\
\hline BP3 & -3543.303255 & -3542.891256 & -3542.940298 & -3542.938150 \\
\hline BP 4 & -3543.296945 & -3542.885016 & -3542.934535 & -3542.932210 \\
\hline BP5 & -3543.294082 & -3542.882718 & -3542.933034 & -3542.930442 \\
\hline Relative & Energies & & & \\
\hline Geometry & $E$ & $H(r e l)$ & $G(r e l)$ & G Grimme(rel) \\
\hline $\mathrm{R}$ & $91.0 \%$ & $91.4 \%$ & $93.7 \%$ & $92.9 \%$ \\
\hline BP1 & 2.0 & 2.1 & 2.3 & 2.2 \\
\hline BP2 & 0.0 & 0.0 & 0.0 & 0.0 \\
\hline BP3 & 7.4 & 7.2 & 7.0 & 7.0 \\
\hline BP 4 & 5.9 & 5.8 & 5.7 & 5.7 \\
\hline BP5 & 3.8 & 3.7 & 4.0 & 3.8 \\
\hline $\mathrm{S}$ & & & & \\
\hline BP1 & 2.3 & 2.2 & 2.1 & 2.1 \\
\hline BP2 & 1.3 & 1.3 & 1.4 & 1.4 \\
\hline BP3 & 1.9 & 2.0 & 2.4 & 2.2 \\
\hline BP 4 & 5.8 & 5.9 & 6.0 & 5.9 \\
\hline BP 5 & 7.6 & 7.3 & 7.0 & 7.1 \\
\hline
\end{tabular}

Absolute energies (E), enthalpies (H), RRHO free energies (G), and quasi-RRHO free energies (Grimme_G), in hartrees, along with the corresponding relative values in $\mathrm{kcal} / \mathrm{mol}$ and predicted ee's, for catalyst $6 \mathrm{~d}$

$\begin{array}{lcccc}\text { Geometry } & \mathrm{E} & \mathrm{H} & \mathrm{G} & \text { G Grimme } \\ \text { R } & 57.9 \% & 70.0 \% & 80.7 \% & 80.1 \% \\ \text { BP1 } & -2702.679531 & -2702.194061 & -2702.243277 & -2702.241055 \\ \text { BP2 } & -2702.682106 & -2702.196736 & -2702.245970 & -2702.243854 \\ \text { BP3 } & -2702.670765 & -2702.185496 & -2702.235158 & -2702.232814 \\ \text { BP4 } & -2702.673628 & -2702.188094 & -2702.236975 & -2702.234902 \\ \text { BP5 } & -2702.676618 & -2702.191067 & -2702.239754 & -2702.237831 \\ \text { S } & & & & \\ \text { BP1 } & -2702.678702 & -2702.193208 & -2702.242130 & -2702.240055 \\ \text { BP2 } & -2702.681216 & -2702.195596 & -2702.244541 & -2702.242424 \\ \text { BP3 } & -2702.679941 & -2702.194240 & -2702.242946 & -2702.241049 \\ \text { BP4 } & -2702.674866 & -2702.189305 & -2702.238445 & -2702.236317\end{array}$




\begin{tabular}{|c|c|c|c|c|}
\hline BP 5 & -2702.669546 & -2702.183691 & -2702.232485 & -2702.230415 \\
\hline \multicolumn{5}{|c|}{ Relative Energies } \\
\hline Geometry & $E$ & $\mathrm{H}(\mathrm{rel})$ & $G(r e l)$ & G_Grimme (rel) \\
\hline $\mathrm{R}$ & $57.9 \%$ & $70.0 \%$ & $80.7 \%$ & $-80.1 \%$ \\
\hline BP1 & 1.6 & 1.7 & 1.7 & 1.8 \\
\hline BP2 & 0.0 & 0.0 & 0.0 & 0.0 \\
\hline $\mathrm{BP} 3$ & 7.1 & 7.1 & 6.8 & 6.9 \\
\hline BP 4 & 5.3 & 5.4 & 5.6 & 5.6 \\
\hline BP 5 & 3.4 & 3.6 & 3.9 & 3.8 \\
\hline \multicolumn{5}{|l|}{ S } \\
\hline BP1 & 2.1 & 2.2 & 2.4 & 2.4 \\
\hline BP2 & 0.6 & 0.7 & 0.9 & 0.9 \\
\hline BP 3 & 1.4 & 1.6 & 1.9 & 1.8 \\
\hline $\mathrm{BP} 4$ & 4.5 & 4.7 & 4.7 & 4.7 \\
\hline BP 5 & 7.9 & 8.2 & 8.5 & 8.4 \\
\hline
\end{tabular}

Absolute energies (E), enthalpies (H), RRHO free energies (G), and quasi-RRHO free energies (Grimme_G), in hartrees, along with the corresponding relative values in kcal/mol and predicted ee's, for catalyst $6 e$

\begin{tabular}{lcccc} 
Geometry & $\mathrm{E}$ & $\mathrm{H}$ & $\mathrm{G}$ & \multicolumn{1}{c}{ Grimme } \\
R & $96.0 \%$ & $94.7 \%$ & $96.1 \%$ & $95.1 \%$ \\
BP1 & -3298.058081 & -3297.617170 & -3297.671873 & -3297.668809 \\
BP2 & -3298.060876 & -3297.619798 & -3297.674633 & -3297.671416 \\
BP3 & -3298.047935 & -3297.607202 & -3297.661582 & -3297.658610 \\
BP4 & -3298.051842 & -3297.611092 & -3297.665950 & -3297.662879 \\
BP5 & -3298.053618 & -3297.612540 & -3297.666896 & -3297.663975 \\
S & & & & \\
BP1 & -3298.057810 & -3297.616959 & -3297.671727 & -3297.668604 \\
BP2 & -3298.058216 & -3297.617281 & -3297.671849 & -3297.668809 \\
BP3 & -3298.056009 & -3297.614880 & -3297.669103 & -3297.666205 \\
BP4 & -3298.052134 & -3297.611218 & -3297.665655 & -3297.662730 \\
BP5 & -3298.045020 & -3297.604107 & -3297.659121 & -3297.655931
\end{tabular}

$\begin{array}{lcccc}\begin{array}{l}\text { Relative } \\ \text { Geometry }\end{array} & \text { Energies } & \text { H (rel) } & \text { G(rel) } & \text { G_Grimme }(r e 1) \\ \text { R } & 96.0 \% & 94.7 \% & 96.1 \% & 95.1 \% \\ \text { BP1 } & 1.8 & 1.6 & 1.7 & 1.6 \\ \text { BP2 } & 0.0 & 0.0 & 0.0 & 0.0 \\ \text { BP3 } & 8.1 & 7.9 & 8.2 & 8.0 \\ \text { BP4 } & 5.7 & 5.5 & 5.4 & 5.4 \\ \text { BP5 } & 4.6 & 4.6 & 4.9 & 4.7 \\ \text { S } & & & & 1.8 \\ \text { BP1 } & 1.9 & 1.8 & 1.8 & 1.6 \\ \text { BP2 } & 1.7 & 1.6 & 1.7 & 3.3 \\ \text { BP3 } & 3.1 & 3.1 & 3.5 & 5.5 \\ \text { BP4 } & 5.5 & 5.4 & 5.6 & 9.7 \\ \text { BP5 } & 9.9 & 9.8 & 9.7 & \end{array}$

Absolute energies (E), enthalpies (H), RRHO free energies (G), and quasi-RRHO free energies (Grimme_G), in hartrees, along with the corresponding relative values in kcal/mol and predicted ee's, for catalyst $6 \mathrm{f}$
$\begin{array}{ll}\text { Geometry } & \mathrm{E} \\ \mathrm{R} & 63.1 \%\end{array}$
BP 1
$\mathrm{H}$
$76.8 \%$
$-2859.281997$
$91.6 \%$
$-2859.336708$

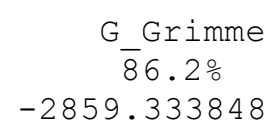




$\begin{array}{lcccc}\text { BP2 } & -2859.884035 & -2859.284930 & -2859.339393 & -2859.336587 \\ \text { BP3 } & -2859.873452 & -2859.274364 & -2859.328152 & -2859.325776 \\ \text { BP4 } & -2859.875919 & -2859.276576 & -2859.330446 & -2859.327997 \\ \text { BP5 } & -2859.880158 & -2859.280942 & -2859.334719 & -2859.332249 \\ \text { S } & & & & \\ \text { BP1 } & -2859.879377 & -2859.280453 & -2859.335142 & -2859.332298 \\ \text { BP2 } & -2859.882986 & -2859.283478 & -2859.337239 & -2859.334756 \\ \text { BP3 } & -2859.882116 & -2859.282885 & -2859.336705 & -2859.334244 \\ \text { BP4 } & -2859.876804 & -2859.277668 & -2859.331979 & -2859.329296 \\ \text { BP5 } & -2859.870172 & -2859.271212 & -2859.326089 & -2859.323186 \\ & & & & \\ \text { Relative Energies } & \text { E } & \text { H(rel) } & \text { G(rel) } & \text { G_Grimme (rel) } \\ \text { Geometry } & 63.1 \% & 76.8 \% & 91.6 \% & 86.2 \% \\ \text { R } & 2.0 & 1.8 & 1.7 & 1.7 \\ \text { BP1 } & 0.0 & 0.0 & 0.0 & 6.0 \\ \text { BP2 } & 6.6 & 6.6 & 7.1 & 5.4 \\ \text { BP3 } & 5.1 & 5.2 & 5.6 & 2.7 \\ \text { BP4 } & 2.4 & 2.5 & 2.9 & 2.7 \\ \text { BP5 } & 2.9 & 2.8 & 2.7 & 1.1 \\ \text { S } & 0.7 & 0.9 & 1.4 & 1.5 \\ \text { BP1 } & 1.2 & 1.3 & 1.7 & 4.6 \\ \text { BP2 } & 4.5 & 8.6 & 8.3 & 8.4 \\ \text { BP3 } & 8.7 & & & \end{array}$

Absolute energies (E), enthalpies (H), RRHO free energies (G), and quasi-RRHO free energies (Grimme_G), in hartrees, along with the corresponding relative values in $\mathrm{kcal} / \mathrm{mol}$ and predicted ee's, for catalyst $6 \mathrm{~g}$

\begin{tabular}{|c|c|c|c|c|}
\hline Geometry & $\mathrm{E}$ & $\mathrm{H}$ & G & G Grimme \\
\hline $\mathrm{R}$ & $-30.6 \%$ & $-10.3 \%$ & $60.6 \%$ & $-34.9 \%$ \\
\hline BP1 & -2938.458430 & -2937.803909 & -2937.860340 & -2937.857354 \\
\hline BP2 & -2938.459556 & -2937.804731 & -2937.861145 & -2937.858053 \\
\hline BP3 & -2938.447967 & -2937.793264 & -2937.849701 & -2937.846606 \\
\hline BP 4 & -2938.444938 & -2937.789595 & -2937.846463 & -2937.843088 \\
\hline BP5 & -2938.453856 & -2937.799121 & -2937.854433 & -2937.851908 \\
\hline S & & & & \\
\hline BP1 & -2938.456595 & -2937.801313 & -2937.857136 & -2937.854352 \\
\hline BP2 & -2938.460037 & -2937.805001 & -2937.860422 & -2937.857772 \\
\hline BP3 & -2938.454932 & -2937.800287 & -2937.856702 & -2937.853732 \\
\hline BP 4 & -2938.453793 & -2937.798833 & -2937.854605 & -2937.851877 \\
\hline BP5 & -2938.442850 & -2937.788642 & -2937.844899 & -2937.841940 \\
\hline Relative & Energies & & & \\
\hline Geometry & $\mathrm{E}$ & $H(r e l)$ & $G(r e l)$ & G_Grimme (rel) \\
\hline $\mathrm{R}$ & $-30.6 \%$ & $-10.3 \div$ & $60.6 \%$ & $34.9 \%$ \\
\hline BP1 & 1.0 & 0.7 & 0.5 & 0.4 \\
\hline BP2 & 0.3 & 0.2 & 0.0 & 0.0 \\
\hline BP3 & 7.6 & 7.4 & 7.2 & 7.2 \\
\hline BP 4 & 9.5 & 9.7 & 9.2 & 9.4 \\
\hline BP5 & 3.9 & 3.7 & 4.2 & 3.9 \\
\hline S & & & & \\
\hline BP1 & 2.2 & 2.3 & 2.5 & 2.3 \\
\hline BP2 & 0.0 & 0.0 & 0.5 & 0.2 \\
\hline BP3 & 3.2 & 3.0 & 2.8 & 2.7 \\
\hline BP 4 & 3.9 & 3.9 & 4.1 & 3.9 \\
\hline
\end{tabular}


Absolute energies (E), enthalpies (H), RRHO free energies (G), and quasi-RRHO free energies (Grimme_G), in hartrees, along with the corresponding relative values in $\mathrm{kcal} / \mathrm{mol}$ and predicted ee's, for catalyst $6 \mathrm{~h}$

\begin{tabular}{|c|c|c|c|c|}
\hline Geometry & $\mathrm{E}$ & $\mathrm{H}$ & G & G Grimme \\
\hline $\mathrm{R}$ & $90.5 \%$ & $90.4 \%$ & $90.1 \%$ & $\overline{9} 0.5 \%$ \\
\hline BP1 & -2776.277257 & -2775.828808 & -2775.879714 & -2775.877312 \\
\hline BP2 & -2776.279976 & -2775.831603 & -2775.882593 & -2775.880129 \\
\hline BP 3 & -2776.268620 & -2775.820456 & -2775.871885 & -2775.869307 \\
\hline $\mathrm{BP} 4$ & -2776.272062 & -2775.823579 & -2775.874497 & -2775.872076 \\
\hline BP 5 & -2776.274420 & -2775.826298 & -2775.876807 & -2775.874581 \\
\hline S & & & & \\
\hline BP1 & -2776.277421 & -2775.829116 & -2775.880178 & -2775.877654 \\
\hline BP2 2 & -2776.277779 & -2775.829419 & -2775.880440 & -2775.877951 \\
\hline BP3 & -2776.276808 & -2775.828216 & -2775.878696 & -2775.876460 \\
\hline BP 4 & -2776.270506 & -2775.821844 & -2775.872306 & -2775.870098 \\
\hline BP 5 & -2776.269233 & -2775.820999 & -2775.872074 & -2775.869588 \\
\hline Relative & Energies & & & \\
\hline Geometry & $\mathrm{E}$ & $\mathrm{H}(r e l)$ & $\mathrm{G}(\mathrm{rel})$ & G_Grimme (rel) \\
\hline $\mathrm{R}$ & $90.5 \%$ & $90.4 \%$ & $90.1 \%$ & $90.5 \%$ \\
\hline BP1 & 1.7 & 1.8 & 1.8 & 1.8 \\
\hline BP2 & 0.0 & 0.0 & 0.0 & 0.0 \\
\hline BP3 & 7.1 & 7.0 & 6.7 & 6.8 \\
\hline BP 4 & 5.0 & 5.0 & 5.1 & 5.1 \\
\hline BP 5 & 3.5 & 3.3 & 3.6 & 3.5 \\
\hline S & & & & \\
\hline BP1 & 1.6 & 1.6 & 1.5 & 1.6 \\
\hline BP2 & 1.4 & 1.4 & 1.4 & 1.4 \\
\hline BP 3 & 2.0 & 2.1 & 2.4 & 2.3 \\
\hline BP 4 & 5.9 & 6.1 & 6.5 & 6.3 \\
\hline BP5 & 6.7 & 6.7 & 6.6 & 6.6 \\
\hline
\end{tabular}

Absolute energies (E), enthalpies (H), RRHO free energies (G), and quasi-RRHO free energies (Grimme_G), in hartrees, along with the corresponding relative values in $\mathrm{kcal} / \mathrm{mol}$ and predicted ee's, for catalyst $6 i$

$\begin{array}{lcccc}\text { Geometry } & \text { E } & \text { H } & \text { G } & \text { G_Grimme } \\ \text { R } & 93.6 \% & 89.2 \% & 75.9 \% & 83.0 \% \\ \text { BP1 } & -2808.452770 & -2808.024793 & -2808.075666 & -2808.073158 \\ \text { BP2 } & -2808.455756 & -2808.027418 & -2808.077562 & -2808.075339 \\ \text { BP3 } & -2808.443373 & -2808.015689 & -2808.067074 & -2808.064384 \\ \text { BP4 } & -2808.444966 & -2808.017154 & -2808.068396 & -2808.065752 \\ \text { BP5 } & -2808.449836 & -2808.021972 & -2808.072454 & -2808.070112 \\ \text { S } & & & & \\ \text { BP1 } & -2808.452966 & -2808.025002 & -2808.075670 & -2808.073217 \\ \text { BP2 } & -2808.453343 & -2808.025347 & -2808.076099 & -2808.073605 \\ \text { BP3 } & -2808.452078 & -2808.023829 & -2808.073947 & -2808.071754 \\ \text { BP4 } & -2808.445322 & -2808.017315 & -2808.068345 & -2808.065790 \\ \text { BP5 } & -2808.445600 & -2808.017624 & -2808.068612 & -2808.066037\end{array}$

Relative Energies

$\begin{array}{lc}\text { Geometry } & E \\ \text { R } & 93.6 \% \\ \text { BP1 } & 1.9\end{array}$

$\begin{array}{cl}\mathrm{H}(\mathrm{rel}) & \mathrm{G}(\mathrm{rel}) \\ 89.2 \% & 75.9 \% \\ 1.6 & 1.2\end{array}$

G Grimme (rel) $83.0 \%$ 1.4 


$\begin{array}{lllll}\text { BP2 } & 0.0 & 0.0 & 0.0 & 0.0 \\ \text { BP3 } & 7.8 & 7.4 & 6.6 & 6.9 \\ \text { BP4 } & 6.8 & 6.4 & 5.8 & 6.0 \\ \text { BP5 } & 3.7 & 3.4 & 3.2 & 3.3 \\ \text { S } & & & & \\ \text { BP1 } & 1.8 & 1.5 & 1.2 & 1.3 \\ \text { BP2 } & 1.5 & 1.3 & 0.9 & 1.1 \\ \text { BP3 } & 2.3 & 2.3 & 2.3 & 2.2 \\ \text { BP4 } & 6.5 & 6.3 & 5.8 & 6.0 \\ \text { BP5 } & 6.4 & 6.1 & 5.6 & 5.8\end{array}$

Absolute energies (E), enthalpies (H), RRHO free energies (G), and quasi-RRHO free energies (Grimme G), in hartrees, along with the corresponding relative values in $\mathrm{kcal} / \mathrm{mol}$ and predicted ee's, for catalyst $6 j$

$\begin{array}{lcccc}\text { Geometry } & \text { E } & \text { H } & G & \text { G_Grimme } \\ \text { R } & 87.7 \% & 82.7 \% & 62.7 \% & 72.8 \% \\ \text { BP1 } & -3085.974716 & -3085.383307 & -3085.440029 & -3085.436546 \\ \text { BP2 } & -3085.977455 & -3085.385812 & -3085.442074 & -3085.438721 \\ \text { BP3 } & -3085.964878 & -3085.373714 & -3085.430277 & -3085.426827 \\ \text { BP4 } & -3085.967525 & -3085.376189 & -3085.433122 & -3085.429572 \\ \text { BP5 } & -3085.970387 & -3085.378905 & -3085.435323 & -3085.431906 \\ \text { S } & & & & \\ \text { BP1 } & -3085.975696 & -3085.384309 & -3085.440999 & -3085.437510 \\ \text { BP2 } & -3085.973727 & -3085.382469 & -3085.440341 & -3085.436258 \\ \text { BP3 } & -3085.974282 & -3085.382461 & -3085.438253 & -3085.435099 \\ \text { BP4 } & -3085.966943 & -3085.375548 & -3085.432362 & -3085.428848 \\ \text { BP5 } & -3085.967807 & -3085.376452 & -3085.433520 & -3085.429907\end{array}$

$\begin{array}{lcccc}\begin{array}{l}\text { Relative Energies } \\ \text { Geometry }\end{array} & \text { E } & \text { H }(r e l) & \text { G(rel) } & \text { G_Grimme }(r e l) \\ \text { R } & 87.7 \% & 82.7 \% & 62.7 \% & 72.8 \% \\ \text { BP1 } & 1.7 & 1.6 & 1.3 & 1.4 \\ \text { BP2 } & 0.0 & 0.0 & 0.0 & 0.0 \\ \text { BP3 } & 7.9 & 7.6 & 7.4 & 7.5 \\ \text { BP4 } & 6.2 & 6.0 & 5.6 & 5.7 \\ \text { BP5 } & 4.4 & 4.3 & 4.2 & 4.3 \\ \text { S } & & & & 0.7 \\ \text { BP1 } & 1.1 & 0.9 & 1.1 & 1.5 \\ \text { BP2 } & 2.3 & 2.1 & 2.4 & 2.3 \\ \text { BP3 } & 2.0 & 2.1 & 6.1 & 6.2 \\ \text { BP4 } & 6.6 & 6.4 & 5.4 & 5.5 \\ \text { BP5 } & 6.1 & 5.9 & & \end{array}$

Absolute energies (E), enthalpies (H), RRHO free energies (G), and quasi-RRHO free energies (Grimme G), in hartrees, along with the corresponding relative values in $\mathrm{kcal} / \mathrm{mol}$ and predicted ee's, for catalyst $2 \mathrm{a}$ ( $\mathrm{R}=\mathrm{Me})$ (only relatively low-lying TS structures considered)

$\begin{array}{lcccc}\text { Geometry } & \mathrm{E} & \mathrm{H} & \mathrm{G} & \text { G_Grimme } \\ \text { R } & 74.3 \% & 73.4 \% & 66.8 \% & 71.8 \% \\ \text { i.bp1 } & -2857.436919 & -2856.882692 & -2856.938093 & -2856.934568 \\ \text { i.bp2 } & -2857.442193 & -2856.888023 & -2856.942503 & -2856.939524 \\ \text { i.bp6 } & -2857.436899 & -2856.882710 & -2856.937918 & -2856.934519 \\ \text { i.bp7 } & -2857.442148 & -2856.887907 & -2856.942789 & -2856.939600\end{array}$




$\begin{array}{lllll}\text { ii.BP1 } & -2857.437210 & -2856.882923 & -2856.938238 & -2856.934766 \\ \text { ii.BP2 } & -2857.442616 & -2856.888413 & -2856.942839 & -2856.939890 \\ \text { iii.BP1 } & -2857.436598 & -2856.882464 & -2856.937860 & -2856.934356 \\ \text { iii.BP2 } & -2857.441590 & -2856.887288 & -2856.941948 & -2856.938717 \\ \text { S } & & & & \\ \text { i.bp1 } & -2857.436482 & -2856.882240 & -2856.937401 & -2856.934048 \\ \text { i.bp2 } & -2857.440039 & -2856.885654 & -2856.940360 & -2856.937157 \\ \text { i.bp3 } & -2857.439302 & -2856.884486 & -2856.938886 & -2856.935674 \\ \text { i.bp6 } & -2857.436372 & -2856.882839 & -2856.936669 & -2856.933675 \\ \text { i.bp7 } & -2857.441550 & -2856.887423 & -2856.942233 & -2856.939096 \\ \text { i.bp8 } & -2857.439602 & -2856.884675 & -2856.938655 & -2856.935785 \\ \text { ii.BP1 } & -2857.436711 & -2856.882380 & -2856.937369 & -2856.934063 \\ \text { ii.BP2 } & -2857.440453 & -2856.885823 & -2856.940036 & -2856.936909 \\ \text { ii.BP3 } & -2857.439767 & -2856.884986 & -2856.938827 & -2856.936018 \\ \text { iii.BP1 } & -2857.436122 & -2856.881973 & -2856.937034 & -2856.933739 \\ \text { iii.BP2 } & -2857.440965 & -2856.886926 & -2856.941609 & -2856.938338 \\ \text { iii.BP3 } & -2857.439126 & -2856.884262 & -2856.938176 & -2856.935258\end{array}$

Relative Energies

$\begin{array}{lcccc}\text { Geometry } & E & H(r e l) & G(r e l) & \text { G_Grimme }(r e 1) \\ \text { R } & 74.3 \% & 73.4 \% & 66.8 \% & 71.8 \% \\ \text { i.bp1 } & 3.6 & 3.6 & 3.0 & 3.3 \\ \text { i.bp2 } & 0.3 & 0.2 & 0.2 & 0.2 \\ \text { i.bp6 } & 3.6 & 3.6 & 3.1 & 3.4 \\ \text { i.bp7 } & 0.3 & 0.3 & 0.0 & 0.2 \\ \text { ii.BP1 } & 3.4 & 3.4 & 2.9 & 3.2 \\ \text { ii.BP2 } & 0.0 & 0.0 & 0.0 & 0.0 \\ \text { iii.BP1 } & 3.8 & 3.7 & 3.1 & 3.5 \\ \text { iii.BP2 } & 0.6 & 0.7 & 0.6 & 0.7 \\ \text { S } & & & & \\ \text { i.bp1 } & 3.8 & 3.9 & 3.4 & 3.7 \\ \text { i.bp2 } & 1.6 & 1.7 & 1.6 & 1.7 \\ \text { i.bp3 } & 2.1 & 2.5 & 2.5 & 2.6 \\ \text { i.bp6 } & 3.9 & 3.5 & 3.9 & 3.9 \\ \text { i.bp7 } & 0.7 & 0.6 & 0.4 & 0.5 \\ \text { i.bp8 } & 1.9 & 2.3 & 2.6 & 2.6 \\ \text { ii.BP1 } & 3.7 & 3.8 & 3.4 & 3.7 \\ \text { ii.BP2 } & 1.4 & 1.6 & 1.8 & 1.9 \\ \text { ii.BP3 } & 1.8 & 2.2 & 2.5 & 2.4 \\ \text { iii.BP1 } & 4.1 & 4.0 & 3.6 & 3.9 \\ \text { iii.BP2 } & 1.0 & 0.9 & 0.8 & 1.0 \\ \text { iii.BP3 } & 2.2 & 2.6 & 2.9 & 2.9 \\ & & & & \end{array}$


Absolute energies (E), enthalpies (H), RRHO free energies (G), and quasi-RRHO free energies (Grimme_G), in hartrees, along with the corresponding relative values in $\mathrm{kcal} / \mathrm{mol}$ and predicted ee's, for catalyst $2 \mathrm{a}$

\begin{tabular}{|c|c|c|c|c|}
\hline Geometry & $E$ & $\mathrm{H}$ & G & G_Grimme \\
\hline $\mathrm{R}$ & $82.8 \%$ & $83.8 \%$ & $63.3 \%$ & $74.6 \%$ \\
\hline i.bp1 & -2778.826923 & -2778.328204 & -2778.380660 & -2778.377339 \\
\hline i.bp2 & -2778.831595 & -2778.332913 & -2778.384226 & -2778.381443 \\
\hline i.bp3 & -2778.819061 & -2778.320356 & -2778.372689 & -2778.369425 \\
\hline i.bp 4 & -2778.820585 & -2778.321764 & -2778.373899 & -2778.370733 \\
\hline i.bp5 & -2778.824050 & -2778.325306 & -2778.377415 & -2778.374261 \\
\hline i.bp 6 & -2778.826868 & -2778.328223 & -2778.380840 & -2778.377452 \\
\hline i.bp 7 & -2778.831216 & -2778.332650 & -2778.384790 & -2778.381576 \\
\hline i.bp8 & -2778.819978 & -2778.321533 & -2778.374518 & -2778.370958 \\
\hline i.bpg & -2778.821182 & -2778.322616 & -2778.375260 & .371855 \\
\hline i.bp10 & -2778.824183 & -2778.325686 & -2778.378573 & -2778.375047 \\
\hline ii.BP1 & -2778.827310 & -2778.328666 & -2778.3 & -2778.37 \\
\hline ii.BP2 & -2778.832055 & -2778.333395 & -2778.384542 & -2778.381841 \\
\hline ii.BP3 & -2778.819 & -2778.32 & -2778.37 & -2778.3 \\
\hline ii.BP 4 & -2778.821197 & -2778.322350 & -2778.37 & -2778.371356 \\
\hline ii.BP 5 & -2778.824501 & -2778.325886 & -2778.3 & -2778.375017 \\
\hline iii.BP1 & -2778.826479 & -2778.327761 & -2778.380089 & -2778.376837 \\
\hline iii.BP2 & -2778.830574 & -2778.331959 & -2778.38 & -277 \\
\hline iii.BP3 & -2778.819449 & -2778.320955 & -2778.37 & -2778.370238 \\
\hline iii.BP 4 & -2778.820580 & -2778.321979 & -2778.374387 & -2778.371108 \\
\hline iii.BP5 & -2778.823728 & -2778.325052 & -2778.377822 & -2778.3 \\
\hline \multicolumn{5}{|l|}{$\mathrm{S}$} \\
\hline $\mathrm{p} 1$ & -2778.826441 & -2778.327813 & -2778.379999 & 3. 376812 \\
\hline i.bp2 & -2778.829455 & -2778.330710 & -2778.382882 & -2778.379664 \\
\hline i.bp3 & -2778.828852 & -2778.329672 & -2778.380437 & -2778.377853 \\
\hline op 4 & -2778.821537 & -2778.322690 & -2778.37 & -2778.371538 \\
\hline i.bp5 & -2778.823044 & -2778.324444 & -2778.376650 & -2778.373441 \\
\hline i.bp 6 & -2778.826328 & -2778.327648 & -2778.380145 & -2778.376804 \\
\hline . bp 7 & -2778.830389 & -2778.331770 & -2778.383791 & -2778.380624 \\
\hline i.bp8 & -2778.829171 & -2778.330137 & -2778.382240 & -2778.379025 \\
\hline i.bpg & -2778.821945 & -2778.323329 & -2778.375680 & -2778.372426 \\
\hline i.bp10 & -2778.823577 & -2778.324542 & -2778.376450 & -2778.373336 \\
\hline ii.BP1 & -2778.826798 & -2778.328150 & -2778.380448 & -2778.377210 \\
\hline ii.BP2 & -2778.829809 & -2778.331205 & -2778.383709 & -2778.380340 \\
\hline ii.BP3 & -2778.829313 & -2778.330392 & -2778.381895 & -2778.378972 \\
\hline i. BP 4 & -2778.822088 & -2778.323281 & -2778.375166 & -2778.372125 \\
\hline i. BP 5 & -2778.823544 & -2778.324874 & -2778.376917 & -2778.373782 \\
\hline ii.BP1 & -2778.825970 & -2778.327310 & -2778.379496 & 3.37630 \\
\hline
\end{tabular}




$\begin{array}{lllll}\text { iii.BP2 } & -2778.829931 & -2778.331269 & -2778.383347 & -2778.380157 \\ \text { iii.BP3 } & -2778.828662 & -2778.329714 & -2778.381132 & -2778.378275 \\ \text { iii.BP4 } & -2778.821407 & -2778.322711 & -2778.375034 & -2778.371796 \\ \text { iii.BP5 } & -2778.823082 & -2778.324093 & -2778.375999 & -2778.372901\end{array}$

Relative Energies

\begin{tabular}{|c|c|c|c|c|}
\hline Geometry & $\mathrm{E}$ & $\mathrm{H}(\mathrm{rel})$ & $G(r e l)$ & G_Grimme (rel) \\
\hline $\mathrm{R}$ & $82.8 \%$ & $83.8 \%$ & $63.3 \div$ & $74.6 \%$ \\
\hline i.bp1 & 3.2 & 3.3 & 2.6 & 2.8 \\
\hline i.bp2 & 0.3 & 0.3 & 0.4 & 0.2 \\
\hline i.bp3 & 8.2 & 8.2 & 7.6 & 7.8 \\
\hline i.bp4 & 7.2 & 7.3 & 6.8 & 7.0 \\
\hline i.bp5 & 5.0 & 5.1 & 4.6 & 4.8 \\
\hline i.bp 6 & 3.3 & 3.2 & 2.5 & 2.8 \\
\hline i.bp 7 & 0.5 & 0.5 & 0.0 & 0.2 \\
\hline i.bp8 & 7.6 & 7.4 & 6.4 & 6.8 \\
\hline i.bp 9 & 6.8 & 6.8 & 6.0 & 6.3 \\
\hline i.bp10 & 4.9 & 4.8 & 3.9 & $4 \cdot 3$ \\
\hline ii.BP1 & 3.0 & 3.0 & 2.1 & 2.4 \\
\hline ii.BP2 & 0.0 & 0.0 & 0.2 & 0.0 \\
\hline ii.BP3 & 7.8 & 7.9 & 7.7 & 7.7 \\
\hline ii.BP 4 & 6.8 & 6.9 & 6.4 & 6.6 \\
\hline ii.BP 5 & 4.7 & 4.7 & 4.1 & 4.3 \\
\hline iii.BP1 & 3.5 & 3.5 & 2.9 & 3.1 \\
\hline iii.BP2 & 0.9 & 0.9 & 0.4 & 0.6 \\
\hline iii.BP3 & 7.9 & 7.8 & 7.0 & 7.3 \\
\hline iii.BP4 & 7.2 & 7.2 & 6.5 & 6.7 \\
\hline iii.BP5 & 5.2 & 5.2 & 4.4 & 4.7 \\
\hline \multicolumn{5}{|l|}{ S } \\
\hline i.bp1 & 3.5 & 3.5 & 3.0 & 3.2 \\
\hline i.bp2 & 1.6 & 1.7 & 1.2 & 1.4 \\
\hline i.bp3 & 2.0 & 2.3 & 2.7 & 2.5 \\
\hline i.bp4 & 6.6 & 6.7 & 6.4 & 6.5 \\
\hline i.bp5 & 5.7 & 5.6 & 5.1 & 5.3 \\
\hline i.bp 6 & 3.6 & 3.6 & 2.9 & 3.2 \\
\hline i.bp 7 & 1.0 & 1.0 & 0.6 & 0.8 \\
\hline i.bp 8 & 1.8 & 2.0 & 1.6 & 1.8 \\
\hline i.bp 9 & 6.3 & 6.3 & 5.7 & 5.9 \\
\hline i.bp10 & 5.3 & 5.6 & 5.2 & 5.3 \\
\hline ii.BP1 & 3.3 & 3.3 & 2.7 & 2.9 \\
\hline ii.BP2 & 1.4 & 1.4 & 0.7 & 0.9 \\
\hline ii.BP3 & 1.7 & 1.9 & 1.8 & 1.8 \\
\hline ii.BP 4 & 6.3 & 6.3 & 6.0 & 6.1 \\
\hline
\end{tabular}




$\begin{array}{lllll}\text { ii.BP5 } & 5.3 & 5.3 & 4.9 & 5.1 \\ \text { iii.BP1 } & 3.8 & 3.8 & 3.3 & 3.5 \\ \text { iii.BP2 } & 1.3 & 1.3 & 0.9 & 1.1 \\ \text { iii.BP3 } & 2.1 & 2.3 & 2.3 & 2.2 \\ \text { iii.BP4 } & 6.7 & 6.7 & 6.1 & 6.3 \\ \text { iii.BP5 } & 5.6 & 5.8 & 5.5 & 5.6\end{array}$

Absolute energies (E), enthalpies (H), RRHO free energies (G), and quasi-RRHO free energies (Grimme G), in hartrees, along with the corresponding relative values in $\mathrm{kcal} / \mathrm{mol}$ and predicted ee's, for catalyst $2 \mathrm{~b}$

\begin{tabular}{|c|c|c|c|c|}
\hline Geometry & E & $\mathrm{H}$ & G & G_Grimme \\
\hline $\mathrm{R}$ & $93.8 \%$ & $94.2 \%$ & $88.9 \%$ & $92.2 \%$ \\
\hline i.bp1 & -2977.268136 & -2976.785127 & -2976.838713 & -2976.835560 \\
\hline i.bp2 & - & - & - & - \\
\hline i.bp3 & -2977.259297 & -2976.776304 & -2976.829557 & -2976.826604 \\
\hline i.bp4 & - & - & - & - \\
\hline i.bp5 & -2977.265018 & -2976.781935 & -2976.835773 & -2976.832468 \\
\hline i.bp 6 & -2977.268085 & -2976.785066 & -2976.838967 & -2976.835646 \\
\hline i.bp 7 & -2977.272339 & -2976.789403 & -2976.842904 & -2976.839727 \\
\hline i.bp8 & -2977.260364 & -2976.777166 & -2976.831312 & -2976.827850 \\
\hline i.bpg & -2977.262081 & -2976.779186 & -2976.832921 & -2976.8 \\
\hline i.bp10 & -2977.265401 & -2976.782472 & -2976.836310 & -2976.833029 \\
\hline ii.BP1 & -2977.268684 & -2976.785591 & -2976.839240 & -2976.836035 \\
\hline ii.BP2 & -2977.273678 & -2976.790841 & -2976.843900 & -2976.840994 \\
\hline ii.BP3 & -2977.260021 & -2976.776877 & -2976.829957 & -2976.827077 \\
\hline ii.BP 4 & -2977.262146 & -2976.779009 & -2976.832370 & -2976.829355 \\
\hline ii.BP5 & -2977.265722 & -2976.782664 & -2976.836360 & -2976.833136 \\
\hline iii.BP1 & -2977.267532 & -2976.784506 & -2976.838239 & -2976.835016 \\
\hline iii.BP2 & -2977.271755 & -2976.788823 & -2976.842115 & -2976.839074 \\
\hline iii.BP3 & -2977.259662 & -2976.776385 & -2976.829951 & -2976.826791 \\
\hline iii.BP 4 & - & - & - & - \\
\hline iii.BP 5 & -2977.26 & 976.781785 & 76.835573 & -2976.83 \\
\hline \\
\hline i.bp1 & -2977.267960 & -2976.785096 & -2976.838915 & -2976.835656 \\
\hline i.bp2 & -2977.270279 & -2976.787375 & -2976.840853 & -2976.837740 \\
\hline i.bp3 & -2977.269744 & -2976.786598 & -2976.839470 & -2976.836630 \\
\hline i.bp 4 & -2977.261480 & -2976.778573 & -2976.832773 & -2976.829324 \\
\hline i.bp5 & -2977.263181 & -2976.780083 & -2976.833538 & -2976.830457 \\
\hline i.bp 6 & -2977.267819 & -2976.784860 & -2976.838732 & -2976.835439 \\
\hline i.bp 7 & -2977.270719 & -2976.788087 & -2976.841794 & -2976.838556 \\
\hline i.bp8 & -2977.270330 & -2976.787138 & -2976.840070 & -2976.837196 \\
\hline
\end{tabular}




$\begin{array}{lllll}\text { i.bp9 } & -2977.262105 & -2976.779105 & -2976.832891 & -2976.829652 \\ \text { i.bp10 } & -2977.263175 & -2976.780305 & -2976.833915 & -2976.830800 \\ \text { ii.BP1 } & -2977.268450 & -2976.785512 & -2976.839313 & -2976.836051 \\ \text { ii.BP2 } & -2977.270695 & -2976.787807 & -2976.841494 & -2976.838258 \\ \text { ii.BP3 } & -2977.270337 & -2976.787386 & -2976.840703 & -2976.837614 \\ \text { ii.BP4 } & -2977.262231 & -2976.779177 & -2976.832633 & -2976.829536 \\ \text { ii.BP5 } & -2977.263792 & -2976.780623 & -2976.833849 & -2976.830877 \\ \text { iii.BP1 } & -2977.267322 & -2976.784435 & -2976.838237 & -2976.834988 \\ \text { iii.BP2 } & -2977.270324 & -2976.787205 & -2976.840180 & -2976.837289 \\ \text { iii.BP3 } & -2977.269678 & -2976.786507 & -2976.839716 & -2976.836700 \\ \text { iii.BP4 } & -2977.261387 & -2976.778489 & -2976.832459 & -2976.829141 \\ \text { iii.BP5 } & -2977.262560 & -2976.779778 & -2976.833778 & -2976.830469\end{array}$

Relative Energies

\begin{tabular}{|c|c|c|c|c|}
\hline Geometry & E & $\mathrm{H}(\mathrm{rel})$ & $\mathrm{G}(r e l)$ & G_Grimme (rel) \\
\hline $\mathrm{R}$ & $93.8 \%$ & $94.2 \%$ & $88.9 \%$ & $92.2 \%$ \\
\hline i.bp1 & 3.5 & 3.6 & 3.3 & 3.4 \\
\hline i.bp2 & - & - & - & - \\
\hline i.bp3 & 9.0 & 9.1 & 9.0 & 9.0 \\
\hline i.bp4 & - & - & - & - \\
\hline i.bp5 & 5.4 & 5.6 & 5.1 & 5.4 \\
\hline i.bp 6 & 3.5 & 3.6 & 3.1 & 3.4 \\
\hline i.bp 7 & 0.8 & 0.9 & 0.6 & 0.8 \\
\hline i.bp8 & 8.4 & 8.6 & 7.9 & 8.2 \\
\hline i.bpg & 7.3 & 7.3 & 6.9 & 7.1 \\
\hline i.bp10 & 5.2 & 5.3 & 4.8 & 5.0 \\
\hline ii.BP1 & 3.1 & 3.3 & 2.9 & 3.1 \\
\hline ii.BP2 & 0.0 & 0.0 & 0.0 & 0.0 \\
\hline ii.BP3 & 8.6 & 8.8 & 8.7 & 8.7 \\
\hline ii.BP 4 & 7.2 & 7.4 & 7.2 & 7.3 \\
\hline ii.BP 5 & 5.0 & 5.1 & 4.7 & 4.9 \\
\hline iii.BP1 & 3.9 & 4.0 & 3.6 & 3.8 \\
\hline iii.BP2 & 1.2 & 1.3 & 1.1 & 1.2 \\
\hline iii.BP3 & 8.8 & 9.1 & 8.8 & 8.9 \\
\hline iii.BP 4 & - & - & - & - \\
\hline iii.BP 5 & 5.6 & 5.7 & 5.2 & 5.4 \\
\hline \multicolumn{5}{|l|}{ S } \\
\hline i.bp1 & 3.6 & 3.6 & 3.1 & 3.3 \\
\hline i.bp2 & 2.1 & 2.2 & 1.9 & 2.0 \\
\hline i.bp3 & 2.5 & 2.7 & 2.8 & 2.7 \\
\hline i.bp4 & 7.7 & 7.7 & 7.0 & 7.3 \\
\hline i.bp5 & 6.6 & 6.8 & 6.5 & 6.6 \\
\hline i.bp6 & 3.7 & 3.8 & 3.2 & 3.5 \\
\hline
\end{tabular}




$\begin{array}{lllll}\text { i.bp7 } & 1.9 & 1.7 & 1.3 & 1.5 \\ \text { i.bp8 } & 2.1 & 2.3 & 2.4 & 2.4 \\ \text { i.bp9 } & 7.3 & 7.4 & 6.9 & 7.1 \\ \text { i.bp10 } & 6.6 & 6.6 & 6.3 & 6.4 \\ \text { ii.BP1 } & 3.3 & 3.3 & 2.9 & 3.1 \\ \text { ii.BP2 } & 1.9 & 1.9 & 1.5 & 1.7 \\ \text { ii.BP3 } & 2.1 & 2.2 & 2.0 & 2.1 \\ \text { ii.BP4 } & 7.2 & 7.3 & 7.1 & 7.2 \\ \text { ii.BP5 } & 6.2 & 6.4 & 6.3 & 6.3 \\ \text { iii.BP1 } & 4.0 & 4.0 & 3.6 & 3.8 \\ \text { iii.BP2 } & 2.1 & 2.3 & 2.3 & 2.3 \\ \text { iii.BP3 } & 2.5 & 2.7 & 2.6 & 2.7 \\ \text { iii.BP4 } & 7.7 & 7.8 & 7.2 & 7.4 \\ \text { iii.BP5 } & 7.0 & 6.9 & 6.4 & 6.6\end{array}$

Absolute energies (E), enthalpies (H), RRHO free energies (G), and quasi-RRHO free energies (Grimme_G), in hartrees, along with the corresponding relative values in $\mathrm{kcal} / \mathrm{mol}$ and predicted ee's, for catalyst $2 \mathrm{c}(\mathrm{R}=\mathrm{Me})$ (only relatively low-lying TS structures considered)

$\begin{array}{lcccc}\text { Geometry } & \text { E } & \text { H } & \text { G } & \text { G_Grimme } \\ \text { R } & 94.9 \% & 94.9 \% & 94.4 \% & 94.6 \% \\ \text { i.bp1 } & -3776.67301 & -3776.13777 & -3776.19659 & -3776.1928 \\ \text { i.bp2 } & -3776.67849 & -3776.14302 & -3776.20038 & -3776.19717 \\ \text { i.bp6 } & -3776.67299 & -3776.13755 & -3776.19634 & -3776.19244 \\ \text { i.bp7 } & -3776.67745 & -3776.14213 & -3776.20025 & -3776.1966 \\ \text { ii.BP1 } & -3776.67327 & -3776.13787 & -3776.19623 & -3776.19246 \\ \text { ii.BP2 } & -3776.67884 & -3776.14346 & -3776.20119 & -3776.1978 \\ \text { iii.BP1 } & -3776.67271 & -3776.13722 & -3776.19517 & -3776.19167 \\ \text { iii.BP2 } & -3776.6771 & -3776.14156 & -3776.19916 & -3776.19575 \\ \text { S } & & & & \\ \text { i.bp1 } & -3776.67279 & -3776.13754 & -3776.19591 & -3776.19228 \\ \text { i.bp2 } & -3776.67591 & -3776.14049 & -3776.19845 & -3776.19501 \\ \text { i.bp3 } & -3776.67406 & -3776.13823 & -3776.1952 & -3776.19212 \\ \text { i.bp6 } & -3776.67252 & -3776.13713 & -3776.19536 & -3776.1917 \\ \text { i.bp7 } & -3776.67613 & -3776.14077 & -3776.19863 & -3776.19514 \\ \text { i.bp8 } & -3776.67399 & -3776.1384 & -3776.19621 & -3776.19273 \\ \text { ii.BP1 } & -3776.67294 & -3776.13745 & -3776.19535 & -3776.19174 \\ \text { ii.BP2 } & -3776.67619 & -3776.14071 & -3776.19819 & -3776.19486 \\ \text { ii.BP3 } & -3776.67444 & -3776.13878 & -3776.1962 & -3776.1929 \\ \text { iii.BP1 } & -3776.67232 & -3776.13702 & -3776.19542 & -3776.1917 \\ \text { iii.BP2 } & -3776.67588 & -3776.14059 & -3776.19812 & -3776.19489 \\ \text { iii.BP3 } & -3776.67365 & -3776.13798 & -3776.1954 & -3776.19212\end{array}$


Relative Energies

\begin{tabular}{|c|c|c|c|c|}
\hline Geometry & $E$ & $\mathrm{H}(r e l)$ & $G(r e l)$ & G_Grimme(rel) \\
\hline $\mathrm{R}$ & $94.9 \%$ & $94.9 \%$ & $94.4 \%$ & $94.6 \%$ \\
\hline i.bp1 & 3.7 & 3.6 & 2.9 & 3.1 \\
\hline i.bp2 & 0.2 & 0.3 & 0.5 & 0.4 \\
\hline i.bp 6 & 3.7 & 3.7 & 3.0 & 3.4 \\
\hline i.bp 7 & 0.9 & 0.8 & 0.6 & 0.8 \\
\hline ii.BP1 & 3.5 & 3.5 & 3.1 & 3.4 \\
\hline ii.BP2 & 0.0 & 0.0 & 0.0 & 0.0 \\
\hline iii.BP1 & 3.8 & 3.9 & 3.8 & 3.8 \\
\hline iii.BP2 & 1.1 & 1.2 & 1.3 & 1.3 \\
\hline \multicolumn{5}{|l|}{ S } \\
\hline i.bp1 & 3.8 & 3.7 & 3.3 & 3.5 \\
\hline i.bp2 & 1.8 & 1.9 & 1.7 & 1.8 \\
\hline i.bp3 & 3.0 & 3.3 & 3.8 & 3.6 \\
\hline i.bp 6 & 4.0 & 4.0 & 3.7 & 3.8 \\
\hline i.bp 7 & 1.7 & 1.7 & 1.6 & 1.7 \\
\hline i.bp8 & 3.0 & 3.2 & 3.1 & 3.2 \\
\hline ii.BP1 & 3.7 & 3.8 & 3.7 & 3.8 \\
\hline ii.BP2 & 1.7 & 1.7 & 1.9 & 1.8 \\
\hline ii.BP3 & 2.8 & 2.9 & 3.1 & 3.1 \\
\hline iii.BP1 & 4.1 & 4.0 & 3.6 & 3.8 \\
\hline iii.BP2 & 1.9 & 1.8 & 1.9 & 1.8 \\
\hline iii.BP3 & 3.3 & 3.4 & 3.6 & 3.6 \\
\hline
\end{tabular}

Absolute energies (E), enthalpies (H), RRHO free energies (G), and quasi-RRHO free energies (Grimme_G), in hartrees, along with the corresponding relative values in $\mathrm{kcal} / \mathrm{mol}$ and predicted ee's, for catalyst 2c

$\begin{array}{lcccc}\text { Geometry } & \text { E } & \text { H } & \text { G } & \text { G_Grimme } \\ \text { R } & 95.8 \% & 96.7 \% & 94.8 \% & 95.9 \% \\ \text { i.bp1 } & -3698.061525 & -3697.581665 & -3697.636733 & -3697.633292 \\ \text { i.bp2 } & -3698.066800 & -3697.586732 & -3697.640696 & -3697.637739 \\ \text { i.bp3 } & -3698.052394 & -3697.572666 & -3697.627299 & -3697.624114 \\ \text { i.bp4 } & -3698.054510 & -3697.574635 & -3697.629750 & -3697.626313 \\ \text { i.bp5 } & -3698.058171 & -3697.578328 & -3697.633075 & -3697.629751 \\ \text { i.bp6 } & -3698.061547 & -3697.581881 & -3697.637181 & -3697.633638 \\ \text { i.bp7 } & -3698.065652 & -3697.585667 & -3697.640051 & -3697.636861 \\ \text { i.bp8 } & -3698.053123 & -3697.573427 & -3697.628698 & -3697.625181 \\ \text { i.bp9 } & -3698.054984 & -3697.575143 & -3697.630328 & -3697.626846 \\ \text { i.bp10 } & -3698.058275 & -3697.578592 & -3697.633376 & -3697.630071 \\ \text { i..BP1 } & -3698.062225 & -3697.582534 & -3697.637702 & -3697.634229 \\ \text { ii.BP2 } & -3698.067471 & -3697.587660 & -3697.642008 & -3697.638880 \\ \text { ii.BP3 } & -3698.053279 & -3697.573820 & -3697.628682 & -3697.625403\end{array}$




\begin{tabular}{|c|c|c|c|c|}
\hline 4 & 83 & -36 & -36 & -3 \\
\hline i. BP5 & 3698.059021 & -3697.579307 & -3697.634122 & -3697.630787 \\
\hline i.BP1 & 3698.060844 & -3697.580907 & -3697.635962 & 32506 \\
\hline i.BP2 & -3698.064876 & -3697.585036 & -3697.639673 & -3697.636375 \\
\hline . BP 3 & -3698.0 & 54 & 39 & -3 \\
\hline 1 & -3698.054108 & .574283 & .629380 & -36 \\
\hline 5 & -36 & -36 & 81 & -3 \\
\hline \\
\hline$\perp$ & 32 & -30 & -3 & -3 \\
\hline 2 & -3698.0 & 7.584363 & -3 & -3 \\
\hline 3 & -3698.062 & -3697.582335 & 7.636194 & -36 \\
\hline bp4 & -36 & -36 & -36 & -3 \\
\hline bp5 & -3698.051775 & -3697.572089 & -3697.627239 & -3697.623817 \\
\hline bp 6 & -3698.061036 & -3697.581499 & -3697.636900 & -3697 . \\
\hline bp 7 & -3698.064081 & -3697.584246 & -3697.639258 & 5785 \\
\hline 8 & -36 & -36 & 7.636928 & -3 \\
\hline op9 & -369 & -36 & -36 & -3 \\
\hline bp10 & -3698.051340 & 7.571728 & 7.626921 & -3697 \\
\hline BP1 & -3698.061853 & -3697.582410 & -3697.637864 & -3697.634243 \\
\hline BP2 & -3698.064970 & -3697.584816 & -3697.639077 & -3697.635990 \\
\hline $\mathrm{BP} 3$ & -36 & .583156 & -36 & -3 \\
\hline BP 4 & -369 & -36 & -36 & 634 \\
\hline i.BP 5 & -3698.052462 & -3697.572798 & -3697.627762 & -3697.624437 \\
\hline i.BP1 & -3698.060409 & -3697.580691 & -3697.636167 & -3697.632507 \\
\hline i.BP2 & -3698.063349 & -3697.583434 & -3697.638098 & -3697.634797 \\
\hline. $\mathrm{BP} 3$ & -3698.061922 & -3697.581726 & -3697.635675 & -3697.632724 \\
\hline BP 4 & -3698.054691 & -3697.574927 & -3697.630210 & -3697.626649 \\
\hline. BP 5 & -3698.050681 & -3697.571066 & -3697.626442 & -3697.62293 \\
\hline
\end{tabular}

Relative Energies

$\begin{array}{lcccc}\text { Geometry } & E & \text { H }(r e l) & \text { G(rel) } & \text { G_Grimme (rel) } \\ \text { R } & 95.8 \% & 96.7 \% & 94.8 \% & 95.9 \% \\ \text { i.bp1 } & 3.7 & 3.8 & 3.3 & 3.5 \\ \text { i.bp2 } & 0.4 & 0.6 & 0.8 & 0.7 \\ \text { i.bp3 } & 9.5 & 9.4 & 9.2 & 9.3 \\ \text { i.bp4 } & 8.1 & 8.2 & 7.7 & 7.9 \\ \text { i.bp5 } & 5.8 & 5.9 & 5.6 & 5.7 \\ \text { i.bp6 } & 3.7 & 3.6 & 3.0 & 3.3 \\ \text { i.bp7 } & 1.1 & 1.3 & 1.2 & 1.3 \\ \text { i.bp8 } & 9.0 & 8.9 & 8.4 & 8.6 \\ \text { i.bp9 } & 7.8 & 7.9 & 7.3 & 7.6 \\ \text { i.bp10 } & 5.8 & 5.7 & 5.4 & 5.5 \\ \text { i. } 1.3 P 1 & 3.3 & 3.2 & 2.7 & 2.9\end{array}$




\begin{tabular}{|c|c|c|c|c|}
\hline ii.BP2 & 0.0 & 0.0 & 0.0 & 0.0 \\
\hline ii.BP3 & 8.9 & 8.7 & 8.4 & 8.5 \\
\hline ii.BP 4 & 7.6 & 7.7 & 7.4 & 7.5 \\
\hline ii.BP5 & 5.3 & 5.2 & 4.9 & 5.1 \\
\hline iii.BP1 & 4.2 & 4.2 & 3.8 & 4.0 \\
\hline iii.BP2 & 1.6 & 1.6 & 1.5 & 1.6 \\
\hline iii.BP3 & 9.6 & 9.5 & 8.9 & 9.1 \\
\hline iii.BP 4 & 8.4 & 8.4 & 7.9 & 8.1 \\
\hline iii.BP5 & 6.3 & 6.3 & 6.1 & 6.2 \\
\hline \multicolumn{5}{|l|}{ S } \\
\hline i.bp1 & 3.9 & 3.8 & 3.2 & 3.5 \\
\hline i.bp2 & 2.1 & 2.1 & 1.9 & 2.0 \\
\hline i.bp3 & 3.2 & 3.3 & 3.6 & 3.5 \\
\hline i.bp4 & 7.8 & 7.8 & 7.2 & 7.4 \\
\hline i.bp5 & 9.8 & 9.8 & 9.3 & 9.5 \\
\hline i.bp 6 & 4.0 & 3.9 & 3.2 & 3.5 \\
\hline i.bp 7 & 2.1 & 2.1 & 1.7 & 1.9 \\
\hline i.bp8 & 3.0 & 3.1 & 3.2 & 3.2 \\
\hline i.bp9 & 7.5 & 7.5 & 7.1 & 7.3 \\
\hline i.bp10 & 10.1 & 10.0 & 9.5 & 9.6 \\
\hline ii.BP1 & 3.5 & 3.3 & 2.6 & 2.9 \\
\hline ii.BP2 & 1.6 & 1.8 & 1.8 & 1.8 \\
\hline ii.BP3 & 2.7 & 2.8 & 3.1 & 3.0 \\
\hline ii.BP 4 & 7.4 & 7.3 & 6.9 & 7.1 \\
\hline ii.BP5 & 9.4 & 9.3 & 8.9 & 9.1 \\
\hline iii.BP1 & 4.4 & 4.4 & 3.7 & 4.0 \\
\hline iii.BP2 & 2.6 & 2.7 & 2.5 & 2.6 \\
\hline iii.BP3 & 3.5 & 3.7 & 4.0 & 3.9 \\
\hline iii.BP 4 & 8.0 & 8.0 & 7.4 & 7.7 \\
\hline iii.BP5 & 10.5 & 10.4 & 9.8 & 10.0 \\
\hline
\end{tabular}

Absolute energies (E), enthalpies (H), RRHO free energies (G), and quasi-RRHO free energies (Grimme G), in hartrees, along with the corresponding relative values in $\mathrm{kcal} / \mathrm{mol}$ and predicted ee's, for catalyst $2 \mathrm{~d}$

$\begin{array}{lcccc}\text { Geometry } & \text { E } & \text { H } & \text { G } & \text { G_Grimme } \\ \text { R } & 77.3 \% & 87.5 \% & 94.1 \% & 93.1 \% \\ \text { i.bp8 } & -2857.431876 & -2856.877752 & -2856.932545 & -2856.929165 \\ \text { i.bp9 } & -2857.433669 & -2856.879455 & -2856.93386 & -2856.930645 \\ \text { i.bp10 } & -2857.437395 & -2856.883164 & -2856.937495 & -2856.934331 \\ \text { i..BP1 } & -2857.441235 & -2856.887339 & -2856.942317 & -2856.938895 \\ \text { i..BP2 } & -2857.445834 & -2856.891936 & -2856.946241 & -2856.943185 \\ \text { S } & & & & \end{array}$




$\begin{array}{lllll}\text { i.bp8 } & -2857.442094 & -2856.887428 & -2856.940546 & -2856.93793 \\ \text { i.bp9 } & -2857.435562 & -2856.881839 & -2856.937259 & -2856.933745 \\ \text { ii.BP1 } & -2857.440593 & -2856.886675 & -2856.941412 & -2856.938159 \\ \text { ii.BP2 } & -2857.444535 & -2856.890244 & -2856.94407 & -2856.941107 \\ \text { ii.BP3 } & -2857.442361 & -2856.887892 & -2856.941249 & -2856.938509 \\ \text { ii.BP5 } & -2857.430861 & -2856.876685 & -2856.930832 & -2856.927739\end{array}$

Relative Energies

$\begin{array}{lcccc}\text { Geometry } & E & \text { H }(r e l) & \text { G }(r e l) & \text { G_Grimme }(r e l) \\ \text { R } & 77.3 \% & 87.5 \% & 94.1 \% & 93.1 \% \\ \text { i.bp8 } & 8.8 & 8.9 & 8.6 & 8.8 \\ \text { i.bp9 } & 7.6 & 7.8 & 7.8 & 7.9 \\ \text { i.bp10 } & 5.3 & 5.5 & 5.5 & 5.6 \\ \text { ii.BP1 } & 2.9 & 2.9 & 2.5 & 2.7 \\ \text { ii.BP2 } & 0.0 & 0.0 & 0.0 & 0.0 \\ \text { S } & & & & \\ \text { i.bp8 } & 2.3 & 2.8 & 3.6 & 3.3 \\ \text { i.bp9 } & 6.4 & 6.3 & 5.6 & 5.9 \\ \text { ii.BP1 } & 3.3 & 3.3 & 3.0 & 3.2 \\ \text { ii.BP2 } & 0.8 & 1.1 & 1.4 & 1.3 \\ \text { ii.BP3 } & 2.2 & 2.5 & 3.1 & 2.9 \\ \text { ii.BP5 } & 9.4 & 9.6 & 9.7 & 9.7\end{array}$

Absolute energies (E), enthalpies (H), RRHO free energies (G), and quasi-RRHO free energies (Grimme_G), in hartrees, along with the corresponding relative values in $\mathrm{kcal} / \mathrm{mol}$ and predicted ee's, for catalyst $2 e(\mathrm{R}=\mathrm{Me})$ (only relatively low-lying TS structures considered)

$\begin{array}{lcccc}\text { Geometry } & \text { E } & \text { H } & \text { G } & \text { G_Grimme } \\ \text { R } & 98.6 \% & 97.1 \% & 88.4 \% & 92.9 \% \\ \text { ii.BP1 } & -3531.4164 & -3530.85232 & -3530.91533 & -3530.91103 \\ \text { ii.BP2 } & -3531.42236 & -3530.85798 & -3530.91973 & -3530.91603 \\ \text { S } & & & & \\ \text { ii.BP1 } & -3531.41654 & -3530.85225 & -3530.91435 & -3530.91048 \\ \text { ii.BP2 } & -3531.41928 & -3530.85537 & -3530.918 & -3530.91399 \\ \text { ii.BP3 } & -3531.41536 & -3530.8508 & -3530.91272 & -3530.9089\end{array}$

Relative Energies

$\begin{array}{lcccc}\text { Geometry } & E & \text { H }(r e l) & \text { G }(r e l) & \text { G_Grimme }(r e l) \\ \text { R } & 98.6 \% & 97.1 \% & 88.4 \% & 92.9 \% \\ \text { ii.BP1 } & 3.7 & 3.6 & 2.8 & 3.1 \\ \text { ii.BP2 } & 0.0 & 0.0 & 0.0 & 0.0 \\ \text { S } & & & & 3.5 \\ \text { ii.BP1 } & 3.7 & 3.6 & 3.4 & \end{array}$




$\begin{array}{rrrrr}\text { ii.BP2 } & 1.9 & 1.6 & 1.1 & 1.3 \\ \text { ii.BP3 } & 4.4 & 4.5 & 4.4 & 4.5\end{array}$

Absolute energies (E), enthalpies (H), RRHO free energies (G), and quasi-RRHO free energies (Grimme_G), in hartrees, along with the corresponding relative values in $\mathrm{kcal} / \mathrm{mol}$ and predicted ee's, for catalyst $2 e$

$\begin{array}{lcccc}\text { Geometry } & \mathrm{E} & \mathrm{H} & \mathrm{G} & \begin{array}{c}\text { G_Grimme } \\ \text { R }\end{array} \\ \text { ii.BP1 } & -3452.8 \% & 98.6 \% & 97.1 \% & 97.8 \% \\ \text { ii.BP2 } & -3452.808514 & -3452.299707 & -3452.358239 & -3452.354822 \\ \text { ii.BP3 } & -3452.792848 & -3452.2845 & -3452.344649 & -3452.340468 \\ \text { ii.BP4 } & -3452.795202 & -3452.286837 & -3452.34672 & -3452.342779 \\ \text { S } & & -3452.294243 & -3452.354114 & -3452.350061 \\ \text { ii.BP1 } & -3452.80282 & -3452.294386 & -3452.353926 & -3452.350032 \\ \text { ii.BP2 } & -3452.80533 & -3452.296651 & -3452.355594 & -3452.352022 \\ \text { ii.BP3 } & -3452.801508 & -3452.292529 & -3452.351254 & -3452.347697 \\ \text { ii.BP4 } & -3452.796135 & -3452.287285 & -3452.346862 & -3452.342967 \\ \text { ii.BP5 } & -3452.791154 & -3452.282698 & -3452.342569 & -3452.33861\end{array}$

\section{Relative Energies}

$\begin{array}{lcccc}\text { Geometry } & \mathrm{E} & \mathrm{H}(\mathrm{rel}) & \mathrm{G}(\mathrm{rel}) & \text { G_Grimme }(r e l) \\ \mathrm{R} & 98.8 \% & 98.6 \% & 97.1 \% & 97.8 \% \\ \text { ii.BP1 } & 3.6 & 3.4 & 2.6 & 3.0 \\ \text { ii.BP2 } & 0.0 & 0.0 & 0.0 & 0.0 \\ \text { ii.BP3 } & 9.8 & 9.5 & 8.5 & 9.0 \\ \text { ii.BP4 } & 8.4 & 8.1 & 7.2 & 7.6 \\ \mathrm{~S} & & & & \\ \text { ii.BP1 } & 3.6 & 3.3 & 2.7 & 3.0 \\ \text { ii.BP2 } & 2.0 & 1.9 & 1.7 & 1.8 \\ \text { ii.BP3 } & 4.4 & 4.5 & 4.4 & 4.5 \\ \text { ii.BP4 } & 7.8 & 7.8 & 7.1 & 7.4 \\ \text { ii.BP5 } & 10.9 & 10.7 & 9.8 & 10.2\end{array}$

Absolute energies (E), enthalpies (H), RRHO free energies (G), and quasi-RRHO free energies (Grimme_G), in hartrees, along with the corresponding relative values in $\mathrm{kcal} / \mathrm{mol}$ and predicted ee's, for catalyst $2 \mathrm{f}$

$\begin{array}{lcccc}\text { Geometry } & \mathrm{E} & \mathrm{H} & \mathrm{G} & \mathrm{G} \text { _Grimme } \\ & -9.9 \% & -36.4 \% & -6.3 \% & -33.4 \% \\ \text { R } & & & & \\ \text { i.bp8 } & -3014.616819 & -3013.949425 & -3014.009223 & -3014.005542 \\ \text { i. BP1 } & -3014.622265 & -3013.954616 & -3014.014546 & -3014.010784 \\ \text { i. B. BP2 } & -3014.624742 & -3013.957137 & -3014.016462 & -3014.012935 \\ \text { S } & & & & \end{array}$




\begin{tabular}{|c|c|c|c|c|}
\hline i.bp1 & -3014.619951 & -3013.952307 & -3014.012248 & -3014.008384 \\
\hline i.bp 7 & -3014.624875 & -3013.957618 & -3014.016566 & -3014.013383 \\
\hline i.bp8 & -3014.617203 & -3013.949694 & -3014.009338 & -3014.005711 \\
\hline i.bp10 & -3014.613732 & -3013.946701 & -3014.006461 & -3014.002798 \\
\hline ii.BP1 & -3014.620516 & & & \\
\hline ii.BP3 & -3014.61851 & & & \\
\hline ii.BP 4 & -3014.617155 & & & \\
\hline iii.BP3 & -3014.617203 & & & \\
\hline \multicolumn{5}{|c|}{ Relative Energies } \\
\hline Geometry & $\begin{array}{c}E \\
-9.9 \%\end{array}$ & $\begin{array}{l}H(r e l) \\
-36.4 \%\end{array}$ & $\begin{array}{c}G(r e l) \\
-6.3 \%\end{array}$ & $\begin{array}{c}\text { G_Grimme }(r e l) \\
-33.4 \%\end{array}$ \\
\hline \multicolumn{5}{|l|}{ R } \\
\hline i.bp8 & 5.1 & 5.1 & 4.6 & 6.9 \\
\hline ii.BP1 & 1.6 & 1.9 & 1.3 & 3.6 \\
\hline ii.BP2 & 0.1 & 0.3 & 0.1 & 2.3 \\
\hline \multicolumn{5}{|l|}{ S } \\
\hline i.bp1 & 3.1 & 3.3 & 2.7 & 5.1 \\
\hline i.bp 7 & 0.0 & 0.0 & 0.0 & 2.0 \\
\hline i.bp8 & 4.8 & 5.0 & 4.5 & 6.8 \\
\hline i.bp10 & 7.0 & 6.9 & 6.3 & 8.6 \\
\hline ii.BP1 & 2.7 & & & \\
\hline ii.BP3 & 4.0 & & & \\
\hline ii.BP 4 & 4.8 & & & \\
\hline iii.BP3 & 4.8 & & & \\
\hline \multicolumn{3}{|c|}{$\begin{array}{l}\text { Absolute energies (E), enthalpies (H), } \\
\text { free energies (Grimme G), in hartrees, } \\
\text { values in kcal/mol and predicted ee's, }\end{array}$} & \multicolumn{2}{|c|}{$\begin{array}{l}\text { RRHO free energies (G), and quasi-RRHO } \\
\text { along with the corresponding relative } \\
\text { for catalyst } 2 \mathrm{~g}\end{array}$} \\
\hline Geometry & $\mathrm{E}$ & $\mathrm{H}$ & G & G_Grimme \\
\hline $\mathrm{R}$ & $-18.1 \%$ & $-30.2 \%$ & $65.0 \%$ & $15.9 \%$ \\
\hline ii.BP1 & -3093.198919 & -3092.476128 & -3092.536898 & -3092.533436 \\
\hline ii.BP2 & -3093.201583 & -3092.478611 & -3092.539539 & -3092.535924 \\
\hline ii.BP3 & -3093.187312 & -3092.464442 & -3092.524333 & -3092.521212 \\
\hline ii.BP4 & -3093.190558 & -3092.467837 & -3092.529027 & -3092.525224 \\
\hline ii.BP5 & -3093.194404 & -3092.471626 & -3092.531707 & -3092.528459 \\
\hline \multicolumn{5}{|l|}{$\mathrm{S}$} \\
\hline ii.BP1 & -3093.197364 & -3092.474271 & -3092.535136 & -3092.531462 \\
\hline ii.BP2 & -3093.201817 & -3092.479007 & -3092.538587 & -3092.535736 \\
\hline ii.BP3 & -3093.194189 & -3092.471545 & -3092.53209 & -3092.52875 \\
\hline ii.BP 4 & -3093.192907 & -3092.470187 & -3092.530767 & -3092.527369 \\
\hline ii.BP 5 & -3093.182377 & -3092.459428 & -3092.519878 & -3092.516436 \\
\hline
\end{tabular}




\section{Relative Energies}

$\begin{array}{lcccc}\text { Geometry } & E & \text { H }(r e l) & \text { G }(r e l) & \text { G_Grimme }(r e l) \\ \text { R } & -18.1 \% & -30.2 \% & 65.0 \% & 15.9 \% \\ \text { ii.BP1 } & 1.8 & 1.8 & 1.7 & 1.6 \\ \text { ii.BP2 } & 0.1 & 0.2 & 0.0 & 0.0 \\ \text { ii.BP3 } & 9.1 & 9.1 & 9.5 & 9.2 \\ \text { ii.BP4 } & 7.1 & 7.0 & 6.6 & 6.7 \\ \text { ii.BP5 } & 4.7 & 4.6 & 4.9 & 4.7 \\ \text { S } & & & & \\ \text { ii.BP1 } & 2.8 & 3.0 & 2.8 & 2.8 \\ \text { ii.BP2 } & 0.0 & 0.0 & 0.6 & 0.1 \\ \text { ii.BP3 } & 4.8 & 4.7 & 4.7 & 4.5 \\ \text { ii.BP4 } & 5.6 & 5.5 & 5.5 & 5.4 \\ \text { ii.BP5 } & 12.2 & 12.3 & 12.3 & 12.2\end{array}$

Absolute energies (E), enthalpies (H), RRHO free energies (G), and quasi-RRHO free energies (Grimme_G), in hartrees, along with the corresponding relative values in $\mathrm{kcal} / \mathrm{mol}$ and predicted ee's, for catalyst $2 \mathrm{~h}$ ( $\mathrm{R}=\mathrm{Me})$ (only relatively low-lying TS structures considered)

\begin{tabular}{|c|c|c|c|c|}
\hline Geometry & $\mathrm{E}$ & $\mathrm{H}$ & G & G_Grimme \\
\hline $\mathrm{R}$ & $97.3 \%$ & $96.3 \%$ & $89.5 \%$ & $93.3 \%$ \\
\hline i.bp1 & -3009.64986 & -3009.07786 & -3009.13774 & -3009.13395 \\
\hline i.bp2 & - & - & - & - \\
\hline i.bp 6 & -3009.64988 & -3009.07766 & -3009.13697 & -3009.13338 \\
\hline i.bp 7 & -3009.65374 & -3009.08153 & -3009.14043 & -3009.13696 \\
\hline ii.BP1 & -3009.65025 & -3009.078 & -3009.13706 & -3009.13352 \\
\hline ii.BP2 & -3009.65522 & -3009.08304 & -3009.14173 & -3009.13847 \\
\hline iii.BP1 & -3009.64947 & -3009.07734 & -3009.13693 & -3009.13322 \\
\hline iii.BP2 & - & - & - & - \\
\hline \multicolumn{5}{|l|}{$S$} \\
\hline i.bp1 & -3009.65016 & -3009.07835 & -3009.13829 & -3009.13442 \\
\hline i.bp2 & - & - & - & - \\
\hline i.bp3 & -3009.65015 & -3009.07797 & -3009.13686 & -3009.13348 \\
\hline i.bp 6 & -3009.64992 & -3009.07771 & -3009.13675 & -3009.13323 \\
\hline i.bp 7 & -3009.65206 & -3009.08002 & -3009.13896 & -3009.13551 \\
\hline i.bp8 & -3009.65022 & -3009.07779 & -3009.13639 & -3009.13316 \\
\hline ii.BP1 & -3009.65037 & -3009.07819 & -3009.13704 & -3009.13356 \\
\hline ii.BP2 & -3009.65213 & -3009.08023 & -3009.13981 & -3009.13621 \\
\hline ii.BP3 & -3009.65071 & -3009.07855 & -3009.13722 & -3009.13395 \\
\hline iii.BP1 & -3009.6497 & -3009.07757 & -3009.13693 & -3009.13327 \\
\hline iii.BP2 & - & - & - & - \\
\hline iii.BP3 & -3009.64971 & -3009.07743 & -3009.13648 & -3009.13305 \\
\hline
\end{tabular}


Relative Energies

\begin{tabular}{|c|c|c|c|c|}
\hline Geometry & $\mathrm{E}$ & $\mathrm{H}(r e l)$ & G(rel) & G_Grimme(rel) \\
\hline $\mathrm{R}$ & $97.3 \%$ & $96.3 \%$ & $89.5 \%$ & $93.3 \%$ \\
\hline i.bp1 & 3.4 & 3.3 & 2.5 & 2.8 \\
\hline i.bp2 & - & - & - & - \\
\hline i.bp 6 & 3.3 & 3.4 & 3.0 & 3.2 \\
\hline i.bp 7 & 0.9 & 0.9 & 0.8 & 0.9 \\
\hline ii.BP1 & 3.1 & 3.2 & 2.9 & 3.1 \\
\hline ii.BP2 & 0.0 & 0.0 & 0.0 & 0.0 \\
\hline iii.BP1 & 3.6 & 3.6 & 3.0 & 3.3 \\
\hline iii.BP2 & - & - & - & - \\
\hline \multicolumn{5}{|l|}{ S } \\
\hline i.bp1 & 3.2 & 2.9 & 2.2 & 2.5 \\
\hline i.bp2 & - & - & - & - \\
\hline i.bp3 & 3.2 & 3.2 & 3.1 & 3.1 \\
\hline i.bp 6 & 3.3 & 3.3 & 3.1 & 3.3 \\
\hline i.bp 7 & 2.0 & 1.9 & 1.7 & 1.9 \\
\hline i.bp8 & 3.1 & 3.3 & 3.3 & 3.3 \\
\hline ii.BP1 & 3.0 & 3.0 & 2.9 & 3.1 \\
\hline ii.BP2 & 1.9 & 1.8 & 1.2 & 1.4 \\
\hline ii.BP3 & 2.8 & 2.8 & 2.8 & 2.8 \\
\hline iii.BP1 & 3.5 & 3.4 & 3.0 & 3.3 \\
\hline iii.BP2 & - & - & - & - \\
\hline iii.BP3 & 3.5 & 3.5 & 3.3 & 3.4 \\
\hline
\end{tabular}

Absolute energies (E), enthalpies (H), RRHO free energies (G), and quasi-RRHO free energies (Grimme_G), in hartrees, along with the corresponding relative values in $\mathrm{kcal} / \mathrm{mol}$ and predicted ee's, for catalyst $2 \mathrm{~h}$

\begin{tabular}{|c|c|c|c|c|}
\hline Geometry & $E$ & $\mathrm{H}$ & G & G_Grimme \\
\hline $\mathrm{R}$ & $97.5 \%$ & $98.0 \%$ & $96.7 \%$ & $97.5 \%$ \\
\hline i.bp1 & -2931.038533 & -2930.521937 & -2930.578057 & -2930.574681 \\
\hline i.bp 6 & -2931.038586 & -2930.522307 & -2930.579384 & -2930.575571 \\
\hline i.bp 7 & -2931.042007 & -2930.525536 & -2930.581652 & -2930.578267 \\
\hline i.bpg & -2931.033295 & -2930.516615 & -2930.572636 & -2930.569333 \\
\hline i. BP 1 & -2931.039387 & -2930.522947 & -2930.579303 & -2930.575836 \\
\hline ii.BP2 & -2931.043997 & -2930.527535 & -2930.583172 & -2930.580055 \\
\hline iii.BP1 & -2931.037797 & -2930.521217 & -2930.577501 & -2930.574056 \\
\hline iii.BP3 & -2931.029062 & -2930.512551 & -2930.569291 & -2930.565672 \\
\hline iii.BP5 & -2931.034062 & -2930.517555 & -2930.573337 & -2930.57009 \\
\hline \multicolumn{5}{|l|}{$\mathrm{C}$} \\
\hline i.bp1 & -2931.03888 & -2930.522448 & -2930.578709 & -2930.575253 \\
\hline i.bp3 & -2931.038649 & -2930.521936 & -2930.577246 & -2930.574262 \\
\hline bp5 & -2931.029379 & -2930.513036 & -2930.56961 & 6047 \\
\hline
\end{tabular}




$\begin{array}{lllll}\text { i.bp6 } & -2931.038601 & -2930.522347 & -2930.578992 & -2930.575376 \\ \text { i.bp7 } & -2931.039945 & -2930.523486 & -2930.579826 & -2930.57634 \\ \text { i.bp8 } & -2931.038975 & -2930.522068 & -2930.577282 & -2930.57436 \\ \text { ii.BP1 } & -2931.039506 & -2930.523063 & -2930.579417 & -2930.575922 \\ \text { ii.BP2 } & -2931.041104 & -2930.524495 & -2930.580274 & -2930.577092 \\ \text { ii.BP3 } & -2931.03962 & -2930.522804 & -2930.577996 & -2930.575075 \\ \text { iii.BP1 } & -2931.038025 & -2930.521588 & -2930.577858 & -2930.574397 \\ \text { iii.BP3 } & -2931.038058 & -2930.521468 & -2930.577038 & -2930.573988 \\ \text { iii.BP4 } & -2931.03089 & -2930.514501 & -2930.570961 & -2930.567474\end{array}$

Relative Energies

$\begin{array}{lcccc}\text { Geometry } & \text { E } & \text { H(rel) } & \text { G(rel) } & \text { G_Grimme (rel) } \\ \text { R } & 97.5 \% & 98.0 \% & 96.7 \% & 97.5 \% \\ \text { i.bp1 } & 3.4 & 3.5 & 3.2 & 3.4 \\ \text { i.bp6 } & 3.4 & 3.3 & 2.4 & 2.8 \\ \text { i.bp7 } & 1.2 & 1.3 & 1.0 & 1.1 \\ \text { i.bp9 } & 6.7 & 6.9 & 6.6 & 6.7 \\ \text { ii.BP1 } & 2.9 & 2.9 & 2.4 & 2.6 \\ \text { ii.BP2 } & 0.0 & 0.0 & 0.0 & 0.0 \\ \text { iii.BP1 } & 3.9 & 4.0 & 3.6 & 3.8 \\ \text { iii.BP3 } & 9.4 & 9.4 & 8.7 & 9.0 \\ \text { iii.BP5 } & 6.2 & 6.3 & 6.2 & 6.3 \\ \text { S } & & & & 3.0 \\ \text { i.bp1 } & 3.2 & 3.2 & 2.8 & 3.6 \\ \text { i.bp3 } & 3.4 & 3.5 & 3.7 & 8.8 \\ \text { i.bp5 } & 9.2 & 9.1 & 8.5 & 2.9 \\ \text { i.bp6 } & 3.4 & 3.3 & 2.6 & 2.3 \\ \text { i.bp7 } & 2.5 & 2.5 & 2.1 & 3.6 \\ \text { i.bp8 } & 3.2 & 3.4 & 3.7 & 2.6 \\ \text { ii.BP1 } & 2.8 & 2.8 & 2.4 & 1.9 \\ \text { ii.BP2 } & 1.8 & 1.9 & 1.8 & 3.1 \\ \text { ii.BP3 } & 2.7 & 3.0 & 3.2 & 3.6 \\ \text { iii.BP1 } & 3.7 & 3.7 & 3.3 & 3.8 \\ \text { iii.BP3 } & 3.7 & 3.8 & 3.8 & 7.9 \\ \text { iii.BP4 } & 8.2 & 8.2 & & \end{array}$

Absolute energies (E), enthalpies (H), RRHO free energies (G), and quasi-RRHO free energies (Grimme_G), in hartrees, along with the corresponding relative values in $\mathrm{kcal} / \mathrm{mol}$ and predicted ee's, for catalyst $2 i \quad(R=M e)$ (only relatively low-lying TS structures considered)

$\begin{array}{lcccc}\text { Geometry } & \text { E } & \text { H } & \text { G } & \text { G_Grimme } \\ \text { R } & 98.7 \% & 98.3 \% & 98.4 \% & 98.6 \% \\ \text { i.bp1 } & -3041.82422 & -3041.27272 & -3041.33199 & -3041.32836\end{array}$




$\begin{array}{lllll}\text { i.bp2 } & -3041.82987 & -3041.27813 & -3041.33657 & -3041.33332 \\ \text { i.bp6 } & -3041.82428 & -3041.27276 & -3041.33199 & -3041.3283 \\ \text { i.bp7 } & -3041.82837 & -3041.27666 & -3041.33512 & -3041.3317 \\ \text { S } & & & & \\ \text { i.bp1 } & -3041.82466 & -3041.27318 & -3041.33226 & -3041.32864 \\ \text { i.bp2 } & -3041.8262 & -3041.27463 & -3041.33324 & -3041.32981 \\ \text { i.bp3 } & -3041.82421 & -3041.27244 & -3041.33096 & -3041.32759 \\ \text { i.bp6 } & -3041.82441 & -3041.27273 & -3041.33146 & -3041.32798 \\ \text { i.bp7 } & -3041.82456 & -3041.27264 & -3041.33076 & -3041.32759 \\ \text { i.bp8 } & -3041.82646 & -3041.27485 & -3041.33303 & -3041.32978\end{array}$

Relative Energies

$\begin{array}{lcccc}\text { Geometry } & E & \mathrm{H}(\mathrm{rel}) & \mathrm{G}(\mathrm{rel}) & \text { G_Grimme (rel) } \\ \mathrm{R} & 98.7 \% & 98.3 \% & 98.4 \% & 98.6 \% \\ \text { i.bp1 } & 3.5 & 3.4 & 2.9 & 3.1 \\ \text { i.bp2 } & 0.0 & 0.0 & 0.0 & 0.0 \\ \text { i.bp6 } & 3.5 & 3.4 & 2.9 & 3.1 \\ \text { i.bp7 } & 0.9 & 0.9 & 0.9 & 1.0 \\ \mathrm{~S} & & & & 2.9 \\ \text { i.bp1 } & 3.3 & 3.1 & 2.7 & 2.2 \\ \text { i.bp2 } & 2.3 & 2.2 & 2.1 & 3.6 \\ \text { i.bp3 } & 3.6 & 3.6 & 3.5 & 3.3 \\ \text { i.bp6 } & 3.4 & 3.4 & 3.2 & 3.6 \\ \text { i.bp7 } & 3.3 & 3.4 & 3.6 & 2.2 \\ \text { i.bp8 } & 2.1 & 2.1 & 2.2 & \end{array}$

Absolute energies (E), enthalpies (H), RRHO free energies (G), and quasi-RRHO free energies (Grimme_G), in hartrees, along with the corresponding relative values in $\mathrm{kcal} / \mathrm{mol}$ and predicted ee's, for catalyst $2 i$

$\begin{array}{lcccc}\text { Geometry } & \mathrm{E} & \mathrm{H} & \mathrm{G} & \mathrm{G} \text { Grimme } \\ \text { R } & 99.5 \% & 99.4 \% & 98.1 \% & 98.9 \% \\ \text { i.bp1 } & -2963.211328 & -2962.715346 & -2962.771374 & -2962.767935 \\ \text { i.bp2 } & -2963.216499 & -2962.720413 & -2962.775316 & -2962.772415 \\ \text { i.bp6 } & -2963.211516 & -2962.715553 & -2962.771787 & -2962.768231 \\ \text { i.bp7 } & -2963.215132 & -2962.719002 & -2962.775034 & -2962.771504 \\ \text { ii.BP1 } & -2963.212377 & -2962.716242 & -2962.771942 & -2962.768645 \\ \text { ii.BP2 } & -2963.217209 & -2962.721084 & -2962.776084 & -2962.773121 \\ \text { iii.BP1 } & -2963.210565 & -2962.714579 & -2962.770595 & -2962.767185 \\ \text { iii.BP2 } & -2963.21444 & -2962.718181 & -2962.773364 & -2962.770267 \\ \text { iii.BP3 } & -2963.201075 & -2962.70516 & -2962.761593 & -2962.757933 \\ \text { iii.BP4 } & -2963.202322 & -2962.706466 & -2962.762845 & -2962.759313 \\ \text { iii.BP5 } & -2963.206834 & -2962.711001 & -2962.76689 & -2962.763539 \\ \text { S } & & & & \end{array}$




\begin{tabular}{|c|c|c|c|c|}
\hline i.bp1 & -2963.211868 & -2962.715923 & -2962.771669 & -2962.768344 \\
\hline i.bp2 & -2963.213173 & -2962.717226 & -2962.772775 & -2962.769583 \\
\hline i.bp3 & -2963.211088 & -2962.714739 & -2962.769515 & -2962.766578 \\
\hline i.bp6 & -2963.211638 & -2962.715715 & -2962.771659 & -2962.76823 \\
\hline i.bp 7 & -2963.212751 & -2962.716806 & -2962.772805 & -2962.769356 \\
\hline i.bp8 & -2963.211856 & -2962.715518 & -2962.770479 & -2962.767485 \\
\hline ii.BP1 & -2963.212602 & -2962.716479 & -2962.772102 & -2962.76882 \\
\hline ii.BP2 & -2963.213938 & -2962.717872 & -2962.77312 & -2962.770059 \\
\hline iii.BP1 & -2963.210977 & -2962.715029 & -2962.770701 & -2962.767432 \\
\hline iii.BP2 & -2963.212051 & -2962.715965 & -2962.771673 & -2962.768373 \\
\hline iii.BP3 & -2963.210691 & -2962.714597 & -2962.769594 & -2962.766596 \\
\hline iii.BP 4 & -2963.202639 & -2962.706823 & -2962.763041 & -2962.75957 \\
\hline iii.BP5 & -2963.2018 & -2962.705841 & -2962.76222 & -2962.758654 \\
\hline \multicolumn{5}{|c|}{ Relative Energies } \\
\hline Geometry & $\mathrm{E}$ & $\mathrm{H}(r e l)$ & $G(r e l)$ & G_Grimme (rel) \\
\hline $\mathrm{R}$ & $99.5 \%$ & $99.4 \%$ & $98.1 \%$ & $98.9 \%$ \\
\hline i.bp1 & 3.7 & 3.6 & 3.0 & 3.3 \\
\hline i.bp2 & 0.4 & 0.4 & 0.5 & 0.4 \\
\hline i.bp 6 & 3.6 & 3.5 & 2.7 & 3.1 \\
\hline i.bp 7 & 1.3 & 1.3 & 0.7 & 1.0 \\
\hline ii.BP1 & 3.0 & 3.0 & 2.6 & 2.8 \\
\hline ii.BP2 & 0.0 & 0.0 & 0.0 & 0.0 \\
\hline iii.BP1 & 4.2 & 4.1 & 3.4 & 3.7 \\
\hline iii.BP2 & 1.7 & 1.8 & 1.7 & 1.8 \\
\hline iii.BP3 & 10.1 & 10.0 & 9.1 & 9.5 \\
\hline iii.BP4 & 9.3 & 9.2 & 8.3 & 8.7 \\
\hline iii.BP5 & 6.5 & 6.3 & 5.8 & 6.0 \\
\hline \multicolumn{5}{|l|}{ S } \\
\hline i.bp1 & 3.4 & 3.2 & 2.8 & 3.0 \\
\hline i.bp2 & 2.5 & 2.4 & 2.1 & 2.2 \\
\hline i.bp3 & 3.8 & 4.0 & 4.1 & 4.1 \\
\hline i.bp6 & 3.5 & 3.4 & 2.8 & 3.1 \\
\hline i.bp 7 & 2.8 & 2.7 & 2.1 & 2.4 \\
\hline i.bp8 & 3.4 & 3.5 & 3.5 & 3.5 \\
\hline ii.BP1 & 2.9 & 2.9 & 2.5 & 2.7 \\
\hline ii.BP2 & 2.1 & 2.0 & 1.9 & 1.9 \\
\hline iii.BP1 & 3.9 & 3.8 & 3.4 & 3.6 \\
\hline iii.BP2 & 3.2 & 3.2 & 2.8 & 3.0 \\
\hline iii.BP3 & 4.1 & 4.1 & 4.1 & 4.1 \\
\hline iii.BP4 & 9.1 & 8.9 & 8.2 & 8.5 \\
\hline iii.BP5 & 9.7 & 9.6 & 8.7 & 9.1 \\
\hline
\end{tabular}


Absolute energies (E), enthalpies (H), RRHO free energies (G), and quasi-RRHO free energies (Grimme G), in hartrees, along with the corresponding relative values in $\mathrm{kcal} / \mathrm{mol}$ and predicted ee's, for catalyst $2 j$

$\begin{array}{lcccc}\text { Geometry } & E & \text { H } & \text { G } & \text { G_Grimme } \\ \text { R } & 90.5 \% & 72.1 \% & -5.2 \% & 30.6 \% \\ \text { iii.BP1 } & -3240.735875 & -3240.076297 & -3240.13766 & -3240.133528 \\ \text { iii.BP2 } & -3240.739297 & -3240.079467 & -3240.140383 & -3240.136346 \\ \text { iii.BP3 } & -3240.725592 & -3240.066095 & -3240.127362 & -3240.1232 \\ \text { iii.BP4 } & -3240.728392 & -3240.068839 & -3240.130585 & -3240.126271 \\ \text { iii.BP5 } & -3240.729554 & -3240.070326 & -3240.132444 & -3240.12792 \\ \text { S } & & & & \\ \text { i.bp2 } & -3240.730972 & -3240.071767 & -3240.134193 & -3240.129524 \\ \text { iii.BP1 } & -3240.737429 & -3240.078336 & -3240.140446 & -3240.135953 \\ \text { iii.BP2 } & -3240.734724 & -3240.075516 & -3240.137799 & -3240.133217 \\ \text { iii.BP3 } & -3240.734881 & -3240.075202 & -3240.136302 & -3240.132252 \\ \text { iii.BP4 } & -3240.727133 & -3240.067709 & -3240.129343 & -3240.125107 \\ \text { iii.BP5 } & -3240.726835 & -3240.067549 & -3240.129497 & -3240.125099\end{array}$

\begin{tabular}{|c|c|c|c|c|}
\hline Geometry & E & $\mathrm{H}(r \in l)$ & $G(r e l)$ & G_Grimme (rel) \\
\hline R & $90.5 \%$ & $72.1 \%$ & $-5.2 \%$ & $30.6 \%$ \\
\hline iii.BP1 & 2.1 & 2.0 & 1.7 & 1.8 \\
\hline iii.BP2 & 0.0 & 0.0 & 0.0 & 0.0 \\
\hline iii.BP3 & 8.6 & 8.4 & 8.2 & 8.2 \\
\hline iii.BP4 & 6.8 & 6.7 & 6.1 & 6.3 \\
\hline iii.BP5 & 6.1 & 5.7 & 5.0 & 5.3 \\
\hline & & & & \\
\hline i.bp2 & 5.2 & 4.8 & 3.9 & 4.3 \\
\hline iii.BP1 & 1.2 & 0.7 & 0.0 & 0.2 \\
\hline iii.BP2 & 2.9 & 2.5 & 1.6 & 2.0 \\
\hline ii.BP3 & 2.8 & 2.7 & 2.6 & 2.6 \\
\hline iii.BP4 & 7.6 & 7.4 & 6.9 & 7.1 \\
\hline ii.BP5 & 7.8 & 7.5 & 6.8 & 7.1 \\
\hline
\end{tabular}


B97-D/TZV(2d,2P) Optimized Cartesian Coordinates available in standard XYZ format as SI_coords.xyz 